\title{
MAGNETIC OSCILLATIONS IN A MODEL OF GRAPHENE
}

\author{
SIMON BECKER AND MACIEJ ZWORSKI
}

\begin{abstract}
We consider a quantum graph as a model of graphene in constant magnetic field and describe the density of states in terms of relativistic Landau levels satisfying a Bohr-Sommerfeld quantization condition. That provides semiclassical corrections (with the magnetic flux as the semiclassical parameter) in the study of magnetic oscillations.
\end{abstract}

\section{Introduction AND STATEMENT OF RESUlts}

The purpose of this paper is to describe the density of states for a model of graphene in constant magnetic field and to relate it to the Shubnikov-de Haas and de Haas-van Alphen effects.

We use a quantum graph model introduced by Kuchment-Post [KP07] with the magnetic field formalism coming from Brüning-Geyler-Pankrashin [BGP07]. Quantum graphs help to investigate spectral properties of complex systems: the complexity is captured by the graph but analytic aspects remain one dimensional and hence relatively simple. In particular, existence of Dirac points in the Bloch-Floquet dispersion relation - see $\S 3.3$ - follows from a straightforward computation. This should be compared with the subtle study by Fefferman-Weinstein [FW12] which starts with periodic Schödinger operators on $\mathbb{R}^{2}$.

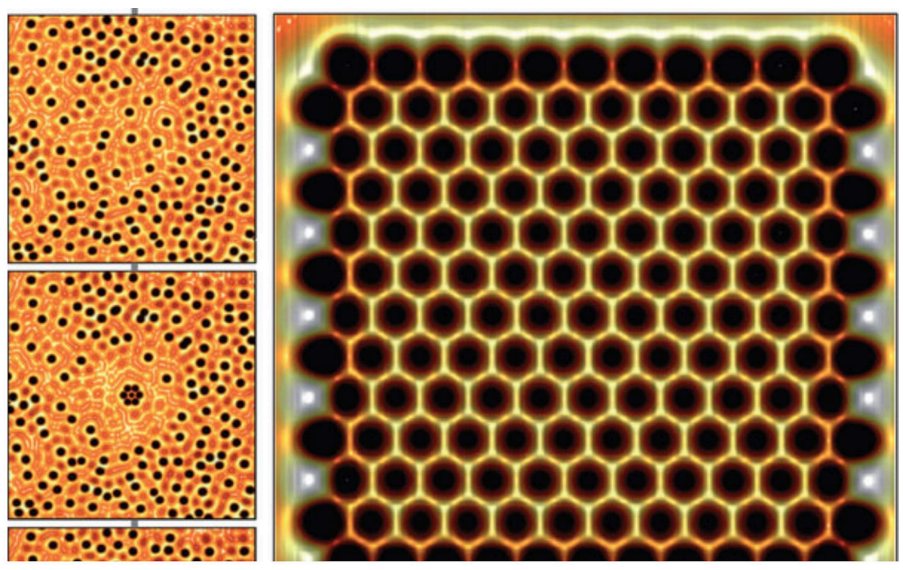

Figure 1. A molecular graphene $\left[G^{*} 12\right]$ in which the $\mathrm{CO}$ molecules confine the Bloch electron to a one dimensional hexagonal structure. 


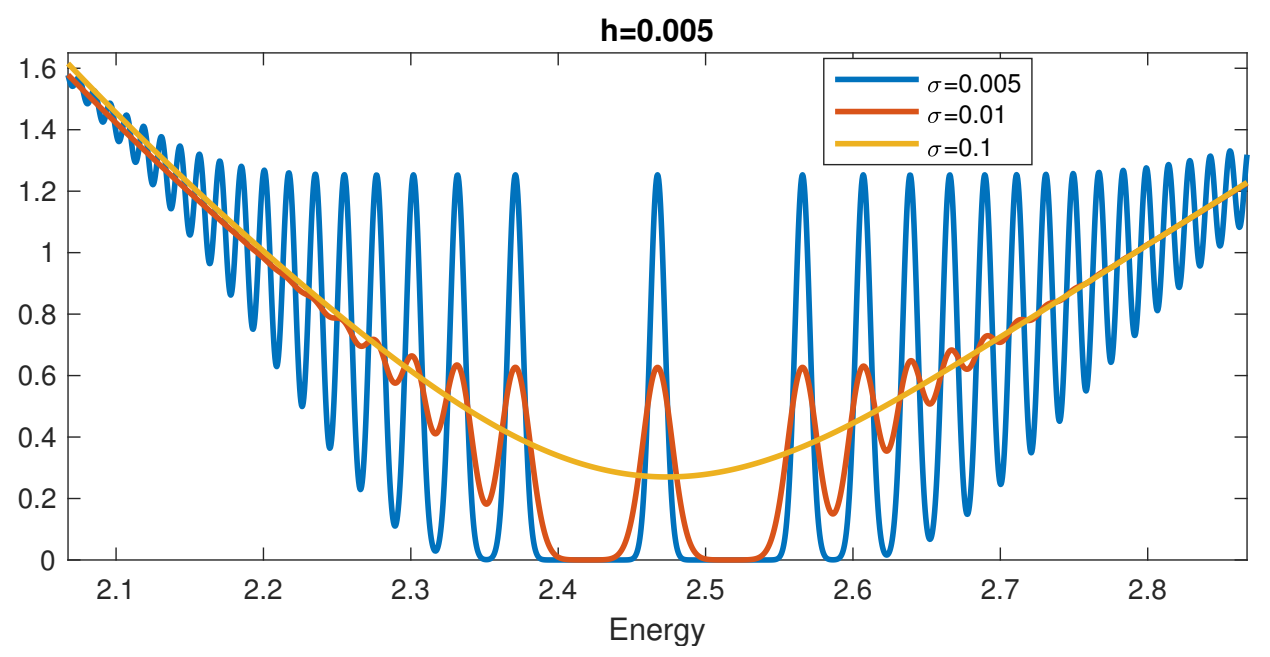

Figure 2. Graphs of smoothed-out density of states $\mu \mapsto \widetilde{\rho}_{B}(\exp ((\bullet-$ $\left.\mu)^{2} / 2 \sigma^{2}\right) / \sqrt{2 \pi} \sigma$ ) for $h=0.005$ and for different values of $\sigma$ (using an approximation (7.4)). When $\sigma$ is large the function $x \mapsto \exp ((x-$ $\left.\left.\mu)^{2} / 2 \sigma^{2}\right) / \sqrt{2 \pi} \sigma\right)$ is uniformly smooth and we see no oscillations as predicted by (1.4). Figure 9 compares these graphs with the ones based on the "perfect cone" approximation (1.2); see also Figure 7 for the density of states of the zero magnetic field.

One experimental setting for which quantum graphs could be a reasonable model is molecular graphene studied by the Manoharan group [ $\left.\mathrm{G}^{*} 12\right]$ - see also [ $\left.\mathrm{P}^{*} 13\right]$ for a general discussion. In that case $\mathrm{CO}$ molecules placed on a copper plate confine the electrons to a one dimensional hexagonal structure - see Figure 1.

The ideas behind rigorous study of the density of states and of magnetic oscillations come from the works of Helffer-Sjöstrand [HS88],[HS89],[HS90a],[HS90b],[Sj89] (to which we refer for background and additional references). However, the simplicity of our model allows us to give an essentially self-contained presentation.

The main object of our study is the density of state (DOS) for a magnetic Hamiltonian, $H_{B}$, on a hexagonal quantum graph - see (3.4). The DOS is defined as a non-negative distribution $\rho_{B} \in \mathscr{D}^{\prime}(\mathbb{R})$ (that is, a measure) produced by a renormalized trace: for $f \in C_{\mathrm{c}}(\mathbb{R})$,

$$
\rho_{B}(f)=\tilde{\operatorname{tr}}\left(f\left(H^{B}\right)\right):=\lim _{R \rightarrow \infty} \frac{\operatorname{tr} \mathbb{1}_{B(R)} f\left(H_{B}\right)}{\operatorname{vol}(B(R))}, \quad B(R):=\left\{x \in \mathbb{R}^{2}:|x|<R\right\}
$$

see Definition 4.6. 
Our desription of the density of states comes close to formal expressions for the density of states $\rho_{B}$ given in the physics literature,

$$
\begin{gathered}
\rho_{B}(E)=\frac{B}{\pi} \sum_{n \in \mathbb{Z}} \delta\left(E-E_{n}\right), \quad E_{n}:=\operatorname{sign}(n) v_{F} \sqrt{|n| B}, \\
v_{F}=\text { Fermi velocity }, \quad B=\text { strength of the magnetic field, }
\end{gathered}
$$

see for instance [CU08, (42)] or [SGB04, (4.2)]. The energies $E_{n}$ are the (approximate) relativistic Landau levels.

Theorem 1 gives the following rigorous version of (1.2). It is convenient to consider a semiclassical parameter given by the magnetic flux through a cell of the hexagonal lattice, see Figure 4:

$$
h:=\frac{3 \sqrt{3}}{2} B=\left|b_{1} \wedge b_{2}\right| B .
$$

Then, if $I$ is a neighbourhood of a Dirac energy (see $\S 3.3$ ) and $f \in C_{\mathrm{c}}^{\alpha}(I), \alpha>0$,

$$
\rho_{B}(f)=\frac{h}{\pi\left|b_{1} \wedge b_{2}\right|} \sum_{n \in \mathbb{Z}} f\left(z_{n}(h)\right)+\mathcal{O}\left(\|f\|_{C^{\alpha}} h^{\infty}\right), \quad h \rightarrow 0,
$$

where $z_{n}(h)$ satisfy natural quantization conditions (5.6) and (6.12). They are approximately given by $z_{D}+E_{n}$ with $E_{n}$ 's in (1.2), where $z_{D}$ is the Dirac energy. This simple asymptotic formula should be contrasted with the complicated structure of the spectrum of $H^{B}$ - see the analysis by Becker-Han-Jitomirskaya [BHJ17].

The importance of considering functions which are not smooth is their appearance in condensed matter calculations - see $\S 7$. Oscillations as functions of $1 / B$ are not seen for smooth functions in view of Theorem 2 :

$$
\rho_{B}(f) \sim \sum_{j=0}^{\infty} A_{j}(f) h^{j}, \quad A_{0}(f)=\rho_{0}(f), \quad h \rightarrow 0, \quad f \in C_{\mathrm{c}}^{\infty}(I) .
$$

Roughly speaking, this expansion follows from the expansion of the Riemann sum given by (1.3) - see [HS90b]. Here the proof follows [DS99, Chapter 8].

Many physical quantities are computed using DOS, in particular grand-canonical potentials and magnetizations at temperature $T=1 / \beta$ localized to an energy interval using a function $\eta$ :

$$
\begin{gathered}
\Omega_{\beta}(\mu, h):=\rho_{B}\left(\eta(\bullet) f_{\beta}(\mu-\bullet)\right), \quad f_{\beta}(x):=-\beta^{-1} \log \left(e^{\beta x}+1\right), \\
M_{\beta}(\mu, h):=-\left|b_{1} \wedge b_{2}\right| \frac{\partial}{\partial h} \Omega_{\beta}(\mu, h) .
\end{gathered}
$$

For $\beta=\infty$ we take $f_{\infty}=x_{+}$which is a Lipschitz function. Hence (1.3) applies and for $\beta>h^{-M_{0}}$ one could also obtain expansions for $M_{\beta}$ - see $\S 7.2$. We take a simpler approach and calculate a semiclassical approximation, $m_{\infty}(\mu, h)$, to magnetization see (7.10) and compare it to (almost) exact spectral calculations - see $§ \S 7.3,7.4$. The 


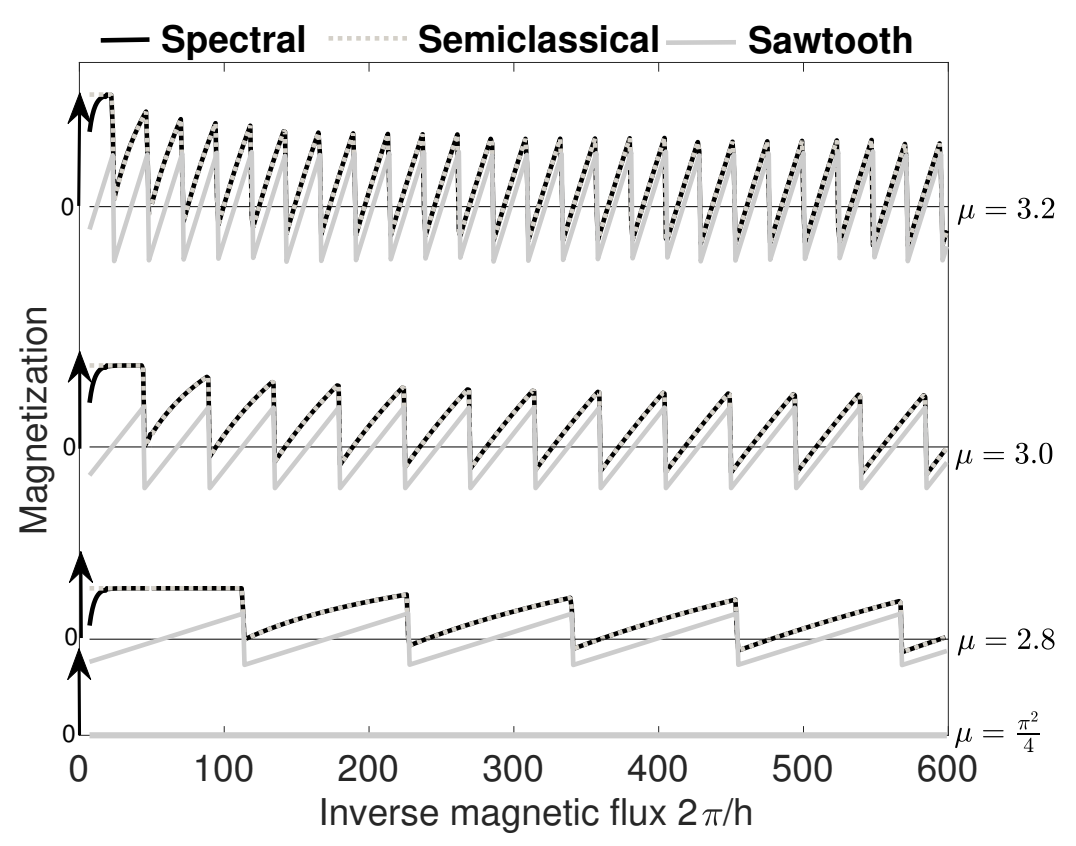

Figure 3. Plots of different approximations of the magnetization localized to the upper cone: the spectral one using $M_{\infty}$ defined in (1.5) (see $\S 7.3)$, the semiclassical approximation $m_{\infty}$ given in (7.10) and the sawtooth approximation (1.6). The agreement of $M_{\infty}$ and $m_{\infty}$ is remarkable even for relatively large values $h$. The sawtooth approximation gives the correct oscillations but with amplitude errors $\mathcal{O}(\sqrt{h})$.

agreement between $M_{\infty}$ computed spectrally and the approximation $m_{\infty}$ is remarkable already at fairly high values of the magnetic field. In Theorem 3 we derive a simple "sawtooth" approximation for $m_{\infty}$ confirming approximations seen in the physics literature [SGB04],[CM01]:

$$
\begin{gathered}
m_{\infty}(\mu, h)=\frac{1}{\pi} \sigma\left(\frac{g(\mu)}{h}\right) \frac{g(\mu)}{g^{\prime}(\mu)}+\mathcal{O}\left(h^{\frac{1}{2}}\right), \\
\sigma(y):=y-[y]-\frac{1}{2} .
\end{gathered}
$$

The function $g$ comes from the dispersion relation for the quantum graph model of graphene [KP07] (see $\S 3.3)$ :

$$
g(\mu):=\frac{1}{4 \pi} \int_{\gamma_{\Delta(\mu)^{2}}} \xi d x, \quad \gamma_{\omega}=\left\{(x, \xi) \in \mathbb{R}^{2} / 2 \pi \mathbb{Z}^{2}: \frac{\left|1+e^{i x}+e^{i \xi}\right|^{2}}{9}=\omega\right\},
$$

where $\Delta(\mu)$ is the Floquet discriminant of the potential on the edges (and is equal to $\cos \sqrt{\lambda}$ for the zero potential). The Dirac energy, $z_{D}$, for a given band is determined by $z_{D}=\left.\Delta\right|_{B_{k}} ^{-1}(0)$. 
Notation. We write $f_{\alpha}=\mathcal{O}_{\alpha}(g)_{H}$ for $\|f\|_{H} \leq C_{\alpha} g$, that is we have a bound with constants depending on $\alpha$. In particular, $f=\mathcal{O}\left(h^{\infty}\right)_{H}$ means that for any $N$ there exists $C_{N}$ such that $\|f\|_{H} \leq C_{N} h^{N}$. We denote $\langle x\rangle:=\sqrt{1+|x|^{2}}$.

Acknowledgements. We gratefully acknowledge support by the UK Engineering and Physical Sciences Research Council (EPSRC) grant EP/L016516/1 for the University of Cambridge Centre for Doctoral Training, the Cambridge Centre for Analysis (SB), by the National Science Foundation under the grant DMS-1500852 and by the Simons Foundation (MZ). We would also like to thank Nicolas Burq for useful discussions, Semyon Dyatlov for help with MATLAB coding and insightful comments and Hari Manoharan for introducing us to molecular graphene and for allowing us to use Figures 1 and $7(\mathrm{~B})$.

\section{HeXagonal quantum Graphs}

Quantum graphs provide a simple model for a graphene-like structure in which many features can be rigorously derived with minimal technical effort. Hence we consider a hexagonal graph, $\Lambda$, with Schrödinger operators defined on each edge [KP07]. The graph $\Lambda$ is obtained by translating its fundamental cell $W_{\Lambda}$, consisting of vertices

$$
r_{0}:=(0,0), \quad r_{1}:=\left(\frac{1}{2}, \frac{\sqrt{3}}{2}\right)
$$

and edges

$$
\begin{aligned}
& f:=\operatorname{conv}\left(\left\{r_{0}, r_{1}\right\}\right) \backslash\left\{r_{0}, r_{1}\right\}, \\
& g:=\operatorname{conv}\left(\left\{r_{0},(-1,0)\right\}\right) \backslash\left\{r_{0},(-1,0)\right\}, \\
& h:=\operatorname{conv}\left(\left\{r_{0},-r_{1}\right\}\right) \backslash\left\{r_{0},-r_{1}\right\},
\end{aligned}
$$

along the basis vectors of the lattice. The basis vectors are

$$
b_{1}:=\left(\frac{3}{2}, \frac{\sqrt{3}}{2}\right) \text { and } b_{2}:=(0, \sqrt{3})
$$

and so the hexagonal graph $\Lambda \subset \mathbb{R}^{2}$ is given by the range of a $\mathbb{Z}^{2}$-action on the fundamental domain $W_{\Lambda}$

$$
\Lambda:=\left\{x \in \mathbb{R}^{2} ; x=\gamma_{1} b_{1}+\gamma_{2} b_{2}+[x] \text { for } \gamma \in \mathbb{Z}^{2} \text { and }[x] \in W_{\Lambda}\right\} .
$$

The set of edges of $\Lambda$ is denoted by $\mathcal{E}=\mathcal{E}(\Lambda)$, the set of vertices by $\mathcal{V}=\mathcal{V}(\Lambda)$ and the set of edges adjacent to a given vertex $v \in \mathcal{V}$ by $\mathcal{E}_{v}$. We drop $\Lambda$ in the notation if no confusion is likely to arise.

When we say that $u \in C(\Lambda)$ we mean that $u$ is a continuous function on $\Lambda$, a closed subset of $\mathbb{R}^{2}$ - see (2.4).

For any edge $e \in \mathcal{E}$ we denote by $[e] \in \mathcal{E}\left(W_{\Lambda}\right)$ the unique edge (thought of as a vector in $\mathbb{R}^{2}$ ) for which there is $\gamma \in \mathbb{Z}^{2}$ such that $e=\gamma_{1} b_{1}+\gamma_{2} b_{2}+[e]$. We impose a 


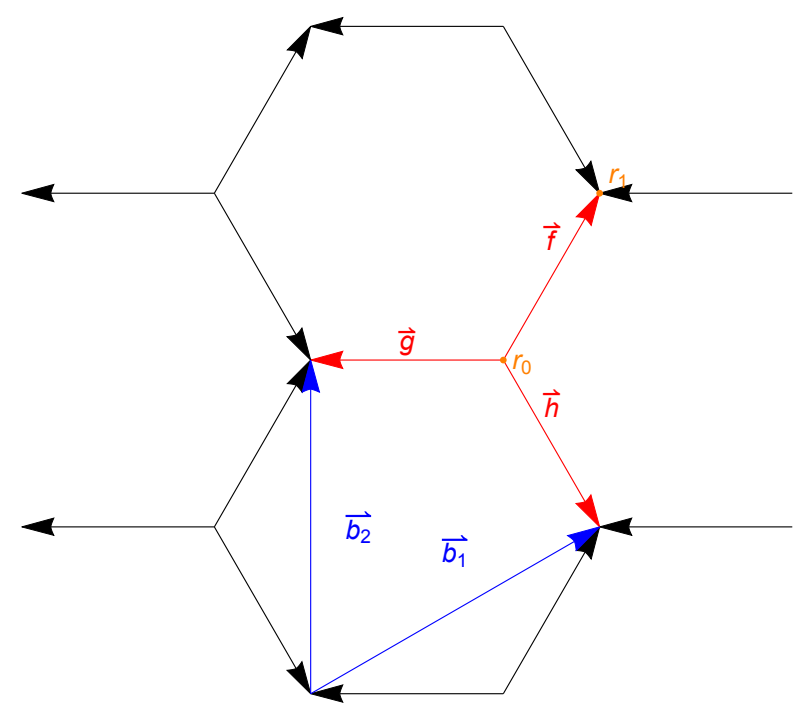

Figure 4. The fundamental cell and lattice basis vectors of $\Lambda$.

global orientation on the graph by orienting the edges in terms of initial and terminal maps

$$
i: \mathcal{E} \rightarrow \mathcal{V}, \quad t: \mathcal{E} \rightarrow \mathcal{V}
$$

where $i$ and $t$ map edges to their initial and terminal ends. It suffices to specify the orientation on the fundamental domain

$$
i(f)=i(g)=i(h)=r_{0}, \quad t(f)=r_{1}, t(g)=r_{1}-b_{1}, \quad t(h)=r_{1}-b_{2} .
$$

For arbitrary $e \in \mathcal{E}$, we then extend those maps by

$$
i(e):=\gamma_{1} b_{1}+\gamma_{2} b_{2}+i([e]) \text { and } t(e):=\gamma_{1} b_{1}+\gamma_{2} b_{2}+t([e]) .
$$

In the case of our special graph with orientations showed in Figure 4 a given vertex is either initial or terminal and hence we wrote

$$
\mathcal{V}=\mathcal{V}^{i} \sqcup \mathcal{V}^{t}, \quad \mathcal{V}^{\bullet}:=\{v: v=\bullet(e) \text { for some } e \in \mathcal{E}\}, \bullet=i, t .
$$

The fundamental domain of the dual lattice can be identified, because the lattice is spanned by a $\mathbb{Z}^{2}$-action, with the dual 2-torus

$$
\mathbb{T}_{*}^{2}:=\mathbb{R}^{2} /(2 \pi \mathbb{Z})^{2}
$$

We assume every edge $e \in \mathcal{E}$ is of length one and has a standard chart

$$
\kappa_{e}: e \rightarrow(0,1), \quad \kappa_{e}(t i(e)+(1-t) t(e))=t .
$$

Thus, for $n \in \mathbb{N}_{0}$, the Sobolev space $H^{n}(\mathcal{E})$ on $\Lambda$ is given by the Hilbert space direct sum

$$
H^{n}(\mathcal{E}):=\bigoplus_{e \in \mathcal{E}} H^{n}(e)
$$


On edges $e \in \mathcal{E}$ we define the maximal Schrödinger operator

$$
H_{e}: H^{2}(e) \subset L^{2}(e) \rightarrow L^{2}(e), \quad H_{e} \psi_{e}:=-\psi_{e}^{\prime \prime}+V \psi_{e}
$$

with potential $V \in L^{2}((0,1)) \simeq L^{2}(e)$ which is the same on every edge and even with respect to the edge's centre.

2.1. Relation to Hill operators. Using the potential introduced in the previous section, we define the $\mathbb{Z}$-periodic Hill potential $V_{\text {per }} \in L_{\text {loc }}^{2}(\mathbb{R})$

$$
V_{\text {per }}(x+n):=V(x), \quad n \in \mathbb{Z}, x \in(0,1) .
$$

Next, we study the associated self-adjoint Hill operator on the real line

$$
H_{\text {per }}: H^{2}(\mathbb{R}) \subset L^{2}(\mathbb{R}) \rightarrow L^{2}(\mathbb{R}) \quad H_{\text {per }} \psi:=-\psi^{\prime \prime}+V \psi .
$$

There are always two linearly independent solutions $c_{\lambda}, s_{\lambda} \in H_{\mathrm{loc}}^{2}(\mathbb{R})$ to $H_{\mathrm{per}} \psi=\lambda \psi$ satisfying

$$
c_{\lambda}(0)=1, c_{\lambda}^{\prime}(0)=0 \text { and } s_{\lambda}(0)=0, s_{\lambda}^{\prime}(0)=1 .
$$

Consider the Dirichlet operator on $(0,1)$

$$
\Lambda_{(0,1)}^{D}: H_{0}^{1}(0,1) \cap H^{2}(0,1) \subset L^{2}(0,1) \rightarrow L^{2}(0,1) \quad \Lambda_{(0,1)}^{D} \psi=-\psi^{\prime \prime}+V_{\text {per }} \psi .
$$

Any function $\psi_{\lambda} \in H^{2}(0,1)$ satisfying $-\psi_{\lambda}^{\prime \prime}+V_{\text {per }} \psi_{\lambda}=\lambda \psi_{\lambda}$ with $\lambda \notin \operatorname{Spec}\left(\Lambda_{(0,1)}^{D}\right)$ (that is with $s_{\lambda}(1) \neq 0$ ) can be written as a linear combination of $s_{\lambda}, c_{\lambda}$ :

$$
\psi_{\lambda}(t)=\frac{\psi_{\lambda}(1)-\psi_{\lambda}(0) c_{\lambda}(1)}{s_{\lambda}(1)} s_{\lambda}(t)+\psi_{\lambda}(0) c_{\lambda}(t) .
$$

For $\lambda \notin \operatorname{Spec}\left(\Lambda_{(0,1)}^{D}\right)$, we define the Dirichlet-to-Neumann map

$$
m(\lambda):=\frac{1}{s_{\lambda}(1)}\left(\begin{array}{cc}
-c_{\lambda}(1) & 1 \\
1 & -s_{\lambda}^{\prime}(1)
\end{array}\right), \quad\left(\begin{array}{r}
\psi_{\lambda}^{\prime}(0) \\
-\psi_{\lambda}^{\prime}(1)
\end{array}\right)=m(\lambda)\left(\begin{array}{l}
\psi_{\lambda}(0) \\
\psi_{\lambda}(1)
\end{array}\right) .
$$

Remark 1. Since $V_{\text {per }}$ is assumed to be symmetric with respect to $\frac{1}{2}$ on the interval $(0,1), c_{\lambda}(1)=s_{\lambda}^{\prime}(1)$. The Dirichlet eigenfunctions are consequently either even or odd with respect to $\frac{1}{2}$.

The monodromy matrix associated with $H_{\text {per }}$ is the matrix valued entire function of $\lambda$ :

$$
\mathcal{M}(\lambda):=\left(\begin{array}{ll}
c_{\lambda}(1) & s_{\lambda}(1) \\
c_{\lambda}^{\prime}(1) & s_{\lambda}^{\prime}(1)
\end{array}\right)
$$

Its normalized trace

$$
\Delta(\lambda):=\frac{\operatorname{tr}(\mathcal{M}(\lambda))}{2}
$$

is called the Floquet discriminant. In the case when $V \equiv 0$ we have

$$
\Delta(\lambda)=\cos \sqrt{\lambda}
$$

and this will serve as an example throughout the paper. 
The spectrum of the Hill operator, $H_{\text {per }}$ is purely absolutely continuous spectrum and is given by

$$
\begin{gathered}
\operatorname{Spec}\left(H_{\text {per }}\right)=\{\lambda \in \mathbb{R}:|\Delta(\lambda)| \leq 1\}=\bigcup_{n=1}^{\infty} B_{n} \\
B_{n}:=\left[\alpha_{n}, \beta_{n}\right], \quad \beta_{n} \leq \alpha_{n+1},\left.\quad \Delta^{\prime}\right|_{\operatorname{int}\left(B_{n}\right)} \neq 0,
\end{gathered}
$$

see $[\mathrm{RS} 78, \S \mathrm{XIII}]$.

\section{Magnetic Hamiltonians on quantum GRaphs}

The vector potential $\mathbf{A}$ is a one form on $\mathbb{R}^{2}$ and the magnetic field is given by $\mathbf{B}=d \mathbf{A}$. For a homogeneous magnetic field

$$
\mathbf{B}:=B d x_{1} \wedge d x_{2}
$$

we can choose a symmetric gauge, that is $\mathbf{A}$ given as follows:

$$
\mathbf{B}=d \mathbf{A}, \quad \mathbf{A}=\frac{1}{2} B\left(-x_{2} d x_{1}+x_{1} d x_{2}\right) .
$$

The scalar vector potential $A_{e} \in C^{\infty}(e)$ along edges $e \in \mathcal{E}$ is obtained by evaluating the form on the graph along the vector field generated by the respective edge $[e]$ :

$$
\begin{aligned}
A_{e}(t) & :=\mathbf{A}(i(e)+t[e])\left([e]_{1} \partial_{1}+[e]_{2} \partial_{2}\right) \\
& =\mathbf{A}(i(e))\left([e]_{1} \partial_{1}+[e]_{2} \partial_{2}\right)+\underbrace{t A([e])\left([e]_{1} \partial_{1}+[e]_{2} \partial_{2}\right)}_{=0} \\
& =\mathbf{A}(i(e))\left([e]_{1} \partial_{1}+[e]_{2} \partial_{2}\right)
\end{aligned}
$$

which is constant along any single edge.

In terms of the magnetic differential operator $\left(D^{B} \psi\right)_{e}:=-i \psi_{e}^{\prime}-A_{e} \psi_{e}$, the Schrödinger operator modeling graphene in a magnetic field becomes

$$
H^{B}: D\left(H^{B}\right) \subset L^{2}(\mathcal{E}) \rightarrow L^{2}(\mathcal{E}), \quad\left(H^{B} \psi\right)_{e}:=\left(D^{B} D^{B} \psi\right)_{e}+V \psi_{e},
$$

where $D\left(H^{B}\right)$ is defined as the set of $\psi \in H^{2}(\mathcal{E})$ satisfying

$$
\psi_{e_{1}}(v)=\psi_{e_{2}}(v), e_{1}, e_{2} \in \mathcal{E}_{v}, \quad \sum_{e \in \mathcal{E}_{v}}\left(D^{B} \psi\right)_{e}(v)=0 .
$$

Remark 2. The Hamiltonian $H^{B}$ for any magnetic field with constant flux per hexagon is unitarily equivalent to the setting of a constant magnetic field with the same flux per hexagon.

The unitary Peierls' substitution is the multiplication operator

$$
P: L^{2}(\mathcal{E}) \rightarrow L^{2}(\mathcal{E}), \quad \psi_{e}(t) \mapsto e^{i A_{e} t} \psi_{e}(t), \quad t \in(0,1)
$$

The operator $P$ transforms $H^{B}$ into

$$
\Lambda^{B}:=P^{-1} H^{B} P, \quad\left(\Lambda^{B} \psi\right)_{e}=-\psi_{e}^{\prime \prime}+V \psi_{e} .
$$


The domain of $\Lambda^{B}$ consists of $\psi \in H^{2}(\mathcal{E})$ such that, in the notation of (2.6),

$$
\begin{aligned}
& v \in \mathcal{V}^{i} \Longrightarrow \psi_{e_{1}}(v)=\psi_{e_{2}}(v), \quad e_{1}, e_{2} \in \mathcal{E}_{v}, \quad \sum_{e \in \mathcal{E}_{v}} \psi_{e}^{\prime}(v)=0, \\
& v \in \mathcal{V}^{t} \Longrightarrow e^{i A_{e_{1}}} \psi_{e_{1}}(v)=e^{i A_{e_{2}}} \psi_{e_{2}}(v), \quad e_{1}, e_{2} \in \mathcal{E}_{v}, \quad \sum_{e \in \mathcal{E}_{v}} e^{i A_{e}} \psi_{e}^{\prime}(v)=0 .
\end{aligned}
$$

Thus, the problem reduces to the study of non-magnetic Schrödinger operators with the magnetic field moved into the boundary conditions. We note that the magnetic Dirichlet operator,

$$
H^{D}: \bigoplus_{e \in \mathcal{E}(\Lambda)}\left(H_{0}^{1}(e) \cap H^{2}(e)\right) \rightarrow L^{2}(\mathcal{E}), \quad\left(H^{D} \psi\right)_{e}:=\left(D^{B} D^{B} \psi\right)_{e}+V_{e} \psi_{e}
$$

is (using Peierls' substitution (3.5)) unitarily equivalent to the Dirichlet operator without magnetic field

$$
\Lambda^{D}:=\bigoplus_{e \in \mathcal{E}(\Lambda)} \Lambda_{e}^{D}=P^{-1} H^{D} P
$$

where $\Lambda_{e}^{D}$ is the Dirichlet realization of $-\partial_{t}^{2}+V_{e}$ on $e$. Thus, the spectrum of the Dirichlet operator does not change under magnetic perturbations.

3.1. Effective Hamiltonian. We now follow Pankrashin [Pa06] and Brüning-GeylerPankrashin[BGP07] and use the Krein resolvent formula to reduce the operator $\Lambda^{B}$ into a term containing only parts of the Dirichlet spectrum and an effective operator that will be further investigated afterwards. We will find that the contribution of Dirichlet eigenvalues to the spectrum of $H^{B}$ is fully explicit and thus we will be left with an effective operator which will be used to describe the density of states.

We define

$$
\mathbf{H}: D(\mathbf{H}) \subset L^{2}(\mathcal{E}) \rightarrow L^{2}(\mathcal{E}), \quad(\mathbf{H} \psi)_{e}:=\left(D^{B} D^{B} \psi\right)_{e}+V_{e} \psi_{e}
$$

where $D(\mathbf{H})$ consists of $\psi \in H^{2}(\mathcal{E})$ satifying (using notation of (2.6))

$$
\begin{aligned}
& v \in \mathcal{V}^{i} \Longrightarrow \psi_{e_{1}}(v)=\psi_{e_{2}}(v), \quad e_{1}, e_{2} \in \mathcal{E}_{v}, \\
& v \in \mathcal{V}^{t} \Longrightarrow e^{i A_{e_{1}}} \psi_{e_{1}}\left(t\left(e_{1}\right)\right)=e^{i A_{e_{2}}} \psi_{e_{2}}\left(t\left(e_{2}\right)\right), \quad e_{1}, e_{2} \in \mathcal{E}_{v} .
\end{aligned}
$$

With this domain $\mathbf{H}$ is a closed operator.

Then, we consider the map $\pi: D(\mathbf{H}) \rightarrow \ell^{2}(\mathcal{V})$ defined by

$$
\pi(\psi)(v):=\left\{\begin{array}{cll}
\psi_{e}(v), & v \in \mathcal{V}^{i}, & e \in \mathcal{E}_{v} \\
e^{i A_{e}} \psi_{e}(v), & v \in \mathcal{V}^{t}, & e \in \mathcal{E}_{v}
\end{array}\right.
$$

The operator $\pi$ is well defined because of (3.9) and is an isomorphism from $\operatorname{ker}(\mathbf{H}-\lambda)$ onto $\ell^{2}(\mathcal{V})$ for any $\lambda \notin \operatorname{Spec}\left(\Lambda^{D}\right)$. This leads to the definition of the gamma-field

$$
\gamma: \complement \operatorname{Spec}\left(\Lambda^{D}\right) \rightarrow \mathcal{L}\left(\ell^{2}(\mathcal{V}), D(\mathbf{H})\right), \quad \gamma(\lambda):=\left(\left.\pi\right|_{\operatorname{ker}(\mathbf{H}-\lambda)}\right)^{-1}
$$


In the notation of (2.12), the gamma-field is given by

$$
(\gamma(\lambda) z)_{e}(t)=\frac{\left(s_{\lambda}(1) c_{\lambda}(t)-s_{\lambda}(t) c_{\lambda}(1)\right) z(i(e))+e^{-i A_{e}} z(t(e)) s_{\lambda}(t)}{s_{\lambda}(1)} .
$$

Using this we can then state Krein's formula from [Pa06] and [BGP07]. For that we define

$$
M(\lambda):=s_{\lambda}(1)^{-1}\left(K_{\Lambda}-\Delta(\lambda)\right)
$$

where

$$
\left(K_{\Lambda} z\right)(v):=\frac{1}{3}\left(\sum_{e \in \mathcal{E}, i(e)=v} e^{-i A_{e}} z(t(e))+\sum_{e \in \mathcal{E}, t(e)=v} e^{i A_{e}} z(i(e))\right)
$$

defines an operator on $\ell^{2}(\mathcal{V})$ with $\left\|K_{\Lambda}\right\| \leq 1$.

Proposition 3.1 (Krein's resolvent formula). Let $\Lambda^{B}$ and $\Lambda^{D}$ be given by (3.6) and (3.8) respectively. For $\lambda \notin \operatorname{Spec}\left(\Lambda^{D}\right) \cup \operatorname{Spec}\left(\Lambda^{B}\right)$ the operator $M(\lambda)$ is invertible and satisfies

$$
\left(\Lambda^{B}-\lambda\right)^{-1}=\left(\Lambda^{D}-\lambda\right)^{-1}-\gamma(\lambda) M(\lambda)^{-1} \gamma(\bar{\lambda})^{*},
$$

where $M(\lambda)$ is given by (3.13).

As a consequence of (3.15) we see that

$$
\operatorname{Spec}\left(\Lambda^{B}\right) \backslash \operatorname{Spec}\left(\Lambda^{D}\right)=\left\{\lambda \in \complement \operatorname{Spec}\left(\Lambda^{D}\right) ; 0 \in \operatorname{Spec}(M(\lambda))\right\} .
$$

If $\lambda \notin \operatorname{Spec}\left(\Lambda^{D}\right)$ it follows that $\gamma(\lambda) \operatorname{ker}(M(\lambda))=\operatorname{ker}\left(\Lambda^{B}-\lambda\right)$. This implies that both null-spaces are of equal dimension.

Remark 3. The general theory of spectral triples gives the following formula for the derivative for $M$,

$$
\partial_{\lambda} M(\lambda)=\gamma(\bar{\lambda})^{*} \gamma(\lambda)
$$

see [Sch12, Proposition 14.5]. This will be important later.

3.2. Magnetic translations. The magnetic Schrödinger operator $H^{B}$ does not commute with standard lattice translation operators

$$
T_{\gamma} \psi(x):=\psi\left(x-\gamma_{1} b_{1}-\gamma_{2} b_{2}\right)
$$

It does however commute with modified translations which do not commute with each other in general. Those magnetic translations $T_{\gamma}^{B}: L^{2}(\mathcal{E}) \rightarrow L^{2}(\mathcal{E})$ are unitary operators defined by

$$
T_{\gamma}^{B} \psi:=u^{B}(\gamma) T_{\gamma} \psi, \quad \psi=\left(\psi_{e}\right)_{e \in \mathcal{E}} \in L^{2}(\mathcal{E}), \quad \gamma \in \mathbb{Z}^{2},
$$


To define $u^{B}$ we first consider it as $u^{B}: \mathbb{Z}^{2} \rightarrow C\left(W_{\Lambda}\right)$ where $W_{\Lambda}$ is the fundamental domain defined in (2.1) and (2.2):

$$
\begin{gathered}
u^{B}(\gamma)_{e}(s i(e)+(1-s) t(e)):=e^{i \alpha_{e}(\gamma) s}, \quad e \in W_{\Lambda}, \\
\alpha_{e}(\gamma):=A\left(\gamma_{1} b_{1}+\gamma_{2} b_{2}\right)\left([e]_{1} \partial_{1}+[e]_{2} \partial_{2}\right), \\
u^{B}(\gamma)\left(r_{0}\right):=1, \quad u^{B}(\gamma)\left(r_{1}\right):=e^{i \alpha_{f}(\gamma)} .
\end{gathered}
$$

We then extend $u^{B}$ to the graph using translations. Using (3.3) we see that

$$
\begin{aligned}
& \alpha_{f}(\gamma)=\frac{B}{2} \frac{\sqrt{3}}{2}\left(\gamma_{1}-\gamma_{2}\right)=\frac{h}{6}\left(\gamma_{1}-\gamma_{2}\right), \\
& \alpha_{g}(\gamma)=\frac{B}{2} \frac{\sqrt{3}}{2}\left(\gamma_{1}+2 \gamma_{2}\right)=\frac{h}{6}\left(\gamma_{1}+2 \gamma_{2}\right), \\
& \alpha_{h}(\gamma)=-\frac{B}{2} \frac{\sqrt{3}}{2}\left(2 \gamma_{1}+\gamma_{2}\right)=-\frac{h}{6}\left(2 \gamma_{1}+\gamma_{2}\right)
\end{aligned}
$$

where

$$
h:=\frac{3 \sqrt{3}}{2} B=B\left|b_{1} \wedge b_{2}\right|
$$

is the magnetic flux through one hexagon of the graph. For any $\gamma, \delta \in \mathbb{Z}^{2}$

$$
u^{B}(\gamma)_{[e]-\delta_{1} b_{1}-\delta_{2} b_{2}}:=e^{i \frac{h \omega(\delta, \gamma)}{2}} u^{B}(\gamma)_{[e]}
$$

where $\omega(\delta, \gamma):=\delta_{1} \gamma_{2}-\delta_{2} \gamma_{1}$ is the standard symplectic form on $\mathbb{R}^{2}$. A computation shows that $T_{\bullet}^{B}$ satisfies the commutation relation

$$
T_{\gamma}^{B} T_{\delta}^{B}=e^{i h \omega(\gamma, \delta)} T_{\delta}^{B} T_{\gamma}^{B} .
$$

It also follows that $T_{\gamma}^{B}\left(D\left(H^{B}\right)\right)=D\left(H^{B}\right)$, and that $T_{\gamma}^{B}$ are unitary operators.

Since

$$
T_{\gamma}^{B} H^{B}=H^{B} T_{\gamma}^{B}
$$

it follows that for every bounded measurable function $f: \mathbb{R} \rightarrow \mathbb{C}$

$$
T_{\gamma}^{B} f\left(H^{B}\right)=f\left(H^{B}\right) T_{\gamma}^{B} .
$$

3.3. Dirac points and band velocities. It is well-known that the energy as a function of quasimomenta for graphene has two conical cusps at energies Dirac energies:

$$
z_{D}:=\left.\Delta\right|_{B_{n}} ^{-1}(0) \quad(\text { we drop the index } n)
$$

Those cones (see Figure 5) in the energy-quasimomentum representation are referred to as Dirac cones. The name is derived from the linear energy-momentum relation for relativistic massless fermions the Dirac equation predicts.

The Hamiltonian $H^{B}$ with $B=0$ is translational invariant, that is, it commutes with translation operators $T_{\gamma}$ defined in (3.17). Using standard Floquet-Bloch theory, 


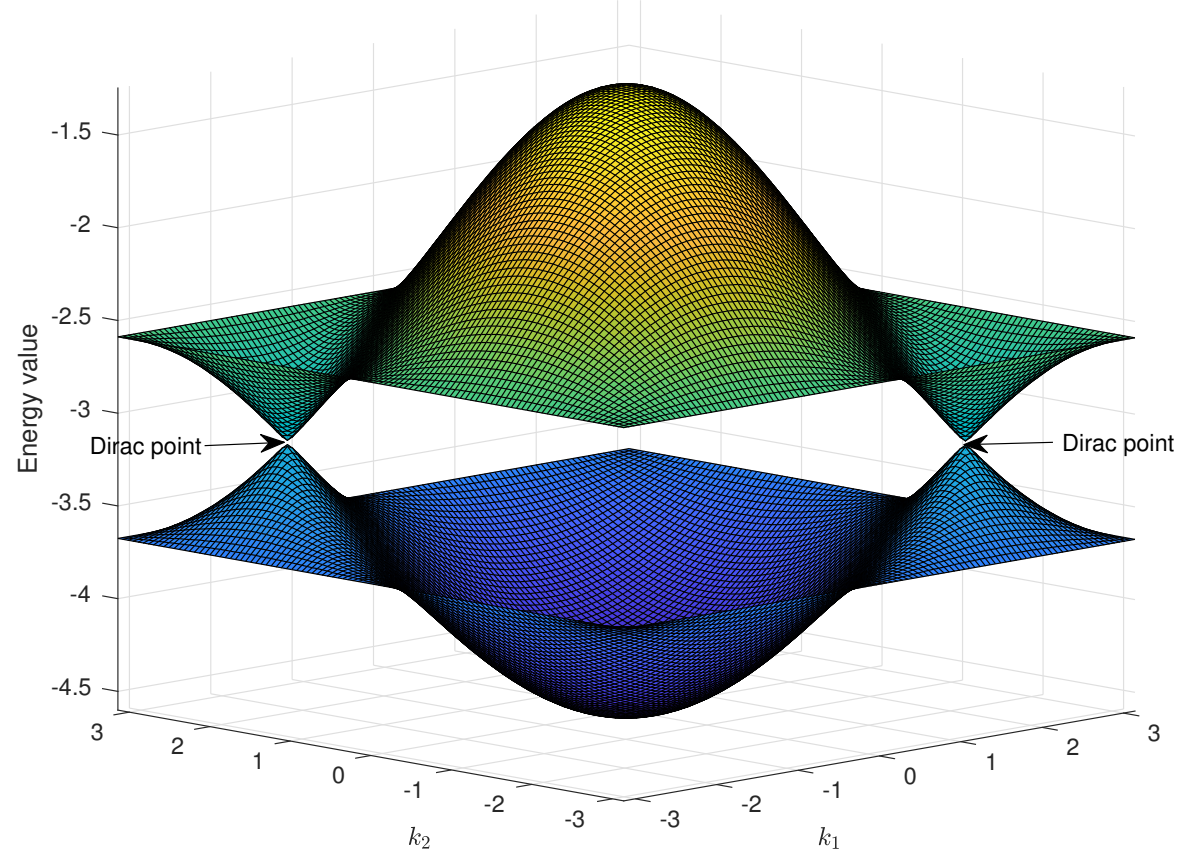

Figure 5. The first two bands of the Schrödinger operator with a Mathieu potential without magnetic perturbation showing the characteristic conical Dirac points at energy level $\approx-\pi$ where the two bands touch.

one can then diagonalize the operator $H^{B=0}$ as in Kuchment-Post [KP07] to write the spectrum for quasimomenta $\left(k_{1}, k_{2}\right) \in \mathbb{T}_{*}^{2}($ see $(2.7))$ in terms of a two-valued function

$$
\left.\mathbb{T}_{*}^{2} \ni k \mapsto \lambda^{ \pm}\right|_{B_{n}}(k):=\left.\Delta\right|_{B_{n}} ^{-1}\left( \pm \frac{\left|1+e^{i k_{1}}+e^{i k_{2}}\right|}{3}\right)
$$

on every Hill band $B_{n}$ (2.17). Expanding $\left.\lambda^{ \pm}\right|_{B_{n}}$ in polar coordinates at the Dirac points $k= \pm\left(\frac{2 \pi}{3},-\frac{2 \pi}{3}\right)$ yields the linearized energy level sets above $(+)$ and below $(-)$ the conical point

$$
\left.\lambda^{ \pm}\right|_{B_{n}}(r, \varphi):=z_{D} \pm \frac{\left.\Delta\right|_{B_{n}} ^{-1^{\prime}}(0)}{3} \sqrt{1-\frac{\sin (2 \varphi)}{2}} r+o(r)
$$

where $r$ is the distance from $k= \pm\left(\frac{2 \pi}{3},-\frac{2 \pi}{3}\right)$.

Definition 3.2 (Band velocities). The Bloch state velocity associated with quasimomenta $\left(k_{1}, k_{2}\right) \neq \pm\left(\frac{2 \pi}{3},-\frac{2 \pi}{3}\right)$ is just

$$
\left.v^{ \pm}\right|_{B_{n}}(k)=\left.\nabla \lambda^{ \pm}\right|_{B_{n}}(k)
$$

and is fully explicit using (3.26). 


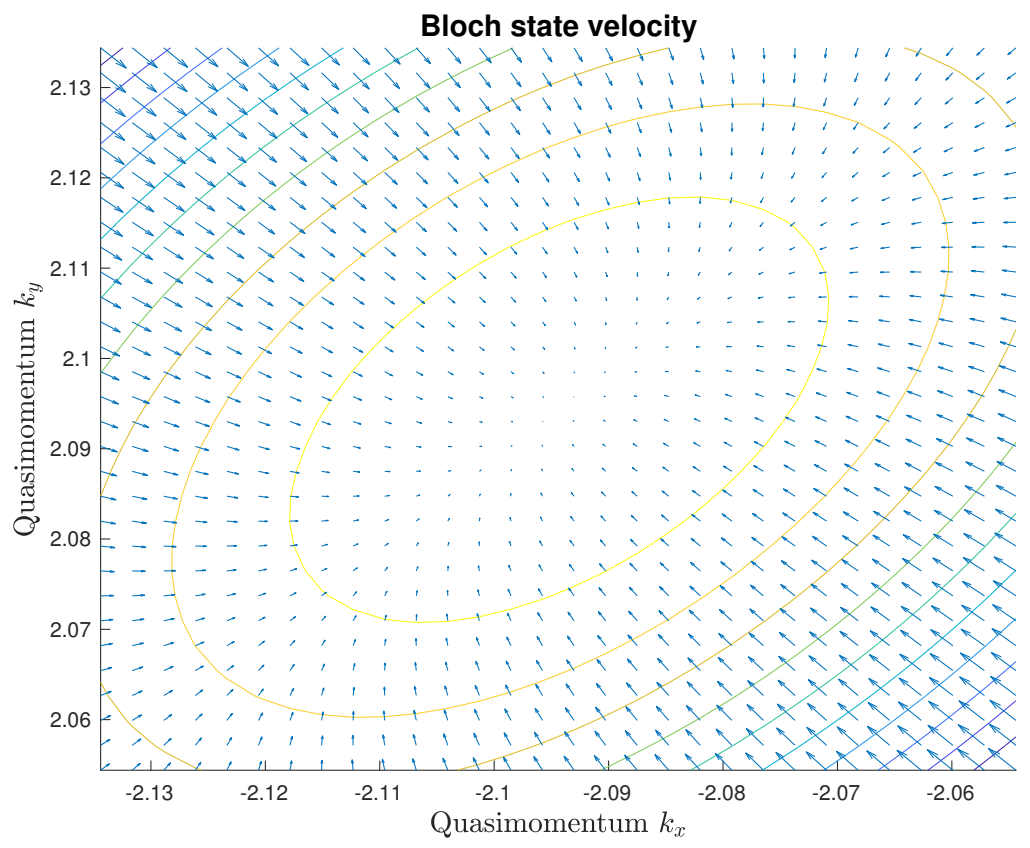

Figure 6 . The Bloch state velocity of the upper cone near the Dirac point located at $\left(k_{x}, k_{y}\right)=\frac{2 \pi}{3}(-1,1)$ for zero potential $V_{e}=0$. In particular, the Bloch state velocity is not rotationally invariant.

Remark 4. The notion of a Fermi velocity in the physics literature corresponds to a Bloch state velocity at the conical points. From (3.27) and also Figure 6 we see that such a limit (if taken in norm) would depend on the angle from which we approach the conical points. Thus, this quantity is not well-defined in this model. Likewise, there has been some controversy about the nature of this quantity in graphene [S17]. See (7.5) for an approximation in our setting.

3.4. Different representations of the effective Hamiltonian. Since any vertex is an integer translate of either of the two vertices $r_{0}, r_{1} \in W_{\Lambda}$ by basis vectors $b_{1}, b_{2}$, we indentify $\ell^{2}(\mathcal{V}) \simeq \ell^{2}\left(\mathbb{Z}^{2} ; \mathbb{C}^{2}\right)$. Our next Lemma provides the equivalent form of $K_{\Lambda}$ (3.14) under this identification.

Lemma 3.3. The operator $K_{\Lambda}$ given by (3.14) is unitarily equivalent to an operator $Q_{\Lambda} \in \mathcal{L}\left(\ell^{2}\left(\mathbb{Z}^{2} ; \mathbb{C}^{2}\right)\right)$

$$
Q_{\Lambda}:=\frac{1}{3}\left(\begin{array}{cc}
0 & 1+\tau^{0}+\tau^{1} \\
\left(1+\tau^{0}+\tau^{1}\right)^{*} & 0
\end{array}\right)
$$

where $\tau^{0}, \tau^{1} \in \mathcal{L}\left(\ell^{2}\left(\mathbb{Z}^{2} ; \mathbb{C}\right)\right)$ are defined by

$$
\tau^{0}(r)(\gamma):=r\left(\gamma_{1}-1, \gamma_{2}\right) \quad \tau^{1}(r)(\gamma):=e^{i h \gamma_{1}} r\left(\gamma_{1}, \gamma_{2}-1\right), \quad \gamma \in \mathbb{Z}^{2}, \quad r \in \ell^{2}\left(\mathbb{Z}^{2} ; \mathbb{C}\right)
$$


and satisfy the Weyl commutation relation

$$
\tau^{1} \tau^{0}=e^{i h} \tau^{0} \tau^{1}
$$

Proof. The unitary operator eliminating the vector potential along two of the three non-equivalent edges is the multiplication operator

$$
U z:=\left(\zeta_{v} z(v)\right)_{v \in \mathcal{V}(\Lambda)}
$$

with recursively defined factors

$$
\begin{gathered}
\zeta_{r_{0}}:=1, \quad \zeta_{\gamma_{1} b_{1}+\gamma_{2} b_{2}+r_{1}}:=e^{i A_{\gamma_{1} b_{1}+\gamma_{2} b_{2}+f} \zeta_{\gamma_{1} b_{1}+\gamma_{2} b_{2}+r_{0}}} \\
\zeta_{\gamma_{1} b_{1}+\left(\gamma_{2}+1\right) b_{2}+r_{0}}:=e^{i\left(-A_{\gamma_{1} b_{1}+\left(\gamma_{2}+1\right) b_{2}+h}-h \gamma_{1}+A_{\gamma_{1} b_{1}+\gamma_{2} b_{2}+f}\right)} \zeta_{\gamma_{1} b_{1}+\gamma_{2} b_{2}+r_{0}} \\
\zeta_{\left(\gamma_{1}+1\right) b_{1}+\gamma_{2} b_{2}+r_{0}}:=e^{i\left(-A_{\left(\gamma_{1}+1\right) b_{1}+\gamma_{2} b_{2}+g}+A_{\left.\gamma_{1} b_{1}+\gamma_{2} b_{2}+f\right)} \zeta_{\gamma_{1} b_{1}+\gamma_{2} b_{2}+r_{0}} .\right.}
\end{gathered}
$$

Defining $K_{\Lambda}^{\#}:=U^{*} K_{\Lambda} U$ we see that

$$
K_{\Lambda}^{\#}(z)(v)=\frac{1}{3}\left\{\begin{array}{l}
z(v+g)+z(v+f)+e^{i h \gamma_{1}} z(v+h), v \in i(\mathcal{V}(\Lambda)) \\
z(v-g)+z(v-f)+e^{-i h \gamma_{1}} z(v-h), v \in t(\mathcal{V}(\Lambda))
\end{array}\right.
$$

where $\gamma_{1}$ is such that $v=\gamma_{1} b_{1}+\gamma_{2} b_{2}+r_{0,1}$. In order to transform $K_{\Lambda}^{\#}$ to $Q_{\Lambda}$ we use the unitary map $W: \ell^{2}(\mathcal{V}(\Lambda)) \rightarrow \ell^{2}\left(\mathbb{Z}^{2}, \mathbb{C}^{2}\right)$ defined as

$$
W z(\gamma):=\left(z\left(r_{0}+\gamma_{1} b_{1}+\gamma_{2} b_{2}\right), z\left(\gamma_{1} b_{1}+\gamma_{2} b_{2}+r_{1}\right)\right)^{T} .
$$

We conclude that, $Q_{\Lambda}=\left(U W^{*}\right)^{*} K_{\Lambda}\left(U W^{*}\right)$, proving the lemma.

Consider the matrix-valued sequence $a \in \ell^{2}\left(\mathbb{Z}^{2}, \mathbb{C}^{2}\right)$ such that

$$
\begin{gathered}
a_{(0,0)}:=\frac{1}{3}\left(\begin{array}{ll}
0 & 1 \\
1 & 0
\end{array}\right), a_{(0,1)}:=\frac{1}{3}\left(\begin{array}{ll}
0 & 1 \\
0 & 0
\end{array}\right), a_{(1,0)}:=\frac{1}{3}\left(\begin{array}{ll}
0 & 1 \\
0 & 0
\end{array}\right), \\
a_{(0,-1)}:=\frac{1}{3}\left(\begin{array}{ll}
0 & 0 \\
1 & 0
\end{array}\right) \quad a_{(-1,0)}:=\frac{1}{3}\left(\begin{array}{ll}
0 & 0 \\
1 & 0
\end{array}\right)
\end{gathered}
$$

and $a_{\beta}:=0$ for any other $\beta \in \mathbb{Z}^{2}$. Then, we can write (3.29) in the compact form

$$
Q_{\Lambda}=\sum_{\beta \in \mathbb{Z}^{2} ;|\beta| \leq 1} a_{\beta}\left(\tau^{0}\right)^{\beta_{1}}\left(\tau^{1}\right)^{\beta_{2}}
$$

We will exhibit two representations of $Q_{\Lambda}$ : the first as a magnetic matrix and then as a pseudodifferential operator. For that we follow the presentation of Helffer-Sjöstrand [HS90b]. We proceed by defining the set of rapidly decaying $\mathbb{C}^{2 \times 2}$-valued functions on $\mathbb{Z}^{2}$ :

$$
\mathscr{S}\left(\mathbb{Z}^{2}\right):=\left\{f: \mathbb{Z}^{2} \rightarrow \mathbb{C}^{2 \times 2}: \forall N \exists C_{N} \quad\|f(\gamma)\| \leq C_{N}(1+|\gamma|)^{-N}\right\}
$$


Definition 3.4 (Magnetic matrices). A function $f \in \mathscr{S}\left(\mathbb{Z}^{2}\right)$ defines a magnetic matrix

$$
A^{h}(f) \in \mathcal{L}\left(\ell^{2}\left(\mathbb{Z}^{2}, \mathbb{C}^{2}\right)\right), \quad A^{h}(f):=\left(e^{-i \frac{h}{2} \omega(\gamma, \delta)} f(\gamma-\delta)\right)_{\gamma, \delta \in \mathbb{Z}^{2}}
$$

which acts on $\ell^{2}\left(\mathbb{Z}^{2} ; \mathbb{C}^{2}\right)$ by matrix-like multiplication

$$
\left(A^{h}(f) u\right)_{\gamma}=\sum_{\delta \in \mathbb{Z}^{2}}\left(A^{h}(f)\right)_{\gamma, \delta} u_{\delta} .
$$

We now consider discrete magnetic translations $\tau_{\gamma}^{B}$ induced by the continuous magnetic translations (3.18) on the $\mathbb{Z}^{2}$-lattice $\tau_{\gamma}^{B} \in \mathcal{L}\left(\ell^{2}\left(\mathbb{Z}^{2}\right)\right)$ that are given by

$$
\tau_{\delta}^{B}(f)(\gamma):=e^{-i \frac{h}{2} \omega(\gamma, \delta)} f(\gamma-\delta), \quad \omega(\gamma, \delta):=\delta_{1} \gamma_{2}-\delta_{2} \gamma_{1}
$$

Just as $H^{B}$ commutes with the continuous magnetic translations (3.18), the magnetic matrices commute with discrete translations

$$
\left(A^{h}(f) u\right)_{\gamma}=\sum_{\delta \in \mathbb{Z}^{2}}\left(A^{h}(f)\right)_{\gamma, \delta} u_{\delta}=\sum_{\delta \in \mathbb{Z}^{2}}\left(\tau_{\delta}^{B} f\right)_{\gamma} u_{\delta}
$$

which satisfy

$$
\tau_{\gamma}^{B} \tau_{\delta}^{B}=e^{i h \omega(\gamma, \delta)} \tau_{\delta}^{B} \tau_{\gamma}^{B}
$$

Lemma 3.5. $Q_{\Lambda}$ and $A^{h}(a)$, with a given by (3.34), are unitary equivalent.

Proof. Let $u \in \ell^{2}\left(\mathbb{Z}^{2} ; \mathbb{C}^{2}\right)$, then we have

$$
\begin{aligned}
\left(Q_{\Lambda} u\right)(\gamma) & =\sum_{\delta \in \mathbb{Z}^{2} ;|\delta| \leq 1} a_{\delta} e^{i h \gamma_{1} \delta_{2}} u(\gamma-\delta)=\sum_{\delta \in \mathbb{Z}^{2} ;|\delta| \leq 1} a_{\delta} e^{-i \frac{h}{2} \delta_{1} \delta_{2}} e^{i h \gamma_{1} \delta_{2}} u(\gamma-\delta) \\
& =\sum_{\delta \in \mathbb{Z}^{2} ;|\gamma-\delta| \leq 1} a_{\gamma-\delta} e^{-i \frac{h}{2}\left(\gamma_{1}-\delta_{1}\right)\left(\gamma_{2}-\delta_{2}\right)} e^{i h \gamma_{1}\left(\gamma_{2}-\delta_{2}\right)} u(\delta) \\
& =\sum_{\delta \in \mathbb{Z}^{2} ;|\gamma-\delta| \leq 1} e^{i \frac{h}{2}\left(\gamma_{1} \gamma_{2}-\delta_{1} \delta_{2}\right)} e^{i \frac{h}{2}\left(\gamma_{2} \delta_{1}-\delta_{2} \gamma_{1}\right)} a_{\gamma-\delta} u(\delta) \\
& =\sum_{\delta \in \mathbb{Z}^{2} ;|\gamma-\delta| \leq 1} e^{i \frac{h}{2} \gamma_{1} \gamma_{2}} A_{\gamma, \delta}^{h}(a) e^{-i \frac{h}{2} \delta_{1} \delta_{2}} u(\delta) .
\end{aligned}
$$

Hence, the unitary operator $V \in \mathcal{L}\left(\ell^{2}\left(\mathbb{Z}^{2} ; \mathbb{C}^{2}\right)\right)$ acting by $V u(\gamma):=e^{-i \frac{h}{2} \gamma_{1} \gamma_{2}} u(\gamma)$, yields unitary equivalence $Q_{\Lambda}=V^{*} A^{h}(a) V$.

For $f, g \in \mathscr{S}\left(\mathbb{Z}^{2}\right)$ we define a (non-commutative) product

$$
f \#_{h} g:=A^{h}(f)(g)=A^{-h}(g)(f)=\sum_{\gamma \in \mathbb{Z}^{2}} f(\gamma)\left(\tau_{\gamma}^{-B} g\right)(\bullet) .
$$

If $f \in \mathscr{S}\left(\mathbb{Z}^{2}\right)$ then

$$
A^{h}(f)^{-1} \in \mathcal{L}\left(\ell^{2}\left(\mathbb{Z}^{2}, \mathbb{C}^{2 \times 2}\right)\right) \Longrightarrow \exists g \in \mathscr{S}\left(\mathbb{Z}^{2}\right), \quad A^{h}(f)^{-1}=A^{h}(g),
$$


and

$$
f \#_{h} g=g \#_{h} f=\mathrm{id}_{\mathbb{C}^{2 \times 2}} \delta_{0},
$$

see the proof at the end of this section and [HS89, Proposition 5.1] for a slightly different statement.

For $f \in \mathscr{S}\left(\mathbb{Z}^{2}\right)$, we define the Fourier transform as

$$
\widehat{f}(x, \xi):=\sum_{\gamma \in \mathbb{Z}^{2}} f(\gamma) e^{i\left\langle\gamma,(x, \xi)^{T}\right\rangle} \text {, such that } \widehat{f} \in C^{\infty}\left(\mathbb{T}_{*}^{2}\right) .
$$

In particular, for $a \in \mathscr{S}\left(\mathbb{Z}^{2}\right)$ given in (3.34) the Fourier transform is given by

$$
\widehat{a}(x, \xi):=\frac{1}{3}\left(\begin{array}{cc}
0 & 1+e^{i x}+e^{i \xi} \\
1+e^{-i x}+e^{-i \xi} & 0
\end{array}\right) .
$$

We observe that for $\gamma:=(1,0)$ and $\delta:=(0,1)$, equation (3.40) becomes

$$
\tau_{\gamma}^{-B} \tau_{\delta}^{-B}=e^{-i h} \tau_{\delta}^{-B} \tau_{\gamma}^{-B}
$$

In semiclassical Weyl quantization (see [Zw12, Theorem 4.7]) the same commutation relation is satisfied by

$$
\mathrm{Op}_{h}^{\mathrm{w}}\left(e^{i x}\right) \mathrm{Op}_{h}^{\mathrm{w}}\left(e^{i \xi}\right)=e^{-i h} \mathrm{Op}_{h}^{\mathrm{w}}\left(e^{i \xi}\right) \mathrm{Op}_{h}^{\mathrm{w}}\left(e^{i x}\right) .
$$

Looking at the product formula we see that when we replace $\tau_{\gamma}^{-B}$ in (3.41) by

$$
\mathrm{Op}_{h}^{\mathrm{w}}\left((x, \xi) \mapsto e^{i\left\langle\gamma,(x, \xi)^{T}\right\rangle}\right)
$$

we obtain a homomorphism

$$
\begin{gathered}
\Theta: \mathscr{S}\left(\mathbb{Z}^{2}\right) \rightarrow \mathcal{L}\left(L^{2}(\mathbb{R})\right), \quad \Theta(f):=\sum_{\gamma \in \mathbb{Z}^{2}} f(\gamma) \mathrm{Op}_{h}^{\mathrm{w}}\left((x, \xi) \mapsto e^{i\left\langle\gamma,(x, \xi)^{T}\right\rangle}\right)=\mathrm{Op}_{h}^{\mathrm{w}}(\widehat{f}), \\
\Theta\left(f \#_{h} g\right)=\Theta(f) \circ \Theta(g), \quad \Theta\left(f(-\bullet)^{*}\right)=\Theta(f)^{*} .
\end{gathered}
$$

Proof of (3.42). Invertibility of $A^{h}(f)$ on $\ell^{2}$ is equivalent to invertibility of $\mathrm{Op}_{h}^{\mathrm{w}}(\widehat{f})$. A semiclassical version of Beals's lemma, due to Helffer-Sjöstrand (see [DS99, Chapter 8] or [Zw12, Theorem 8.3]), shows that $\mathrm{Op}_{h}^{\mathrm{w}}(\widehat{f})^{-1}=\mathrm{Op}_{h}^{\mathrm{w}}(G), G \in S(1)$. We also see that $G$ has to be periodic and that implies that $G=\widehat{g}$ for $g \in \mathscr{S}$.

\section{REGULARIZED TRACES}

As recalled in $\S 1$ the density of states is defined using regularized traces of functions of the Hamiltonian. We start with a general definition:

Definition 4.1. Put $B(R):=\left\{x \in \mathbb{R}^{2}:|x|<R\right\}$ and suppose that $T \in \mathcal{L}\left(L^{2}(\mathcal{E})\right)$ has the property for all $R>0$ the operator $\mathbb{1}_{B(R)} T \mathbb{1}_{B(R)}$ is of trace-class. Then we define

$$
\tilde{\operatorname{tr}} T:=\lim _{R \rightarrow \infty} \frac{\operatorname{tr} \mathbb{1}_{B(R)} T \mathbb{1}_{B(R)}}{|B(R)|}
$$


provided this limit exists.

Similarly, for a lattice $\Gamma \subset \mathbb{R}^{2}$ and $A \in \mathcal{L}\left(\ell^{2}\left(\Gamma, \mathbb{C}^{2}\right)\right)$ given by

$$
A(s)(\gamma):=\sum_{\beta \in \mathbb{Z}^{2}} k(\gamma, \beta) s(\beta)
$$

with $k(\gamma, \beta) \in \mathbb{C}^{2 \times 2}$, we define

$$
\widehat{\operatorname{tr}}_{\Gamma} A:=\lim _{R \rightarrow \infty} \frac{1}{|B(R)|} \sum_{\gamma \in \Gamma \cap B(R)} \operatorname{tr}_{\mathbb{C}^{2}} k(\gamma, \gamma)
$$

provided the limit exists.

Remark 5. Most of the results of this section hold for both $H^{B}$ and $H^{D}$ and the proofs do not differ for the two operators. In such case we consider $H^{B}$ only.

We start with some general comments about $\widehat{\operatorname{tr}}$ :

Lemma 4.2. Let $g \in \mathscr{S}\left(\mathbb{Z}^{2}\right)$ and let $A^{h}(g)$ be the corresponding magnetic matrix (Definition 3.4). Then the regularized trace $\widehat{\operatorname{tr}}_{\mathbb{Z}^{2}}\left(A^{h}(g)\right)$ exists and is given by

$$
\widehat{\operatorname{tr}}_{\mathbb{Z}^{2}}\left(A^{h}(g)\right)=\operatorname{tr}_{\mathbb{C}^{2}}(g(0))=\frac{1}{(2 \pi)^{2}} \int_{\mathbb{T}_{*}^{2}} \operatorname{tr}_{\mathbb{C}^{2}} \widehat{g}(x, \xi) d x d \xi
$$

Proof. Since the kernels of the magnetic matrix satisfy on the diagonal $A^{h}(g)_{\gamma, \gamma}=g(0)$ the proof of this equality is immediate.

In view of this lemma we will abuse the notation slightly and introduce

Definition 4.3. Let $f \in C^{\infty}\left(\mathbb{R}^{2}\right)$ be $(2 \pi \mathbb{Z})^{2}$ periodic. Then we define the regularized trace

$$
\widehat{\operatorname{tr}}\left(\mathrm{Op}_{h}^{w}(f)\right):=\frac{1}{(2 \pi)^{2}} \int_{\mathbb{T}_{*}^{2}} \operatorname{tr}_{\mathbb{C}^{2}} f(x, \xi) d x d \xi
$$

We now show that for $f \in C_{\mathrm{c}}(\mathbb{R})$ the operators $f\left(H^{B}\right)$ and $f\left(H^{B, D}\right)$ have regularized traces. Because we are essentially in dimension one, we have stronger trace class properties:

Lemma 4.4. For $z \in \mathbb{C} \backslash \mathbb{R}$ the regularized traces of $\left(H^{\bullet}-z\right)^{-1}$ exist and

$$
\widetilde{\operatorname{tr}}\left(H^{\bullet}-z\right)^{-1}=\frac{2}{3 \sqrt{3}} \operatorname{tr} \mathbb{1}_{\mathcal{E}\left(W_{\Lambda}\right)}\left(H^{\bullet}-z\right)^{-1}, \quad \bullet=B, D .
$$

Proof. We consider $H^{B}$ only. Since $D\left(H^{B}\right) \subset H^{2}(\mathcal{E})$, we see that for $\psi \in C_{\mathrm{c}}^{\infty}\left(B_{\mathbb{R}^{2}}(0,2 R)\right)$, $\psi\left(H^{B}-z\right)^{-1}: L^{2}(\mathcal{E}) \rightarrow H^{2}\left(\mathcal{E} \cap B_{\mathbb{R}^{2}}(0,2 R)\right)$ is of trace class. (We are in dimension one here and the trace class property in dimension $n$ is obtained for maps $L^{2}\left(\mathbb{R}^{n}\right) \rightarrow$ 
$H^{s}(B(0, r)), s>n$; hence $H^{2}$ is sufficient - see for instance [DyZw2, Proposition B.20].) In addition, we have the trace norm estimate:

$$
\begin{aligned}
\left\|\psi\left(H^{B}-z\right)^{-1}\right\|_{\mathcal{L}_{1}} & \leq C_{\psi}\left\|\left(H^{B}-z\right)^{-1}\right\|_{L^{2} \rightarrow D\left(H^{B}\right)} \leq C_{\psi} \sup _{x \in \mathbb{R}}|x-z|^{-1}(1+|x|) \\
& \leq C_{\psi}(1+|\operatorname{Re} z|)|\operatorname{Im} z|^{-1}
\end{aligned}
$$

If we choose $\psi \equiv 1$ on a neighbourhood of $B_{\mathbb{R}^{2}}(0, R)$ then

$$
\mathbb{1}_{B_{\mathbb{R}^{2}}(0, R)}\left(H^{B}-z\right)^{-1}=\mathbb{1}_{B_{\mathbb{R}^{2}}(0, R)} \psi\left(H^{B}-z\right)^{-1} \in \mathcal{L}_{1}\left(L^{2}(\mathcal{E})\right) .
$$

We now choose $m_{R}, M_{R} \subset \mathbb{Z}^{2}$ such that

$$
\begin{gathered}
\Omega_{m_{R}} \subset B_{\mathbb{R}^{2}}(0, R) \cap \mathcal{E} \subset \Omega_{M_{R}}, \\
\Omega_{Q}:=\bigcup_{\gamma \in Q}\left(\mathcal{E}\left(W_{\Lambda}\right)+\gamma_{1} b_{1}+\gamma_{2} b_{2}\right), \quad\left|M_{R} \backslash m_{R}\right| \leq C R .
\end{gathered}
$$

In particular, since the area of a hexagonal cell is given by $\frac{3 \sqrt{3}}{2}$, we have

$$
\left|m_{R}\right|=\frac{2}{3 \sqrt{3}}\left|B_{\mathbb{R}^{2}}(0, R)\right|+\mathcal{O}(R) .
$$

We now write

$$
\operatorname{tr} \mathbb{1}_{B_{\mathbb{R}^{2}}(0, R)}\left(H^{B}-z\right)^{-1}=\operatorname{tr} \mathbb{1}_{\Omega_{m_{R}}}\left(H^{B}-z\right)^{-1}+\operatorname{tr} \mathbb{1}_{B_{\mathbb{R}^{2}}(0, R) \backslash \Omega_{m_{R}}}\left(H^{B}-z\right)^{-1} .
$$

Using (3.22) we get

$$
\begin{aligned}
T_{\gamma}^{B} \mathbb{1}_{\mathcal{E}\left(W_{\Lambda}\right)} T_{-\gamma}^{B} f & =T_{\gamma}^{B} \mathbb{1}_{\mathcal{E}\left(W_{\Lambda}\right)} u^{B}(-\gamma) f\left(\bullet+\gamma_{1} b_{1}+\gamma_{2} b_{2}\right) \\
& =\mathbb{1}_{\mathcal{E}\left(W_{\Lambda}\right)+\gamma_{1} b_{1}+\gamma_{2} b_{2}} f
\end{aligned}
$$

so that we can expand the first term on the right hand side of (4.9) as follows

$$
\begin{aligned}
\operatorname{tr} \mathbb{1} \Omega_{m_{R}}\left(H^{B}-z\right)^{-1} & =\sum_{\gamma \in m_{R}} \operatorname{tr} \mathbb{1}_{\mathcal{E}\left(W_{\Lambda}\right)+\gamma_{1} b_{1}+\gamma_{2} b_{2}}\left(H^{B}-z\right)^{-1} \\
& =\sum_{\gamma \in m_{R}} \operatorname{tr} T_{\gamma}^{B} \mathbb{1}_{\mathcal{E}\left(W_{\Lambda}\right)} T_{-\gamma}^{B}\left(H^{B}-z\right)^{-1} \\
& =\left|m_{R}\right| \operatorname{tr} \mathbb{1}_{\mathcal{E}\left(W_{\Lambda}\right)}\left(H^{B}-z\right)^{-1}
\end{aligned}
$$

Here we used (3.24) and the cyclicity of the trace. 
To estimate the second term in (4.9) we write

$$
\begin{aligned}
\left\|\mathbb{1}_{B_{\mathbb{R}^{2}}(0, R) \backslash \Omega_{m_{R}}}\left(H^{B}-z\right)^{-1}\right\|_{\mathcal{L}_{1}} & \leq\left\|\mathbb{1}_{\Omega_{M_{R}} \backslash \Omega_{m_{R}}}\left(H^{B}-z\right)^{-1}\right\|_{\mathcal{L}_{1}} \\
& \leq \sum_{\gamma \in M_{R} \backslash m_{R}}\left\|\mathbb{1}_{\mathcal{E}\left(W_{\Lambda}\right)+\gamma_{1} b_{1}+\gamma_{2} b_{2}}\left(H^{B}-z\right)^{-1}\right\|_{\mathcal{L}_{1}} \\
& \leq \sum_{\gamma \in M_{R} \backslash m_{R}}\left\|T_{\gamma}^{B} \mathbb{1}_{\mathcal{E}\left(W_{\Lambda}\right)} T_{-\gamma}^{B}\left(H^{B}-z\right)^{-1}\right\|_{\mathcal{L}_{1}} \\
& =\left|M_{R} \backslash m_{R}\right|\left\|\mathbb{1}_{\mathcal{E}\left(W_{\Lambda}\right)}\left(H^{B}-z\right)^{-1}\right\|_{\mathcal{L}_{1}} \\
& \leq C R(1+|\operatorname{Re} z|)|\operatorname{Im} z|^{-1},
\end{aligned}
$$

where we used (4.6) and (4.7). Returning to (4.9) we see that (4.5) follows from (4.8) and (4.11).

We now consider regularized traces of $f\left(H^{B}\right)$ and $f\left(H^{D}\right)$ and we will use the functional calculus of Helffer-Sjöstrand. For that we recall that for any $f \in C_{\mathrm{c}}^{\infty}(\mathbb{R})$ can be extended to $\tilde{f} \in \mathscr{S}(\mathbb{C})$ such that $\left.\widetilde{f}\right|_{\mathbb{R}}=f$ and $\partial_{\bar{z}} \widetilde{f}=\mathcal{O}\left(|\operatorname{Im} z|^{\infty}\right)$. The function $\tilde{f}$ is a then called an almost analytic extension of $f$. A compact formula for $\tilde{f}$ was given by Mather and Jensen-Nakamura:

$$
\begin{aligned}
& \widetilde{f}(x+i y)=\frac{1}{2 \pi} \chi(y) \psi(x) \int_{\mathbb{R}} \chi(y \xi) \widehat{f}(\xi) e^{i(x+i y) \xi} d \xi, \\
& \chi, \psi \in C_{\mathrm{c}}^{\infty}(\mathbb{R}),\left.\quad \psi\right|_{\operatorname{supp} f+(-1,1)}=1,\left.\quad \chi\right|_{(-1,1)}=1,
\end{aligned}
$$

see for instance [DS99, Chapter 8]. The relevance of this construction here comes from the Helffer-Sjöstrand formula: for any self-adjoint operator $P$,

$$
f(P)=\frac{1}{\pi} \int_{\mathbb{C}} \partial_{\bar{z}} \tilde{f}(z)(P-z)^{-1} d m(z)
$$

where $\lambda_{\mathbb{C}}$ is the Lebesgue measure on $\mathbb{C}$. The integral on the right hand side is welldefined as $\partial_{\bar{z}} \widetilde{f}(z)=\mathcal{O}\left(|\operatorname{Im} z|^{\infty}\right)$ and $\left\|(P-z)^{-1}\right\|=\mathcal{O}(1 /|\operatorname{Im} z|)$, by self-adjointness.

The proof of Lemma 4.4 and the dominated convergence theorem based on (4.6),(4.11) and (4.12), immediately give

Lemma 4.5. Let $f \in C_{c}(\mathbb{R})$ then $\widetilde{\operatorname{tr}}\left(f\left(H^{\bullet}\right)\right)$ exist and

$$
\tilde{\operatorname{tr}} f\left(H^{\bullet}\right)=\frac{2}{3 \sqrt{3}} \operatorname{tr} \mathbb{1}_{\mathcal{E}\left(W_{\Lambda}\right)} f\left(H^{\bullet}\right) .
$$

The lemma allows a rigorous definition of the density of states measure: the functional $C_{c}(\mathbb{R}) \ni f \mapsto \widetilde{\operatorname{tr}}\left(f\left(H^{B}\right)\right)$ is positive. Thus, by the Riesz-Markov theorem, it defines a Radon measure:

Definition 4.6 (Density of states measure). The density of states $\rho_{B} \in \mathscr{D}^{\prime 0}(\mathbb{R})$ is the Radon measure such that

$$
\widetilde{\operatorname{tr}}\left(f\left(H^{B}\right)\right)=\int_{\mathbb{R}} f(x) \rho_{B}(x) d x
$$


where we use the informal notation for the action of distributions of order zero on function (see $[\mathrm{Ho} 03, \S 2.1]$ ) The distribution function of the measure $\rho_{B}$ is called the integrated density of states.

In Krein's resolvent formula (3.15) the auxiliary operators $\Lambda^{B}$ and $\Lambda^{D}$ appear instead of $H^{B}$ and $H^{D}$. The following Lemma shows that their regularized traces coincide.

Lemma 4.7. For $f \in C_{c}(\mathbb{R})$,

$$
\widetilde{\operatorname{tr}}\left(f\left(\Lambda^{\bullet}\right)\right)=\widetilde{\operatorname{tr}}\left(f\left(H^{\bullet}\right)\right), \quad \bullet=B, D .
$$

Proof. By the functional calculus, the unitary Peierls' substitution $P$ satisfies (3.6)

$$
f\left(\Lambda^{B}\right)=P^{-1} f\left(H^{B}\right) P .
$$

Since $P$ and $P^{-1}$ are just multiplication operators

$$
\begin{aligned}
\operatorname{tr}\left(\mathbb{1}_{B(R)} f\left(\Lambda^{B}\right) \mathbb{1}_{B(R)}\right) & =\operatorname{tr}\left(\begin{array}{lllll}
P & \mathbb{1}_{B(R)} & f\left(\Lambda^{B}\right) & \mathbb{1}_{B(R)} & P^{-1}
\end{array}\right) \\
& =\operatorname{tr}\left(\begin{array}{lllll}
P & \mathbb{1}_{B(R)} & P^{-1} f\left(H^{B}\right) P & \mathbb{1}_{B(R)} & P^{-1}
\end{array}\right) \\
& =\operatorname{tr}\left(\begin{array}{llll}
\mathbb{1}_{B(R)} & f\left(H^{B}\right) & \left.\mathbb{1}_{B(R)}\right) .
\end{array}\right.
\end{aligned}
$$

Lemma 4.5 shows the existence of the regularized trace then.

We now combine (4.14) with Krein's formula (3.15) to see that

$$
\begin{aligned}
f\left(\Lambda^{B}\right) & =\frac{1}{\pi} \int_{\mathbb{C}} \partial_{\bar{z}} \tilde{f}(z)\left(\left(\Lambda^{D}-z\right)^{-1}-\gamma(z) M(z)^{-1} \gamma(\bar{z})^{*}\right) d m(z) \\
& =f\left(\Lambda^{D}\right)-\frac{1}{\pi} \int_{\mathbb{C}} \partial_{\bar{z}} \tilde{f}(z) \gamma(z) M(z)^{-1} \gamma(\bar{z})^{*} d m(z) .
\end{aligned}
$$

Using Lemma 4.7, we can apply the operator $\widetilde{\text { tr }}$ to the preceding equation and obtain

$$
\widetilde{\operatorname{tr}} f\left(H^{B}\right)=\widetilde{\operatorname{tr}} f\left(\Lambda^{D}\right)-\frac{1}{\pi} \widetilde{\operatorname{tr}} \int_{\mathbb{C}} \partial_{\bar{z}} \widetilde{f}(z) \gamma(z) M(z)^{-1} \gamma(\bar{z})^{*} d m(z) .
$$

In the following, we will systematically analyze the terms on the right side. We start with the term containing operator $\Lambda^{D}$.

Lemma 4.8. The contribution $\widetilde{\operatorname{tr}}\left(f\left(\Lambda^{D}\right)\right)$ of the Dirichlet operator $\Lambda^{D}$ is given by

$$
\tilde{\operatorname{tr}} f\left(\Lambda^{D}\right)=\frac{2}{\sqrt{3}} \sum_{\lambda \in \operatorname{Spec}\left(\Lambda_{(0,1)}^{D}\right)} f(\lambda)
$$

where $\Lambda_{(0,1)}^{D}: H_{0}^{1}(0,1) \cap H^{2}(0,1) \subset L^{2}(0,1) \rightarrow L^{2}(0,1)$ with $\Lambda_{(0,1)}^{D} \psi:=-\psi^{\prime \prime}+V \psi$.

Proof. Let $\Lambda^{D}=\sum_{\lambda \in \operatorname{Spec}\left(\Lambda_{(0,1)}^{D}\right)}^{\infty} \lambda P_{\operatorname{ker}\left(\Lambda^{D}-\lambda\right)}$ be the spectral decomposition of $\Lambda^{D}$ where $P_{\operatorname{ker}\left(\Lambda^{D}-\lambda\right)}$ is the orthogonal projection onto the infinite dimensional space $\operatorname{ker}\left(\Lambda^{D}-\lambda\right)$. The spectral theorem implies $f\left(\Lambda^{D}\right)=\sum_{\lambda \in \operatorname{Spec}\left(\Lambda_{(0,1)}^{D}\right)}^{\infty} f(\lambda) P_{\operatorname{ker}\left(\Lambda^{D}-\lambda\right)}$, which is a finite 
sum, as the eigenvalues of the Dirichlet operator tend to infinity. Thus, since each edge carries precisely one non-degenerate eigenfunction for every eigenvalue $\lambda \in \operatorname{Spec}\left(\Lambda^{D}\right)$,

$$
\begin{aligned}
\tilde{\operatorname{tr}} f\left(\Lambda^{D}\right) & =\lim _{R \rightarrow \infty} \frac{\operatorname{tr}\left(\mathbb{1}_{B(R)} f\left(\Lambda^{D}\right) \mathbb{1}_{B(R)}\right)}{|B(R)|} \\
& =\sum_{\lambda \in \operatorname{Spec}\left(\Lambda_{(0,1)}^{D}\right)} f(\lambda) \lim _{R \rightarrow \infty} \frac{\operatorname{tr}\left(\mathbb{1}_{B(R)} P_{\operatorname{ker}\left(\Lambda^{D}-\lambda\right)} \mathbb{1}_{B(R)}\right)}{|B(R)|}=\frac{2}{\sqrt{3}} \sum_{\lambda \in \operatorname{Spec}\left(\Lambda_{(0,1)}^{D}\right)} f(\lambda),
\end{aligned}
$$

with $\frac{2}{\sqrt{3}}$ being the ratio of edges per unit volume.

We now move to the second term in (4.20). In particulare we eliminate the gamma field in our expressions.

Lemma 4.9. With $M(z)$ defined in (3.13) we have

$$
\widetilde{\operatorname{tr}} \int_{\mathbb{C}} \partial_{\bar{z}} \widetilde{f}(z) \gamma(z) M(z)^{-1} \gamma(\bar{z})^{*} d m(z)=\int_{\mathbb{C}} \partial_{\bar{z}} \widetilde{f}(z) \widehat{\operatorname{tr}}_{\mathcal{V}(\Lambda)} \partial_{z} M(z) M(z)^{-1} d m(z) .
$$

Proof. The estimates in the proof of Lemma 4.4 show that we can move $\widetilde{\text { r }}$ inside of the integral on the left hand side. Together with (3.16) this means that it suffices to prove that

$$
\widetilde{\operatorname{tr}}\left(\gamma(z) M(z)^{-1} \gamma(\bar{z})^{*}\right)=\widehat{\operatorname{tr}}_{\mathcal{V}(\Lambda)}\left(\gamma(\bar{z})^{*} \gamma(z) M(z)^{-1}\right), \quad z \in \mathbb{C} \backslash \mathbb{R} .
$$

This identity can now be shown by verifying the conditions of the third statement in [HS89, Proposition 7.1] with

$$
C:=\gamma(z) M(z)^{-1}, \quad D:=\gamma(\bar{z})^{*}
$$

but we present a different argument.

Using the unitary Peierls operator $P$ (3.5) magnetic translations (3.18), and operators $\pi$ and $\gamma(z)$ from (3.10),(3.11) we define modified magnetic translations (note that $z \notin \mathbb{R})$ as the following unitary operators:

$$
S_{\delta}^{B}:=P^{-1} T_{\delta}^{B} P \in \mathcal{U}\left(L^{2}(\mathcal{E})\right), \quad \sigma_{\delta}^{B}:=\pi S_{\delta}^{B} \gamma(z) \in \mathcal{U}\left(\ell^{2}(\mathcal{V})\right),
$$

where we note that $\sigma_{\delta}^{B}$ does not depend on $z$.

To see that $\sigma_{\delta}^{B}$ is unitary we first note that $\left(\sigma_{\delta}^{B}\right)^{-1}=\sigma_{-\delta}^{B}$ and that it is an isometry (see (3.18) and (3.22) for definitions of $T_{\gamma}^{B}$ and $\left.u^{B}(\gamma)\right)$ :

$$
\begin{aligned}
\left\|\sigma_{\delta}^{B} w\right\|^{2} & =\sum_{v \in \mathcal{V}(\Lambda)}\left|\left(\pi S_{\delta}^{B} \gamma(z) w\right)(v)\right|^{2}=\sum_{v \in \mathcal{V}(\Lambda)}\left|\left(P^{-1} T_{\delta}^{B} P \gamma(z) w\right)(v)\right|^{2} \\
& =\sum_{v \in \mathcal{V}(\Lambda)}\left|\left(u^{B}(\delta) P \gamma(z) w\right)\left(v-\delta_{1} b_{1}-\delta_{2} b_{2}\right)\right|^{2}=\sum_{v \in \mathcal{V}(\Lambda)}|(\gamma(z) w)(v)|^{2} \\
& =\sum_{v \in \mathcal{V}(\Lambda)}|w(v)|^{2}=\|w\|^{2}
\end{aligned}
$$


We now claim that $M(z)^{-1}$ commutes with $\sigma_{\gamma}^{B}$. In fact, since $H^{B}$ (3.4) and $H^{D}$ (3.7) commute with magnetic translations $T_{\delta}^{B}$, we see that $(3.5), \Lambda^{B}$ and $\Lambda^{D}$ commute then with $S_{\delta}^{B}$. The Krein formula (3.15) then implies that $S_{\delta}^{B}\left(\gamma(z) M(z)^{-1} \gamma(\bar{z})^{*}\right)=$ $\left(\gamma(z) M(z)^{-1} \gamma(\bar{z})^{*}\right) S_{\delta}^{B}$. Multiplying with the inverse of $\gamma(z)$ and $\gamma(\bar{z})^{*}$ from both sides respectively, it follows that

$$
\sigma_{\delta}^{B} M(z)^{-1}=\left(\pi S_{\delta}^{B} \gamma(z)\right) M(z)^{-1}=M(z)^{-1}\left(\gamma(\bar{z})^{*} S_{\delta}^{B} \pi^{*}\right)=M(z)^{-1} \sigma_{\delta}^{B} .
$$

In the notation of (4.23) we then see that

$$
S_{\delta}^{B} C=S_{\delta}^{B} \gamma(z) M(z)^{-1}=\gamma(z) \sigma_{\delta}^{B} M(z)^{-1}=\gamma(z) M(z)^{-1} \sigma_{\delta}^{B}=C \sigma_{\delta}^{B}
$$

and

$$
\sigma_{\delta}^{B} D=\left(\gamma(\bar{z}) \sigma_{-\delta}^{B}\right)^{*}=\left(\gamma(\bar{z}) \pi S_{-\delta}^{B} \gamma(\bar{z})\right)^{*}=\left(S_{-\delta}^{B} \gamma(\bar{z})\right)^{*}=\gamma(\bar{z})^{*} S_{\delta}^{B}=D S_{\delta}^{B}
$$

As in the proof of Lemma 4.4,

$$
\begin{aligned}
\tilde{\operatorname{tr} C D} & =\frac{2}{3 \sqrt{3}} \operatorname{tr}_{L^{2}(\mathcal{E})} \mathbb{1}_{\mathcal{E}\left(W_{\Lambda}\right)} C D=\frac{2}{3 \sqrt{3}} \operatorname{tr}_{\ell^{2}(\mathcal{V})} D \mathbb{1}_{\mathcal{E}\left(W_{\Lambda}\right)} C \\
& =\frac{2}{3 \sqrt{3}} \sum_{\gamma \in \mathbb{Z}^{2}} \sum_{v \in \mathcal{V}\left(W_{\Lambda}\right)}\left[\sigma_{\gamma}^{B} D \mathbb{1}_{\mathcal{E}\left(W_{\Lambda}\right)} C \sigma_{-\gamma}^{B}\right](v)
\end{aligned}
$$

where for an operator $A$ on $\ell^{2}(\mathcal{V})$ we write $A u(\gamma)=\sum_{\alpha \in \mathcal{V}}[A](\gamma, \alpha) u(\alpha)$. Using (4.25), (4.26) and (3.22) we then obtain

$$
\begin{aligned}
\tilde{\operatorname{tr}} C D & =\frac{2}{3 \sqrt{3}} \sum_{\gamma \in \mathbb{Z}^{2}} \sum_{v \in \mathcal{V}\left(W_{\Lambda}\right)}\left[D \mathbb{1}_{\mathcal{E}\left(W_{\Lambda}\right)+\gamma_{1} b_{1}+\gamma_{2} b_{2}} C\right](v, v) \\
& =\frac{2}{3 \sqrt{3}} \sum_{v \in \mathcal{V}\left(W_{\Lambda}\right)}\left[D I_{L^{2}(\mathcal{E})} C\right](v, v)=\frac{2}{3 \sqrt{3}} \sum_{v \in \mathcal{V}\left(W_{\Lambda}\right)}[D C](v, v) .
\end{aligned}
$$

Since $D C$ commutes with $\sigma_{\delta}^{B}$ it is unitarily equivalent to a magnetic matrix which in view of Lemma 4.2 and a lattice identification means that

$$
\widehat{\operatorname{tr}}_{\mathcal{V}(\Lambda)} D C=\frac{2}{3 \sqrt{3}} \sum_{v \in \mathcal{V}\left(W_{\Lambda}\right)}[D C](v, v)
$$

This proves (4.22) which as explained in the beginning concludes the proof.

We can now combine Lemmas 4.8,4.9 and the Krein formula to obtain

Lemma 4.10. Using (3.13) and (3.14) define.

$$
W(z):=s_{z}(1) M(z)=K_{\Lambda}-\Delta(z) .
$$

Then for $f \in C_{\mathrm{c}}^{\infty}(\mathbb{R})$ with an almost analytic extension $(4.13), \tilde{f} \in C_{\mathrm{c}}^{\infty}(\mathbb{C})$,

$$
\widetilde{\operatorname{tr}}\left(f\left(H^{B}\right)\right)=-\frac{1}{\pi} \int_{\mathbb{C}} \partial_{\bar{z}} \widetilde{f}(z) \widehat{\operatorname{tr}} \mathcal{V} \partial_{z} W(z) W(z)^{-1} d m(z)+\frac{2}{3 \sqrt{3}} \sum_{\lambda \in \operatorname{Spec}\left(\Lambda_{(0,1)}^{D}\right)} f(\lambda) .
$$


Proof. Since $\widehat{\operatorname{tr}} I_{\ell^{2}(\mathcal{V})}=\frac{4}{3 \sqrt{3}}$ (the number of vertices per unit volume) we have

$$
\widehat{\operatorname{tr}} \partial_{z} M(z) M(z)^{-1}=-\frac{4}{3 \sqrt{3}} \partial_{z} s_{z}(1) s_{z}(1)^{-1}+\widehat{\operatorname{tr}} \partial_{z} W(z) W(z)^{-1}
$$

Since the zeros of $z \mapsto s_{z}(1)$ are given by the eigenvalues of $\Lambda_{(0,1)}^{D}$, the Cauchy formula [Ho03, (3.1.11)] shows that

$$
\frac{1}{\pi} \int_{\mathbb{C}} \partial_{\bar{z}} \widetilde{f}(z) \partial_{z} s_{z}(1) s_{z}(1)^{-1} d m(z)=\sum_{\lambda \in \operatorname{Spec}\left(\Lambda_{(0,1)}^{D}\right)} f(\lambda)
$$

Combining this with (4.20), (4.21), (4.9) and (4.29) proves (4.28).

Remark 6. The Dirichlet spectrum contribution has a straightforward interpretation in the absence of magnetic fields. In that case, there is precisely one hexagonal eigenstate per fundamental cell. The ratio of fundamental cells per ball $B(R)$ scales exactly like $\frac{2}{3 \sqrt{3}}$ in the $R \rightarrow \infty$ limit which coincides with the pre-factor determined in (4.28).

We now proceed to the reduction to the effective Hamiltonian,

$$
Q^{\mathrm{w}}(x, h D)-\Delta(z), \quad Q^{\mathrm{w}}(x, h D):=\frac{1}{3}\left(\begin{array}{cc}
0 & 1+e^{i x}+e^{i h D_{x}} \\
1+e^{-i x}+e^{-i h D_{x}} & 0
\end{array}\right),
$$

which is the semiclassical quantization of

$$
Q(x, \xi):=\frac{1}{3}\left(\begin{array}{cc}
0 & 1+e^{i x}+e^{i \xi} \\
1+e^{-i x}+e^{-i \xi} & 0
\end{array}\right)
$$

The regularized trace, $\widehat{\operatorname{tr}}_{\mathcal{V}}$, in (4.28) can be expressed in terms of the regularized trace from Definition 4.3 of pseudodifferential operators $Q^{\mathrm{w}}$ :

Lemma 4.11. In the notation of Definition 4.3, Lemma 4.10 and (4.30) we have

$$
\widehat{\operatorname{tr}}_{\mathcal{V}} W^{\prime}(z) W(z)^{-1}=-\frac{2}{3 \sqrt{3}} \Delta^{\prime}(z) \widehat{\operatorname{tr}}\left(Q^{\mathrm{w}}(x, h D)-\Delta(z)\right)^{-1}, \quad z \in \mathbb{C} \backslash \mathbb{R} .
$$

Proof. The explicit unitary transformation in Lemma 3.5 shows that we can identify $W(z)$ with a magnetic matrix $A^{h}(a-\Delta(z))$ where $a$ is given by (3.34). The limiting density of vertices in the hexagonal lattice is given by $\frac{4}{3 \sqrt{3}}$ and half of this number corresponds to translates of each of $r_{0}$ and $r_{1}$. Hence,

$$
\widehat{\operatorname{tr}}_{\mathcal{V}}\left(W^{\prime}(z) W(z)^{-1}\right)=-\Delta^{\prime}(z) \frac{2}{3 \sqrt{3}} \widehat{\operatorname{tr}}_{\mathbb{Z}^{2}}\left(\left(A^{h}(a-\Delta(z))\right)^{-1}\right)
$$

We note that by (3.42) for $z \notin \mathbb{R},\left(A^{h}(a-\Delta(z))\right)^{-1}$ is also a magnetic matrix. Formula (4.32) then follows from Lemma 4.2 and Definition (4.3).

Putting all this together we obtain the main result of this section: 
Proposition 4.12. For $f \in C_{\mathrm{c}}^{\infty}(\mathbb{R})$ with an almost analytic extension (4.13), $\tilde{f} \in$ $C_{\mathrm{c}}^{\infty}(\mathbb{R})$, we have

$$
\begin{gathered}
\tilde{\operatorname{tr}}\left(f\left(H^{B}\right)\right)=\frac{2}{3 \sqrt{3} \pi} \int_{\mathbb{C}} \partial_{\bar{z}} \widetilde{f}(z) \Delta^{\prime}(z) \widehat{\operatorname{tr}}\left(Q^{\mathrm{w}}(x, h D)-\Delta(z)\right)^{-1} d m(z) \\
+\frac{2}{3 \sqrt{3}} \sum_{\lambda \in \operatorname{Spec}\left(\Lambda_{(0,1)}^{D}\right)} f(\lambda),
\end{gathered}
$$

where $Q(x, \xi)$ is given by (4.31) and $\Delta(z)$ by (2.15).

\section{Analysis of the effective Hamiltonian}

We now study the effective Hamiltonian (4.30) for $z$ near $z_{0}$ with $\Delta\left(z_{0}\right)=0$. The goal is to obtain asymptotics of of the renormalized trace of $\left(Q^{\mathrm{w}}-\Delta(z)\right)^{-1}$ - see Theorem 6.1 where for the moment we replace $\Delta(z)$ by $z$. For that we use the strategy of Helffer-Sjöstrand outlined in [HS90b, §8] but rather than follow [HS88, §2] and other numerous references cited in [HS90b, §8] we present direct arguments.

We start with some elementary analysis of the symbol $Q$ given in (4.31). Its determinant is given by $-\left|1+e^{i x}+e^{i \xi}\right|^{2} / 9$, and it vanishes at

$$
(x, \xi) \in \mathbb{Z}_{*}^{2} \pm\left(\frac{2 \pi}{3},-\frac{2 \pi}{3}\right),
$$

that is, at the Dirac points.

We consider neighbourhoods of $\pm\left(\frac{2 \pi}{3},-\frac{2 \pi}{3}\right)$ and make a symplectic change of variables:

$$
y=a(x+\xi), \quad \eta=b\left(\xi-x \pm \frac{4 \pi}{3}\right), \quad 2 a b=1
$$

we see that

$$
\begin{array}{r}
1+e^{i x}+e^{i \xi}=c(\eta \mp i y)+\mathcal{O}\left(y^{2}+\eta^{2}\right), \\
1+e^{-i x}+e^{-i \xi}=c(\eta \pm i y)+\mathcal{O}\left(y^{2}+\eta^{2}\right),
\end{array}
$$

where $c=3^{\frac{1}{4}} 2^{-\frac{1}{2}}$ and we chose $a= \pm 2^{-\frac{3}{4}} 3^{-\frac{1}{4}}$ and $b= \pm 2^{-\frac{1}{4}} 3^{\frac{1}{4}}$.

To study regularized traces of the resolvent of $Q(x, h D)$ we introduce a localized operator with discrete spectrum near 0: Its Weyl symbol is given by

$$
\begin{gathered}
Q_{0}(x, \xi):=Q(x, \xi)+\left(\begin{array}{cc}
-1+\chi_{0}(x, \xi) & 0 \\
0 & 1-\chi_{0}(x, \xi)
\end{array}\right), \\
\chi_{0} \in C_{\mathrm{c}}^{\infty}\left(\mathbb{R}^{2} ;[0,1]\right), \quad \chi_{0}(\rho)=\chi_{0}(-\rho), \quad \chi_{0}(\rho)=\left\{\begin{array}{cc}
1, & \|\rho\|_{\infty}<\pi+\frac{1}{10}, \\
0, & \|\rho\|_{\infty}>\pi+\frac{2}{10},
\end{array}\right.
\end{gathered}
$$

where $\rho=(x, \xi)$. 
We observe that for any $\delta>0$, there exists $\varepsilon>0$ such that

$$
\operatorname{det} Q_{0}(x, \xi)<-\varepsilon \text { for }\left|x \mp \frac{2 \pi}{3}\right|+\left|\xi \pm \frac{2 \pi}{3}\right|>\delta \text {. }
$$

This means that $\operatorname{det}\left(Q_{0}(x, \xi)-z\right) \in S(1)$ is elliptic (in the sense of [Zw12, §4.7.1]) away from neighbourhoods of $\pm\left(\frac{2 \pi}{3},-\frac{2 \pi}{3}\right)$ and for $z$ in a neighbourhood of 0 .

We also use microlocal weights defined as follows (see [Zw12, §8.2]):

$$
\begin{gathered}
G(x, \xi)=\frac{1}{2} \log \left(1+\xi^{2}+x^{2}\right), \quad G^{\mathrm{w}}=G^{\mathrm{w}}(x, h D), \\
e^{ \pm N G^{\mathrm{w}}}=s_{N}(x, h D, h), \quad s_{N} \in S\left(\left(1+\xi^{2}+x^{2}\right)^{ \pm N / 2}\right) .
\end{gathered}
$$

Proposition 5.1. For $\delta_{0}>0$ small enough, the spectrum of $Q_{0}^{\mathrm{w}}(x, h D)$ in $\left[-\delta_{0}, \delta_{0}\right]$ is discrete and

$$
\operatorname{Spec}\left(Q_{0}^{\mathrm{w}}(x, h D)\right) \cap\left[-\delta_{0}, \delta_{0}\right]=\left\{\kappa(n h, h)+\mathcal{O}\left(h^{\infty}\right): n \in \mathbb{Z}\right\} \cap\left[-\delta_{0}, \delta_{0}\right],
$$

with eigenvalues of multiplicity $2, \kappa(-\zeta, h)=-\kappa(\zeta, h)$, and

$$
\begin{gathered}
F\left(\kappa(\zeta, h)^{2}, h\right)=|\zeta|+\mathcal{O}\left(h^{\infty}\right), \quad F(\omega, h) \sim F_{0}(\omega)+\sum_{j=2}^{\infty} h^{j} F_{j}(\omega), \quad F_{j} \in C^{\infty}(\mathbb{R}), \\
F_{0}(\omega)=\frac{1}{4 \pi} \int_{\gamma_{\omega}} \xi d x, \quad \gamma_{\omega}=\left\{(x, \xi) \in \mathbb{T}_{*}^{2}: \frac{\left|1+e^{i x}+e^{i \xi}\right|^{2}}{9}=\omega\right\}, \quad F_{j}(0)=0,
\end{gathered}
$$

where $\gamma_{\omega}$ is oriented clockwise in the $(x, \xi)$ plane.

Moreover, the orthonormal set of eigenfunctions, $\left(u_{n}^{+}(h)\right)_{n \in \mathbb{Z}} \cup\left(u_{n}^{-}(h)\right)_{n \in \mathbb{Z}}$, satisfies

$$
Q_{0}^{\mathrm{w}}\left(x, h D, z_{0}\right) u_{n}^{ \pm}(h)=\kappa(n h, h) u_{n}^{ \pm}(h), \quad \mathrm{WF}_{h}\left(u_{n}^{ \pm}\right) \subset \operatorname{nbhd}\left( \pm\left(\frac{2 \pi}{3},-\frac{2 \pi}{3}\right)\right),
$$

and, for all $N$,

$$
\begin{gathered}
\left\|\left(1-\chi_{0}^{\mathrm{w}}(x, h D)\right) e^{N G^{\mathrm{w}}(x, h D)} u_{n}^{ \pm}(h)\right\|=\mathcal{O}_{N}\left(h^{\infty}\right), \\
\left\|e^{N G^{\mathrm{w}}(x, h D)}\left(1-\chi_{0}^{\mathrm{w}}(x, h D)\right) u_{n}^{ \pm}(h)\right\|=\mathcal{O}_{N}\left(h^{\infty}\right),
\end{gathered}
$$

where $\chi_{0}$ is defined in (5.2) and $G$ in (5.4).

Proof. We start by showing that for $\delta_{0}$ small enough the spectrum of $Q_{0}^{\mathrm{w}}$ in $\left[-\delta_{0}, \delta_{0}\right]$ is discrete and that the eigenfunctions are localized to neighbourhoods in the sense of (5.7) and (5.8). For that we define $Q_{1}:=Q+\operatorname{diag}(-1,1)$. Then $Q_{0}=Q_{1}+$ $\operatorname{diag}\left(\chi_{0},-\chi_{0}\right)$ and $Q_{1}-z$ is elliptic in $S(1)$ for $|z|$ small enough. That implies that for $0<h<h_{0},\left(Q_{1}^{\mathrm{w}}-z\right)^{-1}=\mathcal{O}(1)_{L^{2} \rightarrow L^{2}}$ in $h-$ see [Zw12, §4.7.1]. It follows that

$$
Q_{0}^{\mathrm{w}}-z=\left(Q_{1}^{\mathrm{w}}-z\right)(\mathrm{id}+K(z)), \quad K(z):=\left(Q_{1}^{\mathrm{w}}-z\right)^{-1} \operatorname{diag}\left(\chi_{0}^{\mathrm{w}},-\chi_{0}^{\mathrm{w}}\right) .
$$

Since $\chi_{0}^{\mathrm{w}}$ is a compact operator on $L^{2}$ (see [Zw12, Theorem 4.26]) we can use analytic Fredholm theory (see [Zw12, Theorem D.4]) to show that $(\mathrm{id}+K(z))^{-1}$ is meromorphic. 
That shows that $\left(Q_{0}^{\mathrm{w}}-z\right)^{-1}$ is meromorphic for $|z|$ small, that it has a discrete set of poles there, which in turn means that the spectrum near 0 is discrete.

The comment after (5.3) and [Zw12, §8.4] give the localization of eigenfunctions in (5.7). To see (5.8) we consider the conjugated operator

$$
Q_{G}^{\mathrm{w}}-z:=e^{N G^{\mathrm{w}}}\left(Q_{0}^{\mathrm{w}}-z\right) e^{-N G^{\mathrm{w}}} .
$$

From [Zw12, Theorems 4.18 and 8.6] we see that $Q_{G} \in S(1)$ and that $Q_{G}=Q_{0}+$ $\mathcal{O}_{N}(h)_{S(1)}$. Hence $Q_{G}-z$ is elliptic where $Q_{0}-z$ is elliptic and in particular near the support of $1-\chi_{0}$. Since $\left(Q_{G}^{\mathrm{w}}-z\right) e^{N G^{\mathrm{w}}} u=0, z \in \operatorname{Spec}\left(Q_{0}^{\mathrm{w}}\right), u$ an eigenfunction, the first estimate in (5.8) follows. To see the second estimate we use the wave front set estimate (5.7) and the fact that the essential support (see [Zw12, §8.4]) of the commutator of $\chi_{0}^{\mathrm{w}}$ and $e^{s G^{\mathrm{w}}}$ is supported away from $\mathrm{WF}_{h}\left(u_{n}^{ \pm}\right)$.

This means that to approximate eigenvalues of $Q_{0}^{\mathrm{w}}(x, h D)$ we need to find all microlocal solutions $(u, z)$ (that is solutions modulo $\mathcal{O}\left(h^{\infty}\right)$ ) such that $u$ satisfies $(5.8)$ and

$$
\left(Q^{\mathrm{w}}-z\right) u=\mathcal{O}\left(h^{\infty}\right), \quad \mathrm{WF}_{h}(u) \subset \operatorname{nbhd}\left( \pm\left(\frac{2 \pi}{3},-\frac{2 \pi}{3}\right)\right) .
$$

Here we replaced $Q_{0}$ by $Q$ since the corresponding operators are microlocally the same near $\pm\left(\frac{2 \pi}{3},-\frac{2 \pi}{3}\right)$ (see $[\mathrm{Zw} 12, \S 8.4 .5]$ for a discussion of this concept). Since $Q_{0}^{\mathrm{w}}$ is selfadjoint the uniqueness of microlocal solutions gives uniquess of eigenfunctions as they have to be orthogonal.

We have

$$
Q^{\mathrm{w}}=\left(\begin{array}{cc}
0 & \Lambda_{+}^{\mathrm{w}} \\
\Lambda_{-}^{\mathrm{w}} & 0
\end{array}\right), \quad \Lambda_{ \pm}(x, \xi):=\frac{1+e^{ \pm i x}+e^{ \pm i \xi}}{3}, \quad\left(\Lambda_{ \pm}^{\mathrm{w}}\right)^{*}=\Lambda_{\mp}^{\mathrm{w}} .
$$

Because of the symmetry $(x, \xi) \rightarrow(-x,-\xi)$ we will work microlocally near $\left(\frac{2 \pi}{3},-\frac{2 \pi}{3}\right)$. At that point (5.1) shows that the Poisson brackets of $\Lambda_{ \pm}$satisfy

$$
\left\{\operatorname{Re} \Lambda_{+}, \operatorname{Im} \Lambda_{+}\right\}<0, \quad\left\{\operatorname{Re} \Lambda_{-}, \operatorname{Im} \Lambda_{-}\right\}>0, \quad \frac{1}{i}\left\{\Lambda_{+}, \Lambda_{-}\right\}>0 .
$$

The last inequality is also known as Hörmander's hypoellipticity condition. Using $[\mathrm{Zw} 12, \S \S 12.4$ and 12.5$]$ we see that the first two inequalities in (5.10) show that there exist microlocally unique solutions

$$
\Lambda_{+}^{\mathrm{w}} u_{0}=\mathcal{O}\left(h^{\infty}\right), \quad \mathrm{WF}_{h}(u) \subset \operatorname{nbhd}\left(\left(\frac{2 \pi}{3},-\frac{2 \pi}{3}\right)\right) .
$$

On the other hand the last inequality in (5.10) shows that

$$
\mathrm{WF}_{h}(u) \subset \operatorname{nbhd}\left(\left(\frac{2 \pi}{3},-\frac{2 \pi}{3}\right)\right) \Longrightarrow\left\langle\Lambda_{+}^{\mathrm{w}} \Lambda_{-}^{\mathrm{w}} u, u\right\rangle \geq c_{0} h\|u\|^{2},
$$

see for instance the proof of [Zw12, Theorem 7.5]. This characterizes the microlocal kernel of $Q^{\mathrm{w}}$ near $\left(\frac{2 \pi}{3},-\frac{2 \pi}{3}\right)$. Since $\left(Q^{\mathrm{w}}\right)^{*} Q^{\mathrm{w}}=\operatorname{diag}\left(\Lambda_{-}^{\mathrm{w}} \Lambda_{+}^{\mathrm{w}}, \Lambda_{+}^{\mathrm{w}} \Lambda_{-}^{\mathrm{w}}\right)$, this means that all 
solutions to (5.9) other than the unique solution $\left(0, u_{0}\right)$ satisfy $|z| \geq c \sqrt{h}$. That gives the correspondence with microlocal solutions $w$ (satisfying (5.8)) to

$$
\begin{gathered}
H_{+} w=\lambda w, \quad \mathrm{WF}_{h}(w) \subset \operatorname{nbhd}\left(\left(\frac{2 \pi}{3},-\frac{2 \pi}{3}\right)\right), \quad H_{+}:=\Lambda_{+}^{\mathrm{w}} \Lambda_{-}^{\mathrm{w}} \\
\left(\begin{array}{cc}
0 & \Lambda_{+}^{\mathrm{w}} \\
\Lambda_{-}^{\mathrm{w}} & 0
\end{array}\right)\left(\begin{array}{l}
u_{1} \\
u_{2}
\end{array}\right)=z\left(\begin{array}{l}
u_{1} \\
u_{2}
\end{array}\right), \quad \mathrm{WF}_{h}\left(u_{j}\right) \subset \operatorname{nbhd}\left(\left(\frac{2 \pi}{3},-\frac{2 \pi}{3}\right)\right) \\
z= \pm \sqrt{\lambda}, \quad u_{1}=w, \quad u_{2}=z^{-1} \Lambda_{-}^{\mathrm{w}} w .
\end{gathered}
$$

Recalling (5.1) we see that $H_{+}$, microlocally near $\left(\frac{2 \pi}{3},-\frac{2 \pi}{3}\right)$ has the structure of a potential well and the distribution of eigenvalues near 0 has been extensively studied. Following earlier works of Weinstein [We77] and Colin de Verdière [CdV80] the semiclassical version was given by Helffer-Robert [HR84] and a clear outline can be found in [Sj89, §8, Case II, p.292]. In particular, there exists a function $F$ with an expansion $F(\omega, h) \sim F_{0}(\omega)+h F_{1}+h^{2} F_{2}(\omega) \cdots$, where $F_{1}$ is a constant (see [HR84, Corollaire (3.15)]) such that $\mathcal{O}\left(h^{\infty}\right)$ quasimodes of $H_{+}$are given by the quantization condition $F\left(\lambda_{n}(h), h\right)=n h, n=0,1, \cdots$. Since we have shown that $\lambda_{0}(h)=\mathcal{O}\left(h^{\infty}\right)$ we obtain that $F_{j}(0)=0$ for all $j$. That gives (5.6).

The spectrum and eigenfunctions of $Q_{0}^{\mathrm{w}}$ will now be used to describe $\left(Q^{\mathrm{w}}-z\right)^{-1}$ for $|\operatorname{Im} z|>h^{M}$ for any fixed $M$.

We first show that away from the spectrum of $Q_{0}^{\mathrm{w}}, Q^{\mathrm{w}}-z$ is invertible. The proof is a simpler version of the proof of Proposition 5.4 and the estimates are similar.

Lemma 5.2. Let $0<\delta_{1}<\delta_{0}$ and suppose that $z \in\left[-\delta_{1}, \delta_{1}\right]-i[-1,1]$ satisfies

$$
d\left(z, \operatorname{Spec}\left(Q_{0}^{\mathrm{w}}(x, h D)\right)\right)>h^{N_{0}},
$$

for some fixed $N_{0}$. Then for $0<h<h_{0}$,

$$
\left(Q^{\mathrm{w}}(x, h D)-z\right)^{-1}=\mathcal{O}\left(d\left(z, \operatorname{Spec}\left(Q_{0}^{\mathrm{w}}(x, h D)\right)\right)^{-1}\right)_{L^{2} \rightarrow L^{2}}
$$

Proof. In addition to $Q_{0}^{\mathrm{w}}$ we define another auxiliary operator with the symbol

$$
\begin{gathered}
Q_{1}(x, \xi):=Q_{0}(x, \xi)+\left(\begin{array}{cc}
-\chi_{1}(x, \xi) & 0 \\
0 & \chi_{1}(x, \xi)
\end{array}\right), \\
\chi_{1} \in C_{\mathrm{c}}^{\infty}\left(\mathbb{R}^{2} ;[0,1]\right), \quad \chi_{1}(\rho)=\chi_{1}(-\rho), \quad \chi_{1}(\rho)=\left\{\begin{array}{cc}
1, & \|\rho\|_{\infty}<\pi-\frac{2}{10}, \\
0, & \|\rho\|_{\infty}>\pi-\frac{1}{10},
\end{array}\right.
\end{gathered}
$$

noting that $Q_{1}(x, \xi)-z \in S(1)$ is now elliptic (in the sense that the determinant, $z^{2}-\chi_{1}^{2}+\operatorname{det} Q_{0}$, satisfies the conditions of [Zw12, §4.7.1] for $z$ in a neighbourhood of $0)$. From [Zw12, Theorems 4.29, 8.3] we conclude that

$$
\left(Q_{1}^{\mathrm{w}}(x, h D)-z\right)^{-1}=R_{1}^{\mathrm{w}}(z ; x, h D, h), \quad R_{1} \in S(1), \quad z \in\left[-\delta_{1}, \delta_{1}\right]-i[-1,1] .
$$


Using $Q_{0}^{\mathrm{w}}$ and $Q_{1}^{\mathrm{w}}$ we define

$$
\begin{aligned}
& p=p(z ; x, \xi):=Q(x, \xi)-z \\
& p_{0}^{\gamma}=p_{0}^{\gamma}(z ; x, \xi):=Q_{0}\left(x-\gamma_{1}, \xi-\gamma_{2}\right)-z \\
& p_{1}^{\gamma}=p_{1}^{\gamma}(z ; x, \xi):=Q_{1}\left(x-\gamma_{1}, \xi-\gamma_{2}\right)-z .
\end{aligned}
$$

We denote the Weyl quantizations by $P=P(z), P_{0}^{\gamma}=P_{0}^{\gamma}(z)$ and $P_{1}^{\gamma}=P_{1}^{\gamma}(z)$ and note that

$$
P_{0}^{\gamma}=r_{\gamma}\left(Q_{0}^{\mathrm{w}}-z\right) r_{-\gamma}, \quad P_{1}^{\gamma}=r_{\gamma}\left(Q_{1}^{\mathrm{w}}-z\right) r_{-\gamma}, \quad r_{\gamma} u(x):=e^{\frac{i}{h} \gamma_{2} x} u\left(x-\gamma_{1}\right) .
$$

We always assume that $z \in\left[-\delta_{1}, \delta_{1}\right]-i[-1,1]$.

We now choose $\chi, \tilde{\chi} \in C_{\mathrm{c}}^{\infty}\left(\mathbb{R}^{2}\right)$ so that

$$
\begin{gathered}
\left.\tilde{\chi}\right|_{\operatorname{nbhd}(\operatorname{supp} \chi)}=1,\left.\quad \chi_{0}\right|_{\operatorname{nbhd}(\operatorname{supp} \tilde{\chi})}=1, \\
\sum_{\gamma \in \mathbb{Z}_{*}^{2}} \chi_{\gamma}=1, \quad \chi_{\gamma}(x, \xi):=\chi\left(x-\gamma_{1}, \xi-\gamma_{2}\right) .
\end{gathered}
$$

We also define translations $\tilde{\chi}_{\gamma}(x, \xi):=\tilde{\chi}\left(x-\gamma_{1}, \xi-\gamma_{2}\right)$ and note that for all $N$ and with semi-norms independent of $\gamma$,

$$
\chi_{\gamma}, \tilde{\chi}_{\gamma} \in S\left(m_{\gamma}^{-N}\right), \quad m_{\gamma}(x, \xi):=\left(1+\left(x-\gamma_{1}\right)^{2}+\left(\xi-\gamma_{2}\right)^{2}\right)^{\frac{1}{2}}
$$

The properties of the cut-off functions guarantee that

$$
\left.\left(p-p_{0}^{\gamma}\right)\right|_{\operatorname{nbhd}\left(\operatorname{supp} \tilde{\chi}_{\gamma}\right)}=0,\left.\quad\left(p_{0}^{\gamma}-p_{1}^{\gamma}\right)\right|_{\operatorname{nbhd}\left(\operatorname{supp} \nabla \tilde{\chi}_{\gamma}\right)}=0 .
$$

Combined with (5.15) the composition formula for pseudodifferential operators [Zw12, Theorem 4.18] gives

$$
\begin{gathered}
e_{1, \gamma}^{\mathrm{w}}:=\left(P-P_{0}^{\gamma}\right) \tilde{\chi}_{\gamma}^{\mathrm{w}}, \quad e_{2, \gamma}^{\mathrm{w}}:=\tilde{\chi}_{\gamma}^{\mathrm{w}} \chi_{\gamma}^{\mathrm{w}}-\chi_{\gamma}^{\mathrm{w}}, \\
e_{3, \gamma}^{\mathrm{w}}:=\left[P_{0}^{\gamma}, \tilde{\chi}_{\gamma}^{\mathrm{w}}\right] \widetilde{P}_{\gamma}^{-1} \chi_{\gamma}^{\mathrm{w}}, \quad e_{4, \gamma}^{\mathrm{w}}:=\left[P_{0}^{\gamma}, \tilde{\chi}_{\gamma}^{\mathrm{w}}\right]\left(P_{0}^{\gamma}\right)^{-1}\left(P_{1}^{\gamma}-P_{0}^{\gamma}\right),
\end{gathered}
$$

where $e_{j, \gamma} \in h^{N} S\left(m_{\gamma}^{-N}\right)$, for all $N$.

If $d\left(z, \operatorname{Spec}\left(Q_{0}^{\mathrm{w}}\right)\right)>h^{N_{0}}$ we define $F^{0}:=\sum_{\gamma \in \mathbb{Z}_{*}^{2}} \tilde{\chi}_{\gamma}^{\mathrm{w}}\left(P_{0}^{\gamma}\right)^{-1} \chi_{\gamma}^{\mathrm{w}}$, where the inverse of $P_{0}^{\gamma}$ exists in view of (5.17). We claim that

$$
F^{0}:=\sum_{\gamma \in \mathbb{Z}_{*}^{2}} \tilde{\chi}_{\gamma}^{\mathrm{w}}\left(P_{0}^{\gamma}\right)^{-1} \chi_{\gamma}^{\mathrm{w}}=\mathcal{O}\left(d\left(z, \operatorname{Spec}\left(Q_{0}^{\mathrm{w}}\right)\right)^{-1}\right)_{L^{2} \rightarrow L^{2}}
$$

In fact, in view of (5.19)

$$
\tilde{\chi}_{\gamma}^{\mathrm{w}}\left(\tilde{\chi}_{\beta}^{\mathrm{w}}\right)^{*}=\left(a_{\gamma \beta}^{1}\right)^{\mathrm{w}}, \quad \chi_{\gamma}^{\mathrm{w}}\left(\chi_{\beta}^{\mathrm{w}}\right)^{*}=\left(a_{\gamma \beta}^{2}\right)^{\mathrm{w}}, \quad a_{\gamma \beta}^{j} \in S\left(m_{\gamma}^{-N} m_{\beta}^{-N}\right) .
$$

From [Zw12, Theorem 4.23]

$$
\left\|\left(a_{\gamma \beta}^{j}\right)^{\mathrm{w}}\right\|_{L^{2} \rightarrow L^{2}} \leq C \sup _{\mathbb{R}^{2}} m_{\gamma}^{-N} m_{\beta}^{-N} \leq C_{N}\langle\gamma-\beta\rangle^{-N},
$$


for all $N \in \mathbb{N}$. If we put $A_{\gamma}:=\tilde{\chi}_{\gamma}^{\mathrm{w}}\left(P_{0}^{\gamma}\right)^{-1} \chi_{\gamma}^{\mathrm{w}}$, if follows that

$$
A_{\gamma}^{*} A_{\beta}, A_{\gamma} A_{\beta}^{*}=\mathcal{O}\left(d\left(z, \operatorname{Spec}\left(Q_{0}^{\mathrm{w}}\right)\right)^{-2}\langle\gamma-\beta\rangle^{-N}\right)_{L^{2} \rightarrow L^{2}},
$$

and (5.22) follows from an application of the Cotlar-Stein Lemma - see [Zw12, Theorem C.5].

Using the notation of (5.21) we have

$$
\begin{aligned}
P F^{0}= & \sum_{\gamma \in \mathbb{Z}_{*}^{2}} P_{0}^{\gamma} \tilde{\chi}_{\gamma}^{\mathrm{w}}\left(P_{0}^{\gamma}\right)^{-1} \chi_{\gamma}^{\mathrm{w}}+e_{1, \gamma}\left(P_{0}^{\gamma}\right)^{-1} \chi_{\gamma}^{\mathrm{w}} \\
= & \sum_{\gamma \in \mathbb{Z}_{*}^{2}} \chi_{\gamma}^{\mathrm{w}}+e_{1, \gamma}^{\mathrm{w}}\left(P_{0}^{\gamma}\right)^{-1} \chi_{\gamma}^{\mathrm{w}}+e_{2, \gamma}^{\mathrm{w}}+\left[P_{0}^{\gamma}, \tilde{\chi}_{\gamma}^{\mathrm{w}}\right]\left(P_{0}^{\gamma}\right)^{-1} \chi_{\gamma}^{\mathrm{w}} \\
= & \mathrm{id}+\sum_{\gamma \in \mathbb{Z}_{*}^{2}} e_{1, \gamma}^{\mathrm{w}}\left(P_{0}^{\gamma}\right)^{-1} \chi_{\gamma}^{\mathrm{w}}+e_{2, \gamma}^{\mathrm{w}}+\left[P_{0}^{\gamma}, \tilde{\chi}_{\gamma}^{\mathrm{w}}\right]\left(P_{1}^{\gamma}\right)^{-1} \chi_{\gamma}^{\mathrm{w}} \\
& \quad+\sum_{\gamma \in \mathbb{Z}_{*}^{2}}\left[P_{0}^{\gamma}, \tilde{\chi}_{\gamma}^{\mathrm{w}}\right]\left(\left(P_{0}^{\gamma}\right)^{-1}-\left(P_{1}^{\gamma}\right)^{-1}\right) \chi_{\gamma}^{\mathrm{w}} \\
= & \mathrm{id}+\sum_{\gamma \in \mathbb{Z}_{*}^{2}} e_{1, \gamma}^{\mathrm{w}}\left(P_{1}^{\gamma}\right)^{-1} \chi_{\gamma}^{\mathrm{w}}+e_{2, \gamma}^{\mathrm{w}}+e_{3, \gamma}^{\mathrm{w}}+\left[P_{0}^{\gamma}, \tilde{\chi}_{\gamma}^{\mathrm{w}}\right]\left(P_{1}^{\gamma}\right)^{-1}\left(P_{1}^{\gamma}-P_{0}^{\gamma}\right)\left(P_{0}^{\gamma}\right)^{-1} \chi_{\gamma}^{\mathrm{w}} \\
= & \mathrm{id}+\sum_{\gamma \in \mathbb{Z}_{*}^{2}} e_{1, \gamma}^{\mathrm{w}}\left(P_{0}^{\gamma}\right)^{-1} \chi_{\gamma}^{\mathrm{w}}+e_{2, \gamma}^{\mathrm{w}}+e_{3, \gamma}^{\mathrm{w}}+e_{4, \gamma}^{\mathrm{w}}\left(P_{0}^{\gamma}\right)^{-1} \chi_{\gamma}^{\mathrm{w}} \\
= & \mathrm{id}+r, \quad r=\mathcal{O}\left(h^{\infty} d\left(z, \operatorname{Spec}\left(Q_{0}^{\mathrm{w}}\right)\right)^{-1}\right)_{L^{2} \rightarrow L^{2}},
\end{aligned}
$$

where the bound on $r$ follows from (5.21) and (5.24) and an application of the CotlarStein Lemma as in the proof of (5.22).

Hence for $h$ small enough,

$$
\left(Q^{\mathrm{w}}(x, h D)-z\right)^{-1}=F^{0}(\mathrm{id}+r)^{-1}=\mathcal{O}\left(d\left(z, \operatorname{Spec}\left(Q_{0}^{\mathrm{w}}\right)\right)^{-1}\right)_{L^{2} \rightarrow L^{2}}
$$

for $z \in\left[-\delta_{1}, \delta_{1}\right]-i[-1,1], d\left(z, \operatorname{Spec}\left(Q_{0}^{\mathrm{w}}\right)\right)>h^{N_{0}}$.

The proof gives a stronger weighted estimate on the inverse with similar estimates being crucial later. Under the assumption of Lemma 5.2 we have, for any $s \in \mathbb{R}$ and $G^{\mathrm{w}}$ defined in (5.4)

$$
e^{-s G^{\mathrm{w}}}\left(Q^{\mathrm{w}}-z\right)^{-1} e^{s G^{\mathrm{w}}}=\mathcal{O}\left(d\left(z, \operatorname{Spec}\left(Q_{0}^{\mathrm{w}}(x, h D)\right)\right)^{-1}\right)_{L^{2} \rightarrow L^{2}}
$$

Proof of (5.26). We first check that $F^{0}$ defined in (5.22) satisfies this estimate. (We note that (5.26) does not seem to follow easily from conjugating $Q^{\mathrm{w}}-z$ by the weight.) For that we make the following observations:

$$
\begin{gathered}
e^{s G^{\mathrm{w}}} \tilde{\chi}_{\gamma}^{\mathrm{w}}=\left(\tilde{\chi}_{\gamma}^{s}\right)^{\mathrm{w}}, \quad \chi_{\gamma}^{\mathrm{w}} e^{s G^{\mathrm{w}}}=\left(\chi_{\gamma}^{s}\right)^{\mathrm{w}}, \\
\tilde{\chi}_{\gamma}^{s}, \chi_{\gamma}^{s} \in \bigcap_{N} S\left(e^{s G} m_{\gamma}^{-N}\right)=\langle\gamma\rangle^{s} \bigcap_{N} S\left(m_{\gamma}^{-N}\right),
\end{gathered}
$$


where the equality of symbols spaces follows from the fact that $e^{s G(\rho)}=\langle\rho\rangle^{s}$ and

$$
\langle\rho\rangle^{s}\langle\rho-\gamma\rangle^{-N} \leq\langle\gamma\rangle^{s}\langle\rho-\gamma\rangle^{-N+|s|} \leq\langle\rho\rangle^{s}\langle\rho-\gamma\rangle^{-N+2|s|} .
$$

Proceeding as in (5.23) and (5.24) and putting $A_{\gamma}^{s}:=e^{-s G^{\mathrm{w}}} A_{\gamma} e^{s G^{\mathrm{w}}}$ we see that estimates (5.25) hold for $A_{\gamma}^{s}$. That shows that $e^{-s G^{\mathrm{w}}} F^{0} e^{s G^{\mathrm{w}}}$ is bounded on $L^{2}$ for any $s \in \mathbb{R}$. The same argument applies to $r$ in (5.26) and that concludes the proof of $(5.26)$.

We now use the translates of $w_{n}^{ \pm}$from Proposition 5.1 to construct a Grushin problem for $Q^{\mathrm{w}}-z$ for $z$ near $\left.\operatorname{Spec}\left(Q_{0}^{\mathrm{w}}\right)\right)$. For that we take $z_{1}$ and $\varepsilon_{0}$ such that

$$
\{\kappa(n h, h)\}_{n \in \mathbb{Z}} \cap\left[z_{1}-2 \varepsilon_{0} h, z_{1}+2 \varepsilon_{0} h\right]=\left\{\kappa\left(n_{1} h, h\right)\right\}, \quad n_{1}=n_{1}\left(z_{1}, h\right) .
$$

The interval $\left[-\delta_{0}, \delta_{0}\right]$ can be covered by intervals of this form and intervals of size $h$, disjoint from $\operatorname{Spec}\left(Q_{0}^{\mathrm{w}}\right)$.

For $\gamma \in \mathbb{Z}_{*}^{2}$ we use translation (5.17) and put

$$
w_{\gamma}=w_{\gamma}(h):=\left(w_{\gamma}^{+}(h), w_{\gamma}^{-}(h)\right)=\left(r_{\gamma} u_{n_{1}}^{+}(h), r_{\gamma} u_{n_{1}}^{-}(h)\right) \in \mathbb{C}^{2} \otimes \mathbb{C}^{2},
$$

where $n_{1}$ is defined by (5.29).

The following lemma will be useful in several places:

Lemma 5.3. With $w_{\gamma}^{ \pm}$defined by (5.30) and $G$ given in (5.4) we have, for every $s \in \mathbb{R}$,

$$
\begin{aligned}
\left\langle e^{s G^{\mathrm{w}}} w_{\gamma}^{ \pm}, e^{s G^{\mathrm{w}}} w_{\beta}^{ \pm}\right\rangle & =\mathcal{O}\left(\langle\gamma\rangle^{2 s} \delta_{\gamma \beta}+h^{\infty}\langle\gamma\rangle^{2 s}\langle\gamma-\beta\rangle^{-\infty}\right), \\
\left\langle e^{s G^{\mathrm{w}}} w_{\gamma}^{+}, e^{s G^{\mathrm{w}}} w_{\beta}^{-}\right\rangle & =\mathcal{O}\left(h^{\infty}\langle\gamma\rangle^{2 s}\langle\gamma-\beta\rangle^{-\infty}\right), \\
\left\langle e^{s G^{\mathrm{w}}}\left(1-\chi_{\gamma}^{\mathrm{w}}\right) w_{\gamma}^{\varepsilon}, e^{s G^{\mathrm{w}}}\left(1-\chi_{\beta}^{\mathrm{w}}\right) w_{\beta}^{\varepsilon^{\prime}}\right\rangle & =\mathcal{O}\left(h^{\infty}\langle\gamma\rangle^{2 s}\langle\gamma-\beta\rangle^{-\infty}\right), \quad \varepsilon, \varepsilon^{\prime} \in\{+,-\} .
\end{aligned}
$$

Proof. This follows from (5.7), (5.8) and arguments presented in the remark above. As an example we prove the first estimate in (5.31) (dropping \pm in the notation):

$$
\begin{aligned}
\left\langle e^{s G^{\mathrm{w}}} w_{\gamma}, e^{s G^{\mathrm{w}}} w_{\beta}\right\rangle= & \left\langle e^{s G^{\mathrm{w}}}\left(1-\chi_{\gamma}^{\mathrm{w}}\right) w_{\gamma}, e^{s G^{\mathrm{w}}}\left(1-\chi_{\beta}^{\mathrm{w}}\right) w_{\beta}\right\rangle+\left\langle e^{s G^{\mathrm{w}}} \chi_{\gamma}^{\mathrm{w}} w_{\gamma}, e^{s G^{\mathrm{w}}} \chi_{\beta}^{\mathrm{w}} w_{\beta}\right\rangle \\
& +\left\langle e^{s G^{\mathrm{w}}} \chi_{\gamma}^{\mathrm{w}} w_{\gamma}, e^{s G^{\mathrm{w}}}\left(1-\chi_{\beta}^{\mathrm{w}}\right) w_{\beta}\right\rangle+\left\langle e^{s G^{\mathrm{w}}}\left(1-\chi_{\gamma}^{\mathrm{w}}\right) w_{\gamma}, e^{s G^{\mathrm{w}}} \chi_{\beta}^{\mathrm{w}} w_{\beta}\right\rangle
\end{aligned}
$$

With $G_{\gamma}(\rho):=G(\rho-\gamma)$,

$$
e^{s G^{\mathrm{w}}-N G_{\gamma}^{\mathrm{w}}} e^{N G_{\gamma}^{\mathrm{w}}}\left(1-\chi_{\gamma}^{\mathrm{w}}\right) w_{\gamma}=b_{\gamma}^{\mathrm{w}}(x, h D) e^{N G_{\gamma}^{\mathrm{w}}}\left(1-\chi_{\gamma}^{\mathrm{w}}\right) w_{\gamma},
$$

where as in (5.28),

$$
b_{\gamma} \in S\left(\langle\rho\rangle^{s}\langle\rho-\gamma\rangle^{-N}\right) \subset S\left(\langle\gamma\rangle^{s}\langle\rho-\gamma\rangle^{-N+|s|}\right) .
$$

Putting

$$
\tilde{w}_{\gamma}:=e^{N G_{\gamma}^{\mathrm{w}}}\left(1-\chi_{\gamma}^{\mathrm{w}}\right) w_{\gamma}=\mathcal{O}\left(h^{\infty}\right)_{L^{2}}
$$


and $M=N-|s| \gg 1$, we see that

$$
\begin{aligned}
\left\langle e^{s G^{\mathrm{w}}}\left(1-\chi_{\gamma}^{\mathrm{w}}\right) w_{\gamma}, e^{s G^{\mathrm{w}}}\left(1-\chi_{\beta}^{\mathrm{w}}\right) w_{\beta}\right\rangle & =\left\langle\left(b_{\beta}^{\mathrm{w}}\right)^{*} b_{\gamma}^{\mathrm{w}} \tilde{w}_{\gamma}, \tilde{w}_{\beta}\right\rangle=\left\|\left(b_{\beta}^{\mathrm{w}}\right)^{*} b_{\gamma}^{\mathrm{w}}\right\|_{L^{2} \rightarrow L^{2}} \mathcal{O}\left(h^{\infty}\right) \\
& \leq C \sup _{\rho \in \mathbb{R}^{2}}\langle\gamma\rangle^{s}\langle\beta\rangle^{s}\langle\rho-\gamma\rangle^{-M}\langle\rho-\beta\rangle^{-M} \mathcal{O}\left(h^{\infty}\right) \\
& \leq \mathcal{O}\left(h^{\infty}\langle\gamma\rangle^{2 s}\langle\gamma-\beta\rangle^{-M+s}\right) .
\end{aligned}
$$

The other terms are treated in the same way.

We then define $R_{+}: L^{2}\left(\mathbb{R}, \mathbb{C}^{2}\right) \rightarrow \ell^{2}\left(\mathbb{Z}_{*}^{2} ; \mathbb{C}^{2}\right)$ and $R_{-}=\ell^{2}\left(\mathbb{Z}_{*}^{2} ; \mathbb{C}^{2}\right) \rightarrow L^{2}\left(\mathbb{R}, \mathbb{C}^{2}\right)$ as follows

$$
\left(R_{+} u\right)(\gamma):=\left\langle u, w_{\gamma}\right\rangle:=\left(\begin{array}{l}
\left\langle u, w_{\gamma}^{+}\right\rangle \\
\left\langle u, w_{\gamma}^{-}\right\rangle
\end{array}\right) \in \mathbb{C}^{2}, \quad R_{-} u_{-}(x):=\sum_{\gamma \in \mathbb{Z}_{*}^{2}} w_{\gamma}(x) u_{-}(\gamma),
$$

where $u_{-}(\gamma)=\left(u_{-}^{+}(\gamma), u_{-}^{-}(\gamma)\right)^{t} \in \mathbb{C}^{2}$ and $w_{\gamma}(x)=\left(w_{\gamma}^{+}, w_{\gamma}^{-}\right) \in \mathbb{C}^{2} \otimes \mathbb{C}^{2}$.

To see the boundedness of $R_{-}$we use the almost orthogonality of $w_{\gamma}^{ \pm}$given in (5.31) with $s=0$ : Hence,

$$
\begin{aligned}
\left\|\sum_{\gamma \in \mathbb{Z}_{*}} w_{\gamma}(\bullet) u_{-}(\gamma)\right\|_{L^{2}}^{2} & \lesssim \sum_{\gamma \in \mathbb{Z}_{*}} \sum_{\beta \in \mathbb{Z}_{*}}\left|u_{-}(\gamma) \| u_{-}(\gamma+\beta)\right|\langle\beta\rangle^{-N} \\
& \lesssim\left\|u_{-}\right\|_{\ell^{2}}\left(\sum_{\gamma \in \mathbb{Z}_{*}}\left(\sum_{\gamma \in \mathbb{Z}_{*}}\left|u_{-}(\gamma+\gamma)\right|\langle\gamma\rangle^{-N}\right)^{2}\right)^{\frac{1}{2}} \\
& \lesssim\left\|u_{-}\right\|_{\ell^{2}}\left(\sum_{\gamma \in \mathbb{Z}_{*}} \sum_{\gamma \in \mathbb{Z}_{*}} \sum_{\gamma^{\prime} \in \mathbb{Z}_{*}}\left|u_{-}(\gamma+\gamma)\right|^{2}\langle\gamma\rangle^{-N}\left\langle\gamma^{\prime}\right\rangle^{-N}\right)^{\frac{1}{2}} \\
& \lesssim\left\|u_{-}\right\|_{\ell^{2}}^{2} .
\end{aligned}
$$

(This is a version of Schur's argument, see for instance [Zw12, Proof of Theorem 4.21, Step 2,]; later on we will again need the Cotlar-Stein Lemma as in the proof of boundedness of $F^{0}$ in the proof of Lemma 5.2.) Since $R_{+}=R_{-}^{*}$ the boundedness of $R_{+}$also follows. We note that $R_{+} R_{-}=\operatorname{id}_{\ell^{2}\left(\mathbb{Z}_{*}^{2} ; \mathbb{C}^{2}\right)}$.

Proposition 5.4. Assume that (5.29) holds and that $R_{ \pm}$are defined by (5.32). Then the Grushin problem

$$
\left(\begin{array}{cc}
Q^{\mathrm{w}}(x, h D)-z & R_{-} \\
R_{+} & 0
\end{array}\right): L^{2}\left(\mathbb{R}, \mathbb{C}^{2}\right) \times \ell^{2}\left(\mathbb{Z}_{*}^{2} ; \mathbb{C}^{2}\right) \longrightarrow L^{2}\left(\mathbb{R}, \mathbb{C}^{2}\right) \times \ell^{2}\left(\mathbb{Z}_{*}^{2} ; \mathbb{C}^{2}\right),
$$

is well posed for $z \in\left(z_{1}-\varepsilon_{0} h, z_{1}+\varepsilon_{0} h\right)+i(-1,1)$, with the inverse

$$
\left(\begin{array}{cc}
E(z, h) & E_{+}(z, h) \\
E_{-}(z, h) & E_{-+}(z, h)
\end{array}\right)=\left(\begin{array}{cc}
\mathcal{O}(1 / h)_{L^{2} \rightarrow L^{2}} & \mathcal{O}(1)_{\ell^{2} \rightarrow L^{2}} \\
\mathcal{O}(1)_{L^{2} \rightarrow \ell^{2}} & \mathcal{O}(h)_{\ell^{2} \rightarrow \ell^{2}}
\end{array}\right) .
$$


In addition,

$$
\begin{gathered}
\left(E_{-+}(z, h) v_{+}\right)(\gamma)=\sum_{\beta \in \mathbb{Z}_{*}^{2}} E_{-+}(\gamma-\beta) v_{+}(\beta), \\
E_{-+}(\gamma)=\delta_{\gamma 0}\left(z-\kappa\left(n_{1} h, h\right)\right) \operatorname{id}_{\mathbb{C}^{2}}+\mathcal{O}\left(h^{\infty}\langle\gamma\rangle^{-\infty}\right)
\end{gathered}
$$

where $\kappa$ is given by (5.5) and $n_{1}$ by (5.29).

Before proceeding with the proof of Proposition 5.4 we explain the basic idea in a simple example. Suppose $P$ is a self-adjoint operator on a Hilbert space $H$, say a matrix, with $\operatorname{Spec}(P) \cap[-\delta, \delta]=\{0\}$, where 0 is a simple eigenvalue, $P w=0,\|w\|=1$. Then for $z \in([-\delta, \delta]+i \mathbb{R}) \backslash\{0\}$,

$$
(P-z)^{-1}=-\frac{w\langle\bullet, w\rangle}{z}+S(z), \quad(P-z) S(z)=\mathrm{id}-w\langle\bullet, w\rangle,
$$

and $S(z)$ is holomorphic.

We then define $R_{-}: \mathbb{C} \rightarrow H, R_{+}: H \rightarrow \mathbb{C}: R_{-} u_{-}=u_{-} w, R_{+} u=\langle u, w\rangle$. One easily checks $z \in[-\delta, \delta]+i \mathbb{R}$,

$$
\left(\begin{array}{cc}
P-z & R_{-} \\
R_{+} & 0
\end{array}\right)^{-1}=\left(\begin{array}{cc}
S(z) & R_{-} \\
R_{+} & z
\end{array}\right)=:\left(\begin{array}{cc}
E(z) & E_{+}(z) \\
E_{-}(z) & E_{-+}(z)
\end{array}\right): H \times \mathbb{C} \rightarrow H \times \mathbb{C} .
$$

We now follow a similar procedure for $P=Q^{\mathrm{w}}-z$ using approximate eigenfunctions $w_{\gamma}$ and a partition of $\left\{\chi_{\gamma}\right\}_{\gamma \in \mathbb{Z}_{*}^{2}}$ as in (5.22). The approximate inverse (5.41) is then similar to (5.37). To obtain the localization result in (5.36) we upgrade $L^{2} \times \ell^{2}$ estimates to weighted estimates (5.42) and (5.43), as in the remark after the proof of Lemma 5.2.

We also record translation symmetries of our Grushin problem:

Lemma 5.5. Suppose that $\gamma \in \mathbb{Z}_{*}^{2}, r_{\gamma}: L^{2}\left(\mathbb{R}^{2}\right) \rightarrow L^{2}\left(\mathbb{R}^{2}\right)$ is defined by (5.17) and $s_{\gamma}: \ell^{2}\left(\mathbb{Z}_{*}^{2}\right) \rightarrow \ell^{2}\left(\mathbb{Z}_{*}^{2}\right)$ by $\left(s_{\gamma} f\right)(\delta):=f(\delta-\gamma)$. Then in the notation of (5.34),

$$
\left(\begin{array}{cc}
r_{\gamma} & 0 \\
0 & s_{\gamma}
\end{array}\right)\left(\begin{array}{cc}
Q^{\mathrm{w}}(x, h D)-z & R_{-} \\
R_{+} & 0
\end{array}\right)=\left(\begin{array}{cc}
Q^{\mathrm{w}}(x, h D)-z & R_{-} \\
R_{+} & 0
\end{array}\right)\left(\begin{array}{cc}
r_{\gamma} & 0 \\
0 & s_{\gamma}
\end{array}\right), \quad \gamma \in \mathbb{Z}_{*}^{2} .
$$

Proof of Proposition 5.4. We follow the same procedure as in the proof of Lemma 5.2 and we use the notation from there.

To start we note that in our range of $z$ 's with $\kappa\left(n_{1} h, n_{1}\right)$ excluded,

$$
P_{\gamma}^{-1}=\frac{w_{\gamma}\left\langle\bullet, w_{\gamma}\right\rangle}{\kappa\left(n_{1} h, h\right)-z}+S_{\gamma}, \quad P_{\gamma} S_{\gamma}=\mathrm{id}-w_{\gamma}\left\langle\bullet, w_{\gamma}\right\rangle, \quad S_{\gamma}=\mathcal{O}(1 / h)_{L^{2} \rightarrow L^{2}},
$$

where the estimate on $S_{\gamma}$ follows from the holomorphy of $S_{\gamma}$ and the maximum principle: we can find $\varepsilon_{1}>\varepsilon_{0}$ such that on the boundary of $\left(z_{1}-\varepsilon_{1} h, z_{1}+\varepsilon_{1} h\right)+i(-2,2)$, $\left\|P_{\gamma}^{-1}\right\|=1 / d\left(z, \operatorname{Spec}\left(P_{\gamma}\right)\right)=\mathcal{O}(1 / h)$ and $\left|\kappa\left(n_{1} h, h\right)-z\right|^{-1}=\mathcal{O}(1 / h)$. 
For future reference will also note that

$$
S_{\gamma} u=\widetilde{P}_{\gamma}^{-1}\left(u-w_{\gamma}\left\langle u, w_{\gamma}\right\rangle\right)+\widetilde{P}_{\gamma}^{-1}\left(\widetilde{P}_{\gamma}-P_{\gamma}\right) S_{\gamma} u .
$$

In the notation of (5.22) and (5.39) we define $E_{\bullet}^{0}=E_{\bullet}^{0}(z)$ :

$$
\begin{gathered}
E^{0}:=\sum_{\gamma \in \mathbb{Z}_{*}^{2}} \widetilde{\chi}_{\gamma}^{\mathrm{w}} S_{\gamma} \chi_{\gamma}^{\mathrm{w}}, \\
E_{+}^{0}:=R_{-}, \quad E_{-}^{0}:=R_{+}, \quad E_{-+}^{0}=\left(z-\kappa\left(h n_{1}, n_{1}\right)\right) \mathrm{id}_{\ell^{2}} .
\end{gathered}
$$

Lemma 5.5 shows that $r_{\gamma} E_{+}^{0}=E_{+}^{0} T_{\gamma}$ and $E_{-}^{0} r_{\gamma}=T_{\gamma} E_{-}^{0}$. We now check that $r_{\gamma} E^{0} r_{-\gamma}=$ $E^{0}$. In fact, (5.17) shows that

$$
r_{\gamma} E^{0} r_{-\gamma}=\sum_{\gamma \in \mathbb{Z}_{*}^{2}} r_{\gamma} \widetilde{\chi}_{\gamma}^{\mathrm{w}} S_{\gamma} \chi_{\gamma}^{\mathrm{w}} r_{-\gamma} v=\sum_{\gamma \in \mathbb{Z}_{*}^{2}} \widetilde{\chi}_{\gamma+\gamma}^{\mathrm{w}} S_{\gamma+\gamma} \chi_{\gamma+\gamma}^{\mathrm{w}} v=E^{0} v .
$$

As in the proof of (5.26) we also see that for $G$ given by (5.4) and

$$
\begin{gathered}
(g u)(\gamma):=\log \langle\gamma\rangle u(\gamma), \\
\left(\begin{array}{cc}
e^{-s G^{\mathrm{w}}} & 0 \\
0 & e^{-s g}
\end{array}\right)\left(\begin{array}{cc}
E^{0} & E_{+}^{0} \\
E_{-}^{0} & E_{-+}^{0}
\end{array}\right)\left(\begin{array}{cc}
e^{s G^{\mathrm{w}}} & 0 \\
0 & e^{s g}
\end{array}\right)=\left(\begin{array}{cc}
\mathcal{O}(1 / h)_{L^{2} \rightarrow L^{2}} & \mathcal{O}(1)_{\ell^{2} \rightarrow L^{2}} \\
\mathcal{O}(1)_{L^{2} \rightarrow \ell^{2}} & \mathcal{O}(h)_{\ell^{2} \rightarrow \ell^{2}}
\end{array}\right) .
\end{gathered}
$$

We claim that

$$
\left(\begin{array}{cc}
Q^{\mathrm{w}}-z & R_{-} \\
R_{+} & 0
\end{array}\right)\left(\begin{array}{cc}
E^{0} & E_{+}^{0} \\
E_{-}^{0} & E_{-+}^{0}
\end{array}\right)=\mathrm{id}_{L^{2} \times \ell^{2}}+\left(\begin{array}{cc}
r & r_{+} \\
r_{-} & 0
\end{array}\right),
$$

where for all $s \in \mathbb{R}$,

$$
\left(\begin{array}{cc}
e^{-s G^{\mathrm{w}}} & 0 \\
0 & e^{-s g}
\end{array}\right)\left(\begin{array}{cc}
r & r_{+} \\
r_{-} & 0
\end{array}\right)\left(\begin{array}{cc}
e^{s G^{\mathrm{w}}} & 0 \\
0 & e^{s g}
\end{array}\right)=\mathcal{O}\left(h^{\infty}\right)_{L^{2} \times \ell^{2} \rightarrow L^{2} \times \ell^{2}}
$$

As in (5.26) (with (5.40) used to pass from the third line to the fourth line in (5.26)) and the proof of (5.26) we see that

$$
\begin{aligned}
P E^{0} v+R_{-} E_{-}^{0} v & =\sum_{\gamma} P \tilde{\chi}_{\gamma}^{\mathrm{w}} S_{\gamma} \chi_{\gamma}^{\mathrm{w}} v+w_{\gamma}\left\langle v, w_{\gamma}\right\rangle \\
& =v+\sum_{\gamma} w_{\gamma}\left(\left\langle v, w_{\gamma}\right\rangle-\left\langle v, \chi_{\gamma}^{\mathrm{w}} w_{\gamma}\right\rangle\right)+r_{1} v \\
& =\left(\mathrm{id}+r_{1}+r_{2}\right) v, \quad r_{2}:=\sum_{\gamma} w_{\gamma} \otimes\left(1-\chi_{\gamma}^{\mathrm{w}}\right) \bar{w}_{\gamma} .
\end{aligned}
$$

where $e^{-s G^{\mathrm{w}}} r_{1} e^{s G^{\mathrm{w}}}=\mathcal{O}\left(h^{\infty}\right)_{L^{2} \rightarrow L^{2}}$. To show that $e^{-s G^{\mathrm{w}}} r_{2} e^{s G^{\mathrm{w}}}=\mathcal{O}\left(h^{\infty}\right)_{L^{2} \rightarrow L^{2}}$ we use (5.31) and the bound follows again from the Cotlar-Stein Lemma (or from a direct estimate). 
The other estimates in (5.43) are proved similarly using the localization properties of $w_{\gamma}$. We start with $r_{+} v_{+}=P E_{+}^{0} v_{+}+E_{-+} v_{+}=\sum_{\gamma}\left(P-P_{\gamma}\right) w_{\gamma} v_{+}(\gamma)$. Hence,

$$
\begin{aligned}
\left(e^{-s G^{\mathrm{w}}} r_{+} e^{s g}\right) v_{+} & =\sum_{\gamma}\langle\gamma\rangle^{s} e^{-s G^{\mathrm{w}}} e^{-N r_{\gamma} G^{\mathrm{w}} r_{-\gamma}} r_{\gamma}\left(\left(P-P_{0}\right) e^{N G^{\mathrm{w}}} w_{0}\right) v_{+}(\gamma) \\
& =\sum_{\gamma} c_{\gamma}^{\mathrm{w}} u_{\gamma} v_{+}(\gamma), \quad c_{\gamma} \in S(1), \quad u_{\gamma}:=r_{\gamma}\left(\left(P-P_{0}\right) e^{N G^{\mathrm{w}}} w_{0}\right)
\end{aligned}
$$

As in the proof of Lemma $5.3\left\langle u_{\gamma}, u_{\beta}\right\rangle=\mathcal{O}\left(h^{\infty}\langle\gamma-\beta\rangle^{-\infty}\right)$ and from this the bound $e^{-s G^{\mathrm{w}}} r_{+} e^{s g}=\mathcal{O}\left(h^{\infty}\right)$ easily follows (see (5.33) for a similar argument).

For $r_{-}$we write

$$
\begin{aligned}
\langle\gamma\rangle^{-s}\left(r_{-} e^{s G^{\mathrm{w}}} v\right)(\gamma) & =\langle\gamma\rangle^{-s}\left(R_{+}\left(E_{-}^{0} e^{s G^{\mathrm{w}}} v\right)\right)(\gamma)=\left\langle v, \tilde{u}_{\gamma}\right\rangle \\
\tilde{u}_{\gamma} & :=\sum_{\gamma} \chi_{\gamma}^{\mathrm{w}} S_{\gamma}^{*} \tilde{\chi}_{\gamma}^{\mathrm{w}}\langle\gamma\rangle^{-s} w_{\gamma}
\end{aligned}
$$

We claim that $\left\langle\tilde{u}_{\gamma}, \tilde{u}_{\beta}\right\rangle=\mathcal{O}\left(h^{\infty}\langle\beta-\gamma\rangle^{-\infty}\right)$. This follows similarly to previous arguments using $\tilde{\chi}_{\gamma}^{\mathrm{w}} w_{\gamma}=\mathcal{O}\left(h^{\infty}|\gamma-\gamma|^{-\infty}\right), \gamma \neq \gamma$, and $S_{\gamma}^{*} w_{\gamma}=0$.

This concludes the proof of (5.43) and in turn that estimate shows that

$$
\left(\begin{array}{cc}
Q^{\mathrm{w}}-z & R_{-} \\
R_{+} & 0
\end{array}\right)^{-1}=\left(\begin{array}{cc}
E^{0} & E_{+}^{0} \\
E_{-}^{0} & E_{-+}^{0}
\end{array}\right)\left(\operatorname{id}_{L^{2} \times \ell^{2}}+\left(\begin{array}{cc}
\tilde{r} & \tilde{r}_{+} \\
\tilde{r}_{-} & \tilde{r}_{-+}
\end{array}\right)\right),
$$

where

$$
\left(\begin{array}{cc}
e^{-s G^{\mathrm{w}}} & 0 \\
0 & e^{-s g}
\end{array}\right)\left(\begin{array}{cc}
\tilde{r} & \tilde{r}_{+} \\
\tilde{r}_{-} & \tilde{r}_{-+}
\end{array}\right)\left(\begin{array}{cc}
e^{s G^{\mathrm{w}}} & 0 \\
0 & e^{s g}
\end{array}\right)=\mathcal{O}\left(h^{\infty}\right)_{L^{2} \times \ell^{2} \rightarrow L^{2} \times \ell^{2}}
$$

This and Lemma 5.5 imply (5.35) and (5.36).

\section{Density of states}

We now use the analysis of $\S 5$ to describe the renormalized trace of the resolvent of $Q^{\mathrm{w}}(x, h D)$. This will lead us to an explicit semiclassical description of the density of states of the Hamiltonian $H^{B}$ stated in (4.33).

The Schur complement formula and (5.35) gives for $\left|z-z_{1}\right| \leq \varepsilon_{0} h$,

$$
\left(Q^{\mathrm{w}}(x, h D)-z\right)^{-1}=E(z, h)-E_{+}(z, h) E_{-+}(z, h)^{-1} E_{-}(z, h) .
$$

Hence, by (4.3),

$$
\widehat{\operatorname{tr}}\left(Q^{\mathrm{w}}(x, h D)-z\right)^{-1}=G_{z_{1}}(z, h)+J_{z_{1}}(z, h)
$$

where

$$
G_{z_{1}}(z, h):=\frac{1}{4 \pi^{2}} \int_{\mathbb{T}_{*}^{2}} \operatorname{tr}_{\mathbb{C}^{2}} \sigma(E(z, h)) d x d \xi
$$


is holomorphic in $\left(z_{1}-\varepsilon_{0} h, z_{1}+\varepsilon_{0} h\right)+i(-1,1)$ and

$$
J_{z_{1}}(z, h):=\frac{1}{4 \pi^{2}} \int_{\mathbb{T}_{*}^{2}} \operatorname{tr}_{\mathbb{C}^{2}} \sigma\left(E_{+}(z, h) E_{-+}(z, h)^{-1} E_{-}(z, h)\right) d x d \xi .
$$

Dropping $(z, h)$ and writing $A:=E_{+} E_{-+}^{-1} E_{-}$, we are seeking $\sigma(A)$ for the operator with the Schwartz kernel, $K_{A}$, given by

$$
K_{A}(x, y)=\sum_{\gamma, \beta \in \mathbb{Z}_{*}^{2}} E_{+}(x, \gamma) E_{ \pm}^{-1}(\gamma-\beta) E_{-}(\beta, y)
$$

From (5.36) we see that

$$
\begin{aligned}
E_{-+}(\gamma) & =\delta_{\gamma 0} E_{-+}^{0}(\gamma)+\mathcal{O}\left(h^{\infty}\langle\gamma\rangle^{-\infty}\right) \\
& =\delta_{\gamma 0}\left(z-\kappa\left(n_{1} h, h\right)\right) \operatorname{id}_{\mathbb{C}^{2}}+\mathcal{O}\left(|\operatorname{Im} z|^{-1} h^{\infty}\langle\gamma\rangle^{-\infty}\right) .
\end{aligned}
$$

We recall that $z \in\left(z_{1}-\varepsilon_{0} h, z_{1}+\varepsilon_{0} h\right)+i(-1,1), n=n_{1}\left(z_{1}, h\right)$, and that (5.29) holds. It follows that for

$$
|\operatorname{Im} z|>h^{M}
$$

where $M$ is arbitrary and fixed, we have

$$
E_{-+}^{-1}(\gamma)=\left(z-\kappa\left(n_{1} h, h\right)\right)^{-1} \delta_{\gamma, 0} \operatorname{id}_{\mathbb{C}^{2}}+\mathcal{O}\left(h^{\infty}\langle\gamma\rangle^{-\infty}\right)
$$

We now want to use this expression of $E_{-+}^{-1}$ to analyse the symbol of $A$.

The leading term. To obtain the leading term in (6.2) we define

$$
J_{z_{1}}^{0}(z, h):=\frac{1}{4 \pi^{2}} \int_{\mathbb{T}_{*}^{2}}\left(z-\kappa\left(n_{1} h, h\right)\right)^{-1} \operatorname{tr}_{\mathbb{C}^{2}} \sigma\left(E_{+}^{0}(z, h) E_{-}^{0}(z, h)\right) d x d \xi,
$$

where the approximations of $E_{ \pm}, E_{ \pm}^{0}$, are defined in (5.41):

$$
\begin{gathered}
E_{+}^{0}(z, h) v_{+}(x)=\sum_{\gamma} w_{\gamma}(x) v_{+}(\gamma), \quad\left(E_{-}^{0}(z, h) v\right)(\gamma)=\left\langle v, w_{\gamma}\right\rangle=\left(\begin{array}{c}
\left\langle v, w_{\gamma}^{+}\right\rangle \\
\left\langle v, w_{\gamma}^{-}\right\rangle
\end{array}\right), \\
w_{\gamma}=\left(w_{\gamma}^{+}, w_{\gamma}^{-}\right)=\left(r_{\gamma} u_{n_{1}}^{+}(h), r_{\gamma} u_{n_{1}}^{-}\right), \quad v_{+} \in \ell^{2}\left(\mathbb{Z}_{*}^{2}, \mathbb{C}^{2}\right), \quad v \in L^{2}\left(\mathbb{R}, \mathbb{C}^{2}\right) .
\end{gathered}
$$

The inverse $E_{-+}^{-1}$ was replaced by the first term on the right hand side of (6.6).

To analyse $J_{z_{1}}^{0}$ we use the formula for the Weyl symbol in terms of the Schwartz kernel:

$$
\begin{gathered}
A u(x)=\int_{\mathbb{R}^{2}} K(x, y) u(y) d y, \quad K(x, y)=\frac{1}{2 \pi h} \int_{\mathbb{R}} a\left(\frac{x+y}{2}, \xi\right) e^{\frac{i}{h}(x-y)} d \xi \\
a(x, \xi)=\int K\left(x-\frac{w}{2}, x+\frac{w}{2}\right) e^{\frac{i}{h} w \xi} d w
\end{gathered}
$$


see $[\mathrm{Zw} 12, \oint 4.1]$. In our case $A=E_{+}^{0}(z, h) E_{-}^{0}(z, h)$, where (see $\left.(5.41)\right) E_{+}^{0}=R_{-}$, $E_{-}^{0}=R_{+}=R_{-}^{*}$, where $R_{ \pm}$are given in (5.32). That is,

$$
E_{+}^{0} f(x)=\sum_{\alpha} E_{+}^{0}(x, \alpha) f(\alpha), \quad E_{+}^{0}(x, \alpha)=w_{\alpha}(x)=\left(w_{\alpha}^{+}(x), w_{\alpha}^{-}(x)\right) \in \mathbb{C}^{2} \otimes \mathbb{C}^{2},
$$

where $f(\alpha)=\left(f^{+}(\alpha), f^{-}(\alpha)\right)^{t} \in \mathbb{C}^{2}$, and

$$
E_{-}^{0} u(\gamma)=\int_{\mathbb{R}} E_{-}(\gamma, x) u(x) d x, \quad E_{-}^{0}(\gamma, x)=w_{\gamma}(x)^{*}=\left(\begin{array}{c}
\bar{w}_{\gamma}^{+}(x) \\
\bar{w}_{\gamma}^{-}(x)
\end{array}\right), \quad u \in L^{2}\left(\mathbb{R} ; \mathbb{C}^{2}\right) .
$$

This means that

$$
K(x, y)=\sum_{\alpha} E_{+}^{0}(x, \alpha) E_{-}^{0}(\alpha, h)=\sum_{\alpha} w_{\alpha}(x) w_{\alpha}(y)^{*},
$$

which in turn gives,

$$
\begin{aligned}
\sigma\left(E_{+}^{0}(z, h) E_{-}^{0}(z, h)\right)(x, \xi) & =\sum_{\alpha} \int_{\mathbb{R}} w_{\alpha}\left(x-\frac{w}{2}\right) w_{\alpha}^{*}\left(x-\frac{w}{2}\right) e^{\frac{i}{h} w \xi} w \\
& =\sum_{\alpha} \int_{\mathbb{R}} e^{\frac{i}{h} w\left(\xi-\alpha_{2}\right)} w_{0}\left(x-\frac{w}{2}-\alpha_{1}\right) w_{0}\left(x+\frac{w}{2}-\alpha_{1}\right)^{*} d w .
\end{aligned}
$$

Hence,

$$
\begin{aligned}
& \int_{\mathbb{T}_{*}^{2}} \sigma\left(E_{+}^{0}(z, h) E_{-}^{0}(z, h)\right) \frac{d x d \xi}{4 \pi^{2}}= \\
& \sum_{\alpha} \int_{\mathbb{T}_{*}^{2}} \int_{\mathbb{R}} e^{\frac{i}{h} w\left(\xi-\alpha_{2}\right)} w_{0}\left(x-\frac{w}{2}-\alpha_{1}\right) w_{0}\left(x+\frac{w}{2}-\alpha_{1}\right)^{*} d w \frac{d x d \xi}{4 \pi^{2}}= \\
& \quad \int_{\mathbb{R}^{2}} \int_{\mathbb{R}} e^{\frac{i}{h} w \xi} w_{0}\left(x-\frac{w}{2}\right) w_{0}\left(x+\frac{w}{2}\right)^{*} d w \frac{d x d \xi}{4 \pi^{2}}= \\
& \frac{1}{2 \pi} \int_{\mathbb{R}} w_{0}(x) w_{0}(x)^{*} d x=\frac{h}{2 \pi} I_{\mathbb{C}^{2}}
\end{aligned}
$$

Inserting this in (6.7) gives

$$
J_{z_{1}}^{0}(z, h)=\frac{h}{\pi}\left(z-\kappa\left(n_{1} h, h\right)\right)^{-1} .
$$

To analyze the remaining contribution to (6.2) we use (5.36) to write

$$
\begin{gathered}
\left(E_{-+}(z, h)^{-1}-\left(z-\kappa\left(n_{1} h, h\right)\right)^{-1} I_{\mathbb{C}^{2}}\right) v_{+}(\gamma)=\sum_{\alpha} e(\gamma-\alpha) v_{+}(\alpha), \\
e(\gamma)=e(z, h, \gamma)=\mathcal{O}\left(h^{\infty}\langle\gamma\rangle^{-\infty}\right), \quad|\operatorname{Im} z|>h^{M} .
\end{gathered}
$$

Hence $J_{z_{1}}(z, h)=J_{z_{1}}^{0}(z, h)+J_{z_{1}}^{1}(z, h)$ where,

$$
J_{z_{1}}^{1}(z, h)=\int_{\mathbb{T}_{*}^{2}} \operatorname{tr}_{\mathbb{C}^{2}} \sigma\left(E_{+}(z, h)\left(E_{-+}(z, h)^{-1}-\left(z-\kappa\left(n_{1} h, h\right)\right)^{-1} I_{\mathbb{C}^{2}}\right) E_{-}(z, h)\right) \frac{d x d \xi}{4 \pi^{2}} .
$$


Lemma 5.5 and (5.44) give

$$
\begin{gathered}
E_{+}(z, h) v_{+}(x)=\sum_{\gamma} r_{\gamma} W_{+}(x) v_{+}(\gamma), \quad W_{+}=w_{0}+e_{0}, \quad e_{0}=\mathcal{O}\left(h^{\infty}\right)_{\mathscr{S}} \\
\left(E_{-}(z, h) v\right)(\gamma)=\left\langle v, r_{\gamma} W_{-}\right\rangle, \quad W_{-}=w_{0}+f_{0}, \quad f_{0} \in \mathcal{O}\left(h^{\infty}\right)_{\mathscr{S}}
\end{gathered}
$$

so that, using (6.8) again,

$$
\begin{aligned}
J_{z_{1}}^{1}(z, h) & =\sum_{\gamma, \beta} \int_{\mathbb{T}_{*}^{2}} \int_{\mathbb{R}} \operatorname{tr}_{\mathbb{C}^{2}} E_{+}\left(x-\frac{w}{2}, \gamma\right) e(\gamma-\beta) E_{-}\left(\beta, x+\frac{w}{2}\right) e^{\frac{i}{h} w \xi} d w \frac{d x d \xi}{4 \pi^{2} h} \\
& =\sum_{\gamma, \beta} \int_{\mathbb{T}_{*}^{2}} \int_{\mathbb{R}} r_{\gamma+\beta} W_{+}\left(x-\frac{w}{2}\right) e(\gamma) r_{\beta} W_{-}\left(x+\frac{w}{2}\right)^{*} e^{\frac{i}{h} w \xi} d w \frac{d x d \xi}{4 \pi^{2}} .
\end{aligned}
$$

As in (6.9) we now use the sum over $\beta_{2}$ to change integration in $\xi$ from $\mathbb{T}_{*}^{1}$ to $\mathbb{R}$ and then integrate in $w$ and $\xi$. This and (6.10) give

$$
\begin{aligned}
J_{z_{1}}^{1}(z, h) & =\frac{h}{2 \pi} \sum_{\gamma} \sum_{\beta_{1}} \int_{\mathbb{T}_{*}^{1}} e^{i x \gamma_{2}} W_{+}\left(x-\beta_{1}-\gamma_{1}\right) e(\gamma) W_{-}\left(x-\beta_{1}\right) d x \\
& =\mathcal{O}\left(h^{\infty}\right) \sum_{\gamma} \int_{\mathbb{R}}\left|W_{+}\left(x-\gamma_{1}\right)\right|\langle\gamma\rangle^{-\infty}\left|W_{-}(x)\right| d x=\mathcal{O}\left(h^{\infty}\left\|W_{-}\right\|\left\|W_{+}\right\|\right) .
\end{aligned}
$$

The following proposition summarizes what we have done in this section so far:

Proposition 6.1. Suppose that $Q$ is given by (4.30) and that $\widehat{\mathrm{tr}}$ is defined in (4.3). Let $z_{1}$ be chosen as in (5.29). Then

$$
\begin{gathered}
\widehat{\operatorname{tr}}\left(Q^{\mathrm{w}}(x, h D)-z\right)^{-1}:=\frac{h}{\pi} \sum_{n \in \mathbb{Z}}(z-\kappa(h n ; h))^{-1}+F_{z_{1}}(z, h)+\mathcal{O}\left(h^{\infty}\right), \\
|\operatorname{Im} z|>h^{M}, \quad\left|z-z_{0}\right| \leq \varepsilon_{0} h
\end{gathered}
$$

where $F_{z_{1}}(z, h)$ is holomorphic in $\left|z-z_{0}\right| \leq \varepsilon_{0} h, M$ is arbitrary and $\kappa(n h, n)$ defined by (5.7).

Remark 7. Using one variable complex analysis, a crude estimate $G_{z_{1}}(z, h)=\mathcal{O}\left(h^{-M_{0}}\right)$ and maximum principle similar to [DyZw2, Lemma D.1] one can show that (6.11) holds in a fixed neighbourhood of the Dirac point $\left.\Delta\right|_{B_{k}} ^{-1}(0)$ with $F$ independent of $z_{1}$ and holomorphic. As in [HS90b] we opt for a simpler version of piecing together local expressions (6.11) using a partition of unity.

We are now in the position to prove the main theorem describing the semiclassical density of states formula for our model of graphene: 
Theorem 1. Let $z^{D}:=\left.\Delta\right|_{B_{k}} ^{-1}(0)$ be the energy of the Dirac points located on the $k$-th band. If $I$ is a sufficiently small neighbourhood of $z^{D}$, then for $f \in C_{\mathrm{c}}^{\alpha}(I)$

$$
\tilde{\operatorname{tr}} f\left(H_{B}\right)=\frac{h}{\pi\left|b_{1} \wedge b_{2}\right|} \sum_{n \in \mathbb{Z}} f\left(z_{n}(h)\right)+\mathcal{O}\left(\|f\|_{C^{\alpha}} h^{\infty}\right), \quad \Delta\left(z_{n}(h)\right)=\kappa(n h, h),
$$

where $\kappa(n h, h)$ is given by $(5.7)$.

Proof. We cover $I$ by intervals of type $I_{z_{1}}^{1}:=\left(\left.\Delta\right|_{B_{k}}\right)^{-1}\left(\left(z_{1}-\varepsilon_{0} h, z_{1}+\varepsilon_{0} h\right)\right)$ where $z_{1}$ is as in (5.29), and intervals $I_{z_{2}}^{2}:=\left(\left.\Delta\right|_{B_{k}}\right)^{-1}\left(\left(z_{2}-\varepsilon_{1} h, z_{2}+\varepsilon_{1} h\right)\right)$ where $\left(z_{2}-2 \varepsilon_{1} h, z_{2}+\right.$ $\left.2 \varepsilon_{1} h\right) \cap \operatorname{Spec}\left(Q_{0}(x, h D)\right)=\emptyset\left(Q_{0}\right.$ is defined in (5.2)). Lemma 5.2 shows that near intervals $\Delta\left(I_{z_{2}}^{2}\right), \widehat{\operatorname{tr}}\left(Q^{\mathrm{w}}(x, h D)-z\right)^{-1}$ is holomorphic. Since we are also away from $\kappa(h n ; h)^{\prime} s$, that means that $(6.11)$ holds also near $I_{z_{2}}^{2}$.

Following [HS90b, §10] we proceed in two steps. First we recall that for $f \in C_{\mathrm{c}}^{\infty}(\mathbb{R})$ satisfying

$$
f^{(k)}=\mathcal{O}\left(h^{-N_{0}}\right) \text { for a fixed } N_{0} \text { and } 0 \leq k \leq 4
$$

we can find an extension of $f, \tilde{f} \in C_{\mathrm{c}}^{\infty}(\mathbb{C})$ satisfying

$$
\widetilde{f}, \tilde{f}^{\prime}=\mathcal{O}\left(h^{-N_{0}}\right), \quad \partial_{\bar{z}} \tilde{f}=\mathcal{O}\left(h^{-N_{0}}|\operatorname{Im} z|\right),
$$

In fact, Mather's construction of $\widetilde{f}-$ see $(4.13)$ - shows that

$$
\partial_{\bar{z}} \tilde{f}=|\operatorname{Im} z| \mathcal{O}\left(\left\|\xi^{2} \hat{f}(\xi)\right\|_{L^{1}(d \xi)}\right)=|\operatorname{Im} z| \mathcal{O}\left(|\operatorname{supp} f| \sup _{k \leq 4}\left|f^{(k)}\right|\right)
$$

and (6.13) implies (6.14).

Using a partition of unity with functions supported in intervals of type $I_{z_{j}}^{j}, j=1,2$, covering $I$, we only need to consider $f$ supported in $I_{z_{j}}^{j}$ and satisfying (6.13).

If $I$ is a sufficiently small neighbourhood of the Dirac point $z^{D}=\left.\Delta\right|_{B_{k}} ^{-1}(0)$, we obtain no Dirichlet contribution in (4.33). (The Dirichlet spectrum is located at the band edges $\Delta(z)= \pm 1$.) We observe further that $\Delta$ has a non-vanishing derivative inside the $k$-th band and $1 /|\operatorname{Im} \Delta(z)| \sim 1 /|\operatorname{Im} z|$. Inserting (6.11) into (4.33) and using a generalized version of the argument principle, as in the proof of Lemma 4.10, we obtain

$$
\begin{aligned}
\tilde{\operatorname{tr}}\left(f\left(H^{B}\right)\right)= & \frac{h}{\left|b_{1} \wedge b_{2}\right| \pi^{2}} \int_{\mathbb{C}} \partial_{\bar{z}} \tilde{f}(z) \Delta^{\prime}(z) \sum_{n \in \mathbb{Z}}(z-\kappa(h n, n))^{-1} d m(z) \\
& \quad+\frac{1}{\pi} \int_{|\operatorname{Im} z|<h^{M}} \partial_{\bar{z}} \widetilde{f}(z) \mathcal{O}(1 /|\operatorname{Im} z|) d m(z) \\
= & \frac{h}{\pi\left|b_{1} \wedge b_{2}\right|} \sum_{n \in \mathbb{Z}} f\left(z_{n}(h)\right)+\mathcal{O}\left(h^{M-N_{0}}\right), \quad z_{n}(h)=\left.\Delta\right|_{B_{k}} ^{-1}(\kappa(n h, h)) .
\end{aligned}
$$


We now approximate $f \in C_{\mathrm{c}}^{\alpha}(I)$ by

$$
f_{h}(x)=h^{-M_{0}} \int_{\mathbb{R}} f(y) \psi\left(h^{-M_{0}}(x-y)\right) d y, \quad \psi \in C_{\mathrm{c}}^{\infty}(\mathbb{R} ;[0,1]), \quad \int \psi(y) d y=1 .
$$

The condition (6.13) is then satisfied with $N_{0}=4 M_{0}$. Since $f \in C^{\alpha}$ we also have

$$
\sup _{x}\left|f(x)-f_{h}(x)\right| \leq\|f\|_{C^{\alpha}} h^{\alpha M_{0}} .
$$

By using (6.15) with $f$ replaced by $f_{h}$ and then using (6.16)

$$
\tilde{\operatorname{tr}} f\left(H_{B}\right)=\frac{h}{\pi\left|b_{1} \wedge b_{2}\right|} \sum_{n \in \mathbb{Z}} f\left(z_{n}(h)\right)+\mathcal{O}\left(\|f\|_{C^{\alpha}} h^{\alpha M_{0}-1}\right)+\mathcal{O}\left(\|f\|_{C^{\alpha}} h^{M-4 M_{0}}\right) .
$$

By choosing $M=5 M_{0}$ and then $M_{0}$ arbitrarily large we obtain (6.12).

Things become much simpler when $f$ is smooth. For completeness we include

Theorem 2. Suppose that $f \in C_{\mathrm{c}}^{\infty}(I)$ where $I$ is a small neighbourhood of a Dirac energy $z^{D}$. Then for any $N$

$$
\widetilde{\operatorname{tr}} f\left(H_{B}\right)=\sum_{j=1}^{N} A_{j}(f) h^{j}+\mathcal{O}\left(h^{N+1}\right), \quad A_{0}(f)=\rho_{0}(f), \quad A_{1}(f)=0 .
$$

Proof. We use the method of [DS99, Chapter 7] and consider an almost analytic extension of $f$ defined by (4.13). Then, avoiding again the Dirichlet eigenvalues by taking $I$ small enough,

$\tilde{\operatorname{tr}} f\left(H_{B}\right)=\frac{2}{(2 \pi)^{2} 3 \sqrt{3} \pi} \int_{\mathbb{R}^{2} / 2 \pi \mathbb{Z}^{2}}\left(\int_{\mathbb{C}} \partial_{\bar{\lambda}} \widetilde{f}(\lambda) \Delta^{\prime}(\lambda) \operatorname{tr}_{\mathbb{C}^{2}} \sigma\left(\left(Q^{\mathrm{w}}-\Delta(\lambda)\right)^{-1}\right) d m(\lambda)\right) d x d \xi$,

which follows from Definition 4.3 and (4.33). From [DS99, Proposition 8.6] we have, for $z \in D(0, C) \backslash \mathbb{R}$ (and any fixed $C$ ),

$$
\begin{gathered}
\left(Q^{\mathrm{w}}-z\right)^{-1}=R^{\mathrm{w}}\left(z ; x, h D_{x}, h\right) \\
\left|\partial_{x}^{\alpha} \partial_{\xi}^{\beta} R(z, x, \xi, h)\right| \leq C_{\alpha \beta} \max (1, h /|\operatorname{Im} z|)^{3}|\operatorname{Im} z|^{-1-|\alpha|-|\beta|} .
\end{gathered}
$$

Hence in the formula for $\widetilde{\operatorname{tr}} f\left(H_{B}\right)$ we can replace $\sigma\left(Q^{\mathrm{w}}-\Delta(\lambda)\right)^{-1}$ by $R(\Delta(\lambda), x, \xi, h)$. As in $[\mathrm{DS99},(8.14)]$ we see that for $|\operatorname{Im} \Delta(\lambda)| \simeq|\operatorname{Im} \lambda| \geq h^{\delta}, 0<\delta<\frac{1}{2}$, we have an expansion

$$
\begin{gathered}
\operatorname{tr}_{\mathbb{C}^{2}} R(\Delta(\lambda), x, \xi, h) \sim \operatorname{tr}_{\mathbb{C}^{2}}(Q(x, \xi)-\Delta(\lambda))^{-1}+h^{2} \operatorname{tr}_{\mathbb{C}^{2}} q_{2}(\Delta(\lambda), x, \xi)(Q(x, \xi)-\Delta(\lambda))^{-5} \\
+h^{3} \operatorname{tr}_{\mathbb{C}^{2}} q_{3}(\Delta(\lambda), x, \xi)(Q(x, \xi)-\Delta(\lambda))^{-7}+\cdots,
\end{gathered}
$$

where $q_{j}(z, x, \xi) \in \mathbb{C}^{2} \otimes \mathbb{C}^{2}$ are polynomials in $z$ of degree $\leq 2 j$ and the coefficients are $(2 \pi \mathbb{Z})^{2}$ periodic. 
Adapting the calculation in [DS99, (8.16)] gives the expansion (6.17) with

$$
A_{j}(f)=\left.\sum_{ \pm} \frac{2}{(2 \pi)^{2} 3 \sqrt{3}} \frac{1}{(2 j) !} \int_{\mathbb{R}^{2} / 2 \pi \mathbb{Z}^{2}} \operatorname{tr}_{\mathbb{C}^{2}} \partial_{z}^{2 j}\left(q_{j}(z, x, \xi) f(z)\right)\right|_{z=z_{ \pm}(x, \xi)} d x d \xi
$$

where

$$
z_{ \pm}(x, \xi):=\Delta^{-1}\left( \pm \frac{1}{3}\left|1+e^{i x}+e^{i \xi}\right|\right)
$$

In particular,

$$
A_{0}(f)=\sum_{ \pm} \frac{2}{(2 \pi)^{2} 3 \sqrt{3}} \frac{1}{(2 j) !} \int_{\mathbb{R}^{2} / 2 \pi \mathbb{Z}^{2}} f\left(z_{ \pm}(x, \xi)\right) d x d \xi
$$

which is $\rho_{0}(f)$ for $f$ supported near $z_{D}$.

\section{Magnetic oscillations}

In this section we show how Theorem 1 can be used to describe low temperature magnetic oscillations in the (smoothed-out) density of states and in magnetization. In the physics literature they are known as the Shubnikov-de Haas $(\mathrm{SdH})$ and the de Haas-van Alphen (dHvA) effects, respectively.

We stress the asymmetry with respect to the Dirac energy levels which comes from semiclassical quantization conditions and the dispersion relations. It is not seen when a "perfect cone" (that is, a harmonic oscillator) approximation is used - see (1.2). We note that an asymmetry is already present in the case when there is no magnetic field. An experimental result in the setting molecular graphene $\left[G^{*} 12\right.$, Figure $\left.4 d\right]$ is shown in Figure 7. The corrections to the perfect cone approximation are due to the modified linear dispersion relation as energies move away from the Dirac points. The perfectly linear dispersion relation of the $\mathrm{QED}_{2+1}$-model has been a ubiquitous assumption in the physics literature - see Gusynin-Sharapov [SGB04], [GS05], [GS06] and references therein. The approach presented here leads to modified Landau levels showing the well known $\sqrt{n B}$-scaling only to leading order.

7.1. Shubnikov-de Haas oscillations in DOS. The Shubnikov-de Haas (SdH) effect is the occurrence of oscillations in the density of states, with periods proportional to the inverse strength of the magnetic field. These oscillations can be experimentally measured in terms of longitudinal conductivity or resistivity [W11] and [Tan11]. For a theoretical discussion of the relation between oscillations in electric and also thermal conductivities on the one hand and the density of states on the other hand, see also [GS05].

We start with an approximation for the semiclassical Landau levels $z_{h}$ of $H^{B}$ introduced in Theorem 6.1. For that we consider an approxiate Bohr-Sommerfeld condition:

$$
g\left(z_{n}^{(1)}(h)\right)=|n| h, \quad g(x):=\left.F_{0}\left(\Delta(x)^{2}\right)\right|_{I_{\delta, k}},
$$




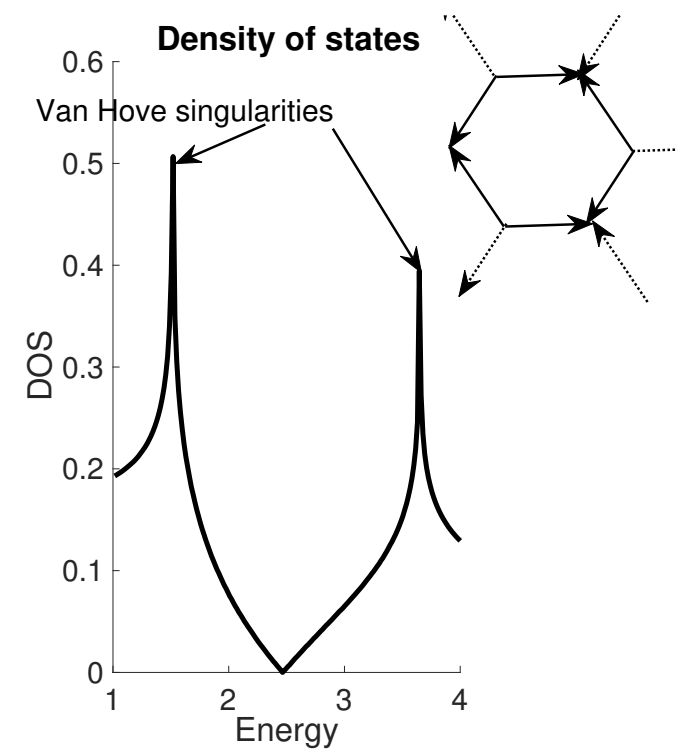

(A) The DOS of the operator $H^{B=0}$ (3.4) per hexagonal cell volume with zero magnetic field potential $\left(V_{e}\right)=0$ on the first Hill band $\left[0, \pi^{2}\right]$ as described in (2.17).

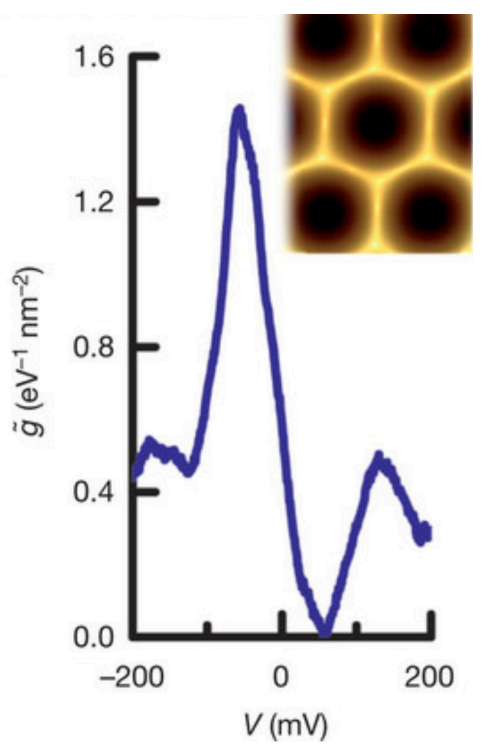

(B) An experimental plot of the density of states for a molecular model of graphene obtained using scanning tunneling microscopy [G*12].

FiguRE 7. Comparing numerical and experimental no-magnetic field DOS in the quantum graph model and molecular graphene, respectively.

where $F_{0}$ is the normalized phase space area of one potential well in the Brouillon zone defined in Proposition 5.1 and $I_{\delta, k}$ as in Theorem 1. Since $F_{0}^{\prime}(0) \neq 0, \Delta\left(z_{D}\right)=0$, $\Delta^{\prime}\left(z_{D}\right) \neq 0$ (see $(2.17)$ ), we have $g\left(z_{D}\right)=g^{\prime}\left(z_{D}\right)=0, g^{\prime \prime}\left(z_{D}\right)>0$. This means that we have two branches of the inverse of $g$ defined for small $x \geq 0$ : $\pm\left(g_{ \pm}^{-1}(x)-z_{D}\right) \geq 0$. Then

$$
z_{ \pm|n|}^{(1)}(h)=g_{ \pm}^{-1}(|n| h), \quad z_{0}^{(1)}(h)=0 .
$$

Remark 8. Because of the asymmetry of the cones which are the solutions to $\mid Q(x, \xi)-$ $\Delta(z) \mid=0$ in a neighbourhood of the Dirac point $\left.\Delta\right|_{B_{k}} ^{-1}(0)$, we observe that although $\kappa(n h, h)=-\kappa(-n h, h)$ we have $z_{n}^{(1)}(h) \neq-z_{-n}^{(1)}(h)+\mathcal{O}\left(h^{\infty}\right)$ in general. That can already be seen in the simplest case (2.16).

We recall from (5.6) that

$$
F\left(\Delta\left(z_{n}(h)\right)^{2}\right)=F_{0}\left(\Delta\left(z_{n}(h)\right)^{2}\right)+\mathcal{O}\left(h^{2} \Delta\left(z_{n}(h)\right)^{2}\right)=|n| h+\mathcal{O}\left(h^{\infty}\right),
$$

which gives $\Delta\left(z_{n}(h)\right)^{2}=\Delta\left(z_{n}^{(1)}(h)\right)^{2}+\mathcal{O}\left(|n| h^{3}\right)+\mathcal{O}\left(h^{\infty}\right)$. Hence,

$$
z_{n}(h)=z_{n}^{(1)}(h)+\mathcal{O}\left(h^{\frac{5}{2}}|n|^{\frac{1}{2}}\right), \quad n \neq 0
$$




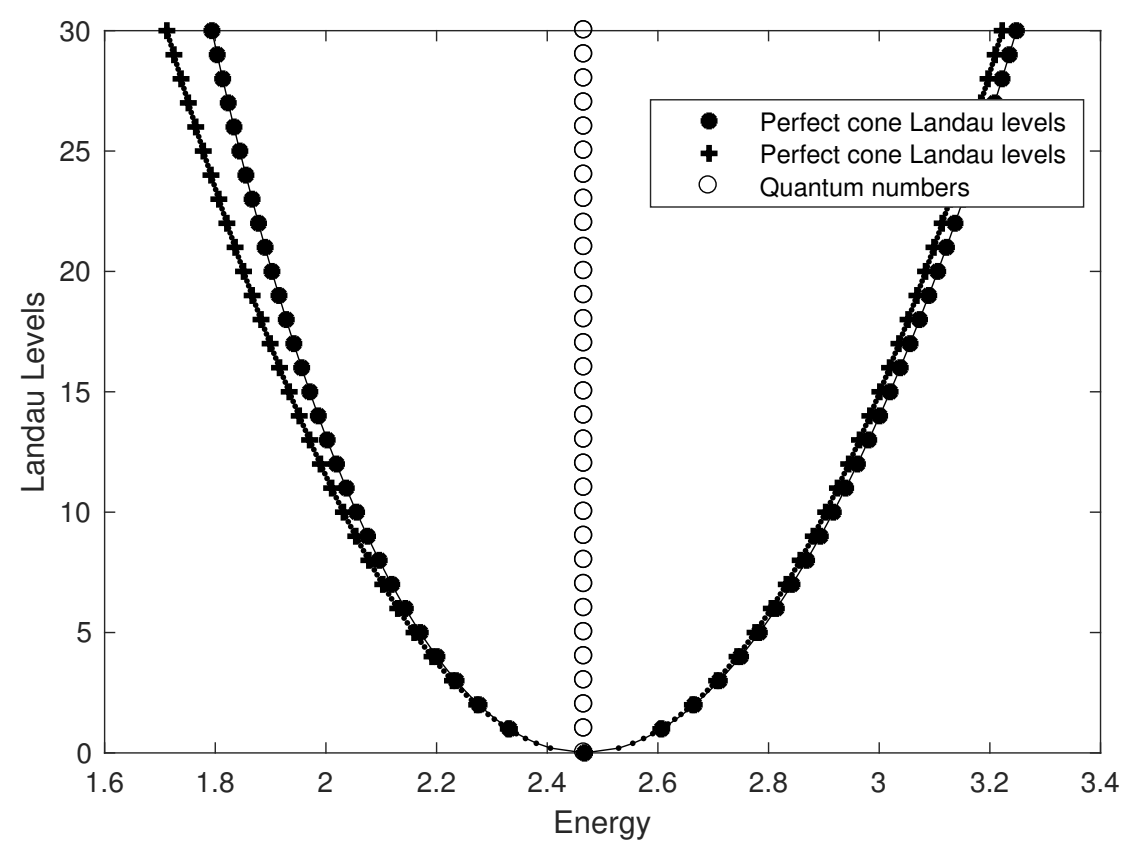

FiguRE 8. Landau levels located on the Dirac cone of the first Hill band with zero potential derived from the Bohr-Sommerfeld condition and normalized phase space area $g(7.1)$ and its perfect cone approximation (7.5) for magnetic flux $h=0.01$.

For $f \in C^{\alpha}(I), 0<\alpha \leq 1$, we then have

$$
\rho_{B}(f)=\widetilde{\rho}_{B}(f)+\mathcal{O}\left(\|f\|_{C^{\alpha}} h^{2 \alpha}\right), \quad \widetilde{\rho}_{B}(f):=\frac{h}{\pi\left|b_{1} \wedge b_{2}\right|} \sum_{n \in \mathbb{Z}} f\left(z_{n}^{(1)}(h)\right) .
$$

The error term came from the approximation (7.3) and the fact that the number of terms contributing on the support of $f$ is bounded by $\mathcal{O}(1 / h)$ :

$$
h \sum_{n \neq 0}\left|f\left(z_{n}(h)\right)-f\left(z_{n}^{(1)}(h)\right)\right| \leq\|f\|_{C^{\alpha}} h^{1+\frac{5}{2} \alpha} \sum_{0<n \leq C / h} n^{\frac{1}{2} \alpha}=\mathcal{O}\left(\|f\|_{C^{\alpha}} h^{2 \alpha}\right) .
$$

The leading term in (7.4) provides a refinement of (1.2) which is easy to investigate numerically. To compare it with (1.2) we calculate $v_{F}$ (the value used here differs by the area factor) by using the leading term in the Taylor expansion of $g$ (and (5.1) to calculate $\left.F_{0}^{\prime}(0)\right)$ :

$$
g(x)=\Delta^{\prime}\left(z_{D}\right)^{2} F_{0}^{\prime}(0) x^{2}+\mathcal{O}\left(x^{3}\right), \quad F_{0}^{\prime}(0)=3^{\frac{3}{2}} \Longrightarrow v_{F}=3^{-\frac{3}{4}} \Delta^{\prime}\left(z_{D}\right)^{-1} .
$$



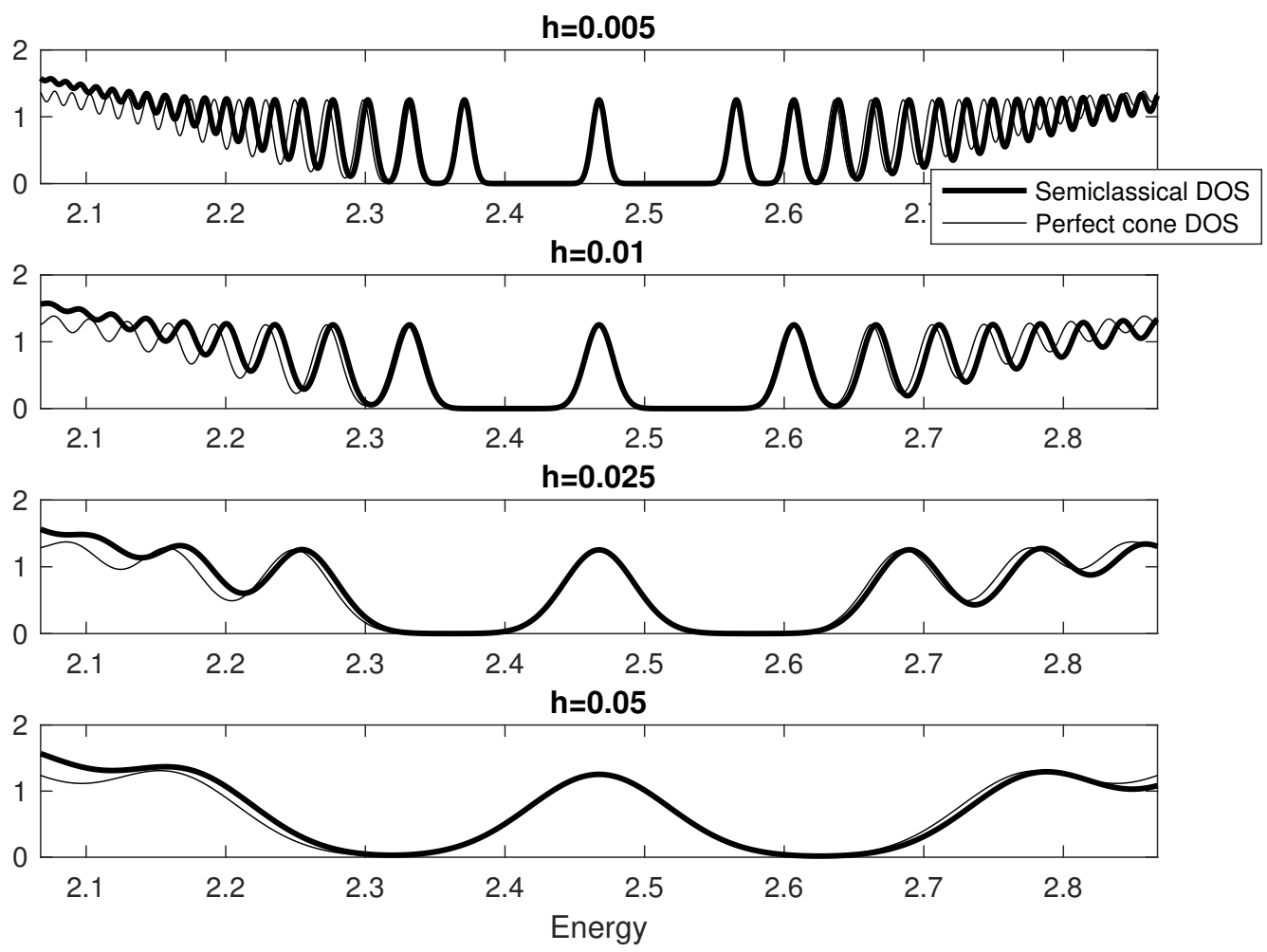

Figure 9. The plots of $\mu \mapsto \widetilde{\rho}_{B}\left(\exp \left((\bullet-\mu)^{2} / 2 \sigma^{2}\right) / \sqrt{2 \pi} \sigma\right)$ for different values of $h$ and $\sigma=h$ (hence pushing the validity of (7.4); see also Figure 2). We note the asymmetry when compared to the density of states obtained using the perfect cone approximation (7.5).

In other words, a "perfect cone" quantization condition reads,

$$
\begin{gathered}
g_{\mathrm{c}}\left(z_{n}^{\mathrm{c}}(h)\right)=|n| h, \quad g_{\mathrm{c}}(x)=v_{F}^{-2}\left(x-z_{D}\right)^{2}, \quad v_{F}=3^{-\frac{3}{4}} \Delta^{\prime}\left(z_{D}\right)^{-1}, \\
z_{n}^{\mathrm{c}}=z_{D}+v_{F} \operatorname{sgn}(n) \sqrt{|n| h},
\end{gathered}
$$

and the comparison with (7.1) is shown in Figure 8.

To plot the density of states we use $\widetilde{\rho}_{B}(f)$ in $(7.4)$ with $f_{\mu}(x)=e^{-(x-\mu)^{2} / 2 \sigma^{2}} / \sqrt{2 \pi} \sigma$ and plot $\mu \mapsto \widetilde{\rho}_{B}\left(f_{\mu}\right)$. Since $\left\|f_{\mu}\right\|_{C^{1}}=\mathcal{O}\left(\sigma^{-2}\right)$ we obtain valid approximation for $\sigma \gg h$ - see Figure 9

7.2. De Hass-van Alphen oscillations. As first discovered by de Haas and van Alphen in 1930, magnetization and magnetic susceptibility of three dimensional metals oscillate as functions of $1 / B$. They were not aware that Landau had just predicted presence of such oscillations. The frequencies are proportional to the areas of the extremal cross sections of the Fermi surface in the direction of the magnetic field. This 
explanation was provided by Onsager [052] and a rigorous mathematical proof was given by Helffer and Sjöstrand [HS90b].

In the case of graphene, the dHvA effect does not seem to be well understood neither experimentally nor theoretically [L11]. This is partly due to difficulties in accounting for all the parameters of the system: for instance, in the grand-canonical ensemble is frequently used to model the dHvA effect [SGB04], the chemical potentials are assumed to be independent of the external magnetic field. For a thorough discussion of this assumption, also made in this paper, we refer to [CM01]. (We comment that the assumption of having a constant chemical potential is also assumed in the 3D LifshitzKosevich theory [KF17] for the study of magnetic oscillations in the susceptibility of metals at low temperatures. A 2D analogue of the theory for metals has been developed by Shoenberg [S84] and was discussed in the context of graphene in [L11].)

Compared to previous discussions of magnetic oscillations - see for instance [SGB04] and [L11] - where the limit of infinitely many "perfect cone" Landau levels was considered, we are only going to assume that there are finitely many semiclassically corrected Landau levels.

To introduce magnetization, we first define the grand-canonical potential at temperature $T=1 / \beta$. Since we are interested in chemical potentials (energy) near the Dirac energy, we choose a smooth function $\eta \in C_{\mathrm{c}}^{\infty}(I)$ which is equal to 1 in a neighbourhood of the Dirac energy and replace $\rho_{B}$ by $\eta \rho_{B}$ we then define

$$
\Omega_{\beta}(\mu, h):=\rho_{B}\left(\eta(\bullet) f_{\beta}(\mu-\bullet)\right), \quad f_{\beta}(x):=-\beta^{-1} \log \left(e^{\beta x}+1\right) .
$$

We note that $f_{\infty}(x)=-x_{+}$and we define $\Omega_{\infty}$ using that function. Since $f_{\infty}$ is a Lipschitz function, Theorem 1 implies that

$$
\Omega_{\beta}(\mu, h)=\frac{h}{\pi\left|b_{1} \wedge b_{2}\right|} \sum_{n \in \mathbb{Z}} f_{\beta}\left(\mu-z_{n}(h)\right) \eta\left(z_{n}(h)\right)+\mathcal{O}\left(h^{\infty}\right),
$$

which holds true for $\Omega_{\infty}$ defined using $f_{\infty}=-x_{+}$. The function $x \mapsto f_{\beta}(\mu-x)$ is uniformly smooth away from $x=\mu$. For $\mu$ 's near $z_{D}$, changing $\eta$ gives uniformly smooth contributions (in $\mu$ and $h$ ) - see Theorem 2.

Remark 9. The grand-canonical potential at non-zero temperatures (finite values of $\beta$ ) can be recovered from $\Omega_{\infty}$ using the Fermi distribution $n_{\beta}$ :

$$
\Omega_{\beta}(\mu, h)=\left(-n_{\beta}^{\prime} * \Omega_{\infty}(\bullet, h)\right)(\mu), \quad n_{\beta}(x):=\left(1+e^{\beta x}\right)^{-1} .
$$

Indeed, we easily check that $\left(-(\bullet-x)_{+} * n_{\beta}^{\prime}\right)(\mu)=f_{\beta}(x)$.

Magnetization is defined as

$$
M_{\beta}(\mu, h):=-\left|b_{1} \wedge b_{2}\right| \frac{\partial}{\partial h} \Omega_{\beta}(\mu, h) .
$$


If we consider the full expansion of the levels $z_{n}(h)$ (obtained from $F(\omega, h)$ in Proposition 5.1) we could analyse $M_{\beta}$ for $\beta<h^{-M_{0}}$ for any fixed $M_{0}$ - see the remarks after [HS90b, Theorem 10.2].

To avoid technical complications, we will instead, similarly to [HS90b], consider formal magnetization obtained using leading term DOS, $\widetilde{\rho}_{B}$, from (7.4). That already shows the sawtooth pattern derived in [SGB04] using the "perfect cone" approximation - see Theorem 3 and Figure 10. Remarkably it also agrees with the "exact" spectral numerical calculation explained in $\S 7.3$ - see Figure 13.

Let us now consider chemical potentials located on the upper cone of the first Hill band, i.e. $\mu \in\left[z_{D},\left.\Delta\right|_{B_{1}} ^{-1}\left(-\frac{1}{3}\right)\right]$. Formal grand-canonical potential and formal magnetization are obtained from (7.7) and (7.9) by replacing $\left(z_{n}(h)\right)$ with the semiclassical Landau levels $\left(z_{n}^{(1)}\right)$ given by the leading order Bohr-Sommerfeld condition (7.1), and thus defined as follows

$$
\begin{gathered}
\omega_{\beta}(\mu, h):=\frac{h}{\pi\left|b_{1} \wedge b_{2}\right|} \sum_{n \in \mathbb{Z}} f_{\beta}\left(\mu-z_{n}^{(1)}(h)\right) \eta\left(z_{n}^{1}(h)\right), \\
m_{\beta}(\mu, h):=-\left|b_{1} \wedge b_{2}\right| \frac{\partial}{\partial h} \omega_{\beta}(\mu, h),
\end{gathered}
$$

and

$$
\eta(x)=\Theta_{\frac{1}{2}}(x):= \begin{cases}0 & x<z_{D} \\ \frac{1}{2} & x=z_{D} \\ 1 & z_{D}<x<\left.\Delta\right|_{B_{1}} ^{-1}\left(-\frac{1}{3}\right) \\ 0 & x \geq\left.\Delta\right|_{B_{1}} ^{-1}\left(-\frac{1}{3}\right) .\end{cases}
$$

(This non-smooth $\eta$ is convenient for spectral calculations and hence comparing semiclassical and exact numerics. The energy $\left.\Delta\right|_{B_{1}} ^{-1}\left(-\frac{1}{3}\right)$ corresponds to the energetic upper end of the upper cone.)

The construction for chemical potentials on the lower cone of the first Hill band, i.e. $\mu \in\left[\left.\Delta\right|_{B_{1}} ^{-1}\left(\frac{1}{3}\right), z_{D}\right]$, is similar. Using the cut-off function

$$
\eta=\mathbb{1}_{\left[\left.\right|_{B_{1}} ^{-1}\left(\frac{1}{3}\right), z_{D}\right]}\left(1-\Theta_{\frac{1}{2}}\right),
$$

we obtain the semiclassical approximation from Landau levels located on the lower cone at zero temperature

$$
\omega_{\infty}(\mu, h):=\frac{h}{\pi\left|b_{1} \wedge b_{2}\right|} \sum_{n \in \mathbb{Z}}\left(\mu-g^{-1}(n h)\right)_{-} \eta\left(g^{-1}(n h)\right) .
$$

We compare the oscillations on the upper (7.10) and lower cone (7.12) at zero temperature showing the asymmetry between the two different cones in Figure 13. 

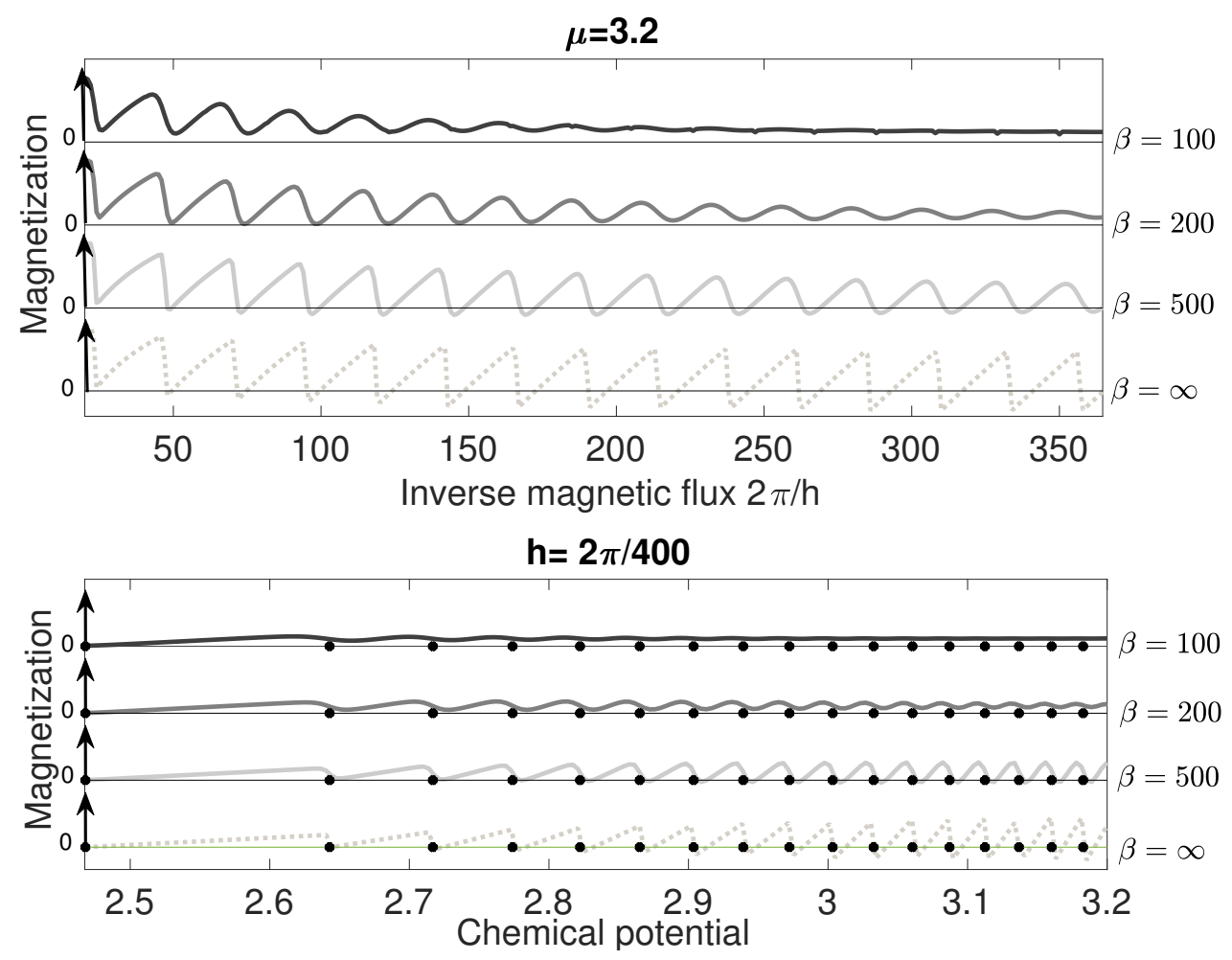

Figure 10. The magnetization (7.10) for different temperatures for a Hamiltonian with zero potential on the first Hill band. The sawtooth profile is clearly visible in the zero temperature limit $\beta=\infty$ and the oscillation period is approximately proportional to the inverse Fermi surface. As temperature increases, the oscillations become more smooth as predicted in (7.8) and the oscillation amplitude decreases. For zero temperature we see that the oscillation period increases linearly in $\mu$. This is no longer true when non-zero temperatures are considered.

The following asymptotic result shows the presence of "sawtooth" oscillations in magnetization.

Theorem 3. The formal magnetization for chemical potentials on the upper cone at zero temperature (defined in (7.10)) satisfies

$$
m_{\infty}(\mu, h)=\frac{1}{\pi} \sigma\left(\frac{g(\mu)}{h}\right) \frac{g(\mu)}{g^{\prime}(\mu)}+\mathcal{O}\left(h^{\frac{1}{2}}\right),
$$

where $g(x)=F_{0}\left(\Delta(x)^{2}\right)$, with $F_{0}$ given in (5.6), is the leading term in the BohrSommerfeld condition (7.1) and $\sigma$ is the sawtooth function,

$$
\sigma(y):=y-[y]-\frac{1}{2}
$$


Proof. Since in $(7.10) z_{n}^{(1)}(h)=g_{+}^{-1}(n h)$ (we drop + in what follows) and $\eta=\Theta_{\frac{1}{2}}$,

$$
\omega_{\infty}(\mu, h)=-\frac{h}{\pi\left|b_{1} \wedge b_{2}\right|}\left(\frac{1}{2}\left(\mu-z_{D}\right)+\sum_{n \geq 1}\left(\mu-g^{-1}(n h)\right)_{+}\right) .
$$

We rewrite the sum appearing in (7.15) as follows:

$$
\sum_{n \geq 1}\left(\mu-g^{-1}(n h)\right)_{+}=-\frac{1}{2} h\left(\mu-z_{D}\right)+\int_{z_{D}}^{\mu}\left(\frac{g(x)}{h}-\sigma\left(\frac{g(x)}{h}\right)\right),
$$

where $\sigma$ is defined by (7.14). In fact, both sides are 0 at $\mu=\left.\Delta\right|_{B_{k}} ^{-1}(0)$ and the derivative of the left hand side is

$$
\sum_{n \geq 0}\left(\mu-g^{-1}(n h)\right)_{+}^{0}=\left[\frac{g(\mu)}{h}\right]=\frac{g(\mu)}{h}-\sigma\left(\frac{g(x)}{h}\right)-\frac{1}{2} .
$$

This gives the following expression for $\omega$ :

$$
\begin{aligned}
\omega_{\infty}(\mu, h) & =-\frac{1}{\pi\left|b_{1} \wedge b_{2}\right|} \int_{\left.\Delta\right|_{B_{k}} ^{-1}(0)}^{\mu}\left(g(x)-h \sigma\left(\frac{g(x)}{h}\right)\right) \\
& =G(\mu)+\frac{h^{2}}{\pi\left|b_{1} \wedge b_{2}\right|} \int_{0}^{g(\mu) / h} \sigma(z)\left(g^{-1}\right)^{\prime}(z h) d z
\end{aligned}
$$

where $G(\mu)$ is independent of $h$. Hence,

$$
\begin{aligned}
m_{\infty}(\mu, h)-\frac{1}{\pi} \sigma\left(\frac{g(\mu)}{h}\right) \frac{g(\mu)}{g^{\prime}(\mu)} & =h \int_{0}^{g(\mu) / h} \sigma(z)\left(\left(g^{-1}\right)^{\prime \prime}(z h) z h+2\left(g^{-1}\right)^{\prime}(z h)\right) d z \\
& =h^{\frac{1}{2}} \int_{0}^{g(\mu) / h} \sigma(z) z^{-\frac{1}{2}} a(z h) d z,
\end{aligned}
$$

where $a(\xi):=\left(g^{-1}\right)^{\prime \prime}(\xi) \xi^{\frac{3}{2}}+2\left(g^{-1}\right)^{\prime}(\xi) \xi^{\frac{1}{2}}$. The function $a$ is smooth since $g(x)=$ $\left(G^{-1}\left(x-\left.\Delta\right|_{B_{k}} ^{-1}(0)\right)\right)^{2}$ where $G(0)=0, G^{\prime}(0) \neq 0$. That means that $g^{-1}(\xi)=\left.\Delta\right|_{B_{k}} ^{-1}(0)+$ $\xi^{\frac{1}{2}} \varphi(\xi), \varphi \in C^{\infty}$ so that $a(\xi)=\frac{3}{4} \varphi(\xi)+3 \xi \varphi^{\prime}(\xi)+\xi^{2} \varphi^{\prime \prime}(\xi) \in C^{\infty}$. We then write

$$
\int_{0}^{g(\mu) / h} \sigma(z) z^{-\frac{1}{2}} a(z h) d z=h^{\frac{1}{2}} \sum_{n=0}^{[g(\mu) / h]-1} \int_{n}^{n+1} \sigma(z) z^{-\frac{1}{2}} a(z h) d z+\mathcal{O}\left(h^{\frac{1}{2}}\right) .
$$

For $1 \leq n \leq c / h$

$$
\begin{aligned}
\int_{n}^{n+1} \sigma(z) z^{-\frac{1}{2}} a(z h) d z & =\int_{0}^{1} \sigma(z)(z+n)^{-\frac{1}{2}} a(h(z+n)) d z \\
& =n^{-\frac{1}{2}} \int_{0}^{1} \sigma(z)(1+z / n)^{-\frac{1}{2}} a(n h(1+z / n)) d z \\
& =n^{-\frac{1}{2}} a(n h) \int_{0}^{1} \sigma(z) d z+\mathcal{O}\left(n^{-\frac{3}{2}}\right)=\mathcal{O}\left(n^{-\frac{3}{2}}\right)
\end{aligned}
$$


Hence the sum on the right hand side of (7.18) is bounded and that concludes the proof of (7.13).

The leading term in (7.13) encapsulates the classical features of the dHvA effect: the function $\sigma(x)$ is periodic and its jump discontinuities coincide with the location of the Landau levels visible as the valleys in the lower Figure 10. The sawtooth profile shown in Figures 12 and 13 of the oscillations agrees with the results obtained in [SGB04] and [CM01] in which a sawtooth shape for magnetic oscillations in graphene was predicted. The quantity $g(\mu)$ is precisely the area enclosed by the Fermi curve as in the description of dHvA effect given by Onsager [O52]. In particular, this shows that the dHvA effect can be used as a test to study deviations from the perfect cone shape in graphene. Finally, the scaling factor $g(\mu) / g^{\prime}(\mu)$ implies a (at leading order) linear growth of the magnetic oscillations as a function of the chemical potential shown in Figure 10.

7.3. A Spectral approach to magnetic oscillations. It is well known that when the magnetic flux $h$ satisfies $h / 2 \pi \in \mathbb{Q}$, modified Floquet theory can be used to describe the spectrum of $H^{B}$ and the density of states. In particular, when $h=\frac{2 \pi p}{q}, p, q \in \mathbb{N}$, then the Floquet spectrum as a function of quasi-momentum $k$ can be calculated using $2 q \times 2 q$ matrices - see [BHJ17].

More precisely, for $k \in \mathbb{T}_{*}^{2}$ we follow [BHJ17] and define

$$
T_{q}(k):=\frac{1}{3}\left(\begin{array}{cc}
0 & \operatorname{id}_{\mathbb{C}^{q}}+e^{i k_{1}} J_{p, q}+e^{i k_{2}} K_{q} \\
\operatorname{id}_{\mathbb{C}^{q}}+e^{-i k_{1}} J_{p, q}^{*}+e^{-i k_{2}} K_{q}^{*} & 0
\end{array}\right)
$$

where

$$
\left(J_{p, q}\right)_{j \ell}=e^{\frac{2 \pi p}{q} i(\ell-1)} \delta_{j \ell}, \quad\left(K_{q}\right)_{j \ell}=\left\{\begin{array}{ccc}
1 & \ell \equiv j+1 \bmod q & 1 \leq j, \ell \leq q . \\
0 & \text { otherwise, }
\end{array}\right.
$$

Then $\lambda \in \operatorname{Spec}\left(H^{B}\right) \backslash \operatorname{Spec}\left(H^{D}\right)$ if and only if $\Delta(\lambda) \in \bigcup_{k \in \mathbb{T}_{*}^{2}} \operatorname{Spec}\left(T_{q}(k)\right)$. Thus, on each Hill band $H^{B}$ has $2 q$ non-overlapping bands that touch at the conical point. In particular, there are $q$ bands above and below the conical point.

The density of state is given in the following

Lemma 7.1. Let $h=2 \pi p / q$ then for any $f \in C_{c}\left(\mathbb{R} \backslash \operatorname{Spec}\left(H^{D}\right)\right)$

$$
\tilde{\operatorname{tr}}\left(f\left(H^{B}\right)\right)=\frac{1}{q\left|b_{1} \wedge b_{2}\right|} \int_{\mathbb{T}_{*}^{2} \Delta(\lambda) \in \operatorname{Spec}\left(T_{q}(k)\right)} f(\lambda) \frac{d k}{\left|\mathbb{T}_{*}^{2}\right|}
$$

Proof. Since the flux is of the form $h=\frac{2 \pi p}{q}$ there is a fundamental cell $W_{\Lambda}^{B}$ of measure $q\left|b_{1} \wedge b_{2}\right|$ with respect to which the operator $H^{B}$ is translational invariant [BHJ17]. Thus, along the lines of Lemma 4.4 we find that

$$
\widetilde{\operatorname{tr}}\left(f\left(H^{B}\right)\right)=\frac{1}{q\left|b_{1} \wedge b_{2}\right|} \operatorname{tr}\left(\mathbb{1}_{W_{\Lambda}^{B}} f\left(H^{B}\right)\right) .
$$


By Floquet theory, $f\left(H^{B}\right)$ is unitary equivalent to the bounded decomposable operator $\int_{\mathbb{T}_{*}^{2}}^{\oplus} f\left(H^{B}\right)(k) \frac{d k}{\left|\mathbb{T}_{*}^{2}\right|}$ such that for any orthonormal basis, $\left\{\varphi_{n}\right\}_{n \in \mathbb{N}}$, of $L^{2}\left(W_{\Lambda}^{B}\right)$

$$
\begin{aligned}
\tilde{\operatorname{tr}}\left(f\left(H^{B}\right)\right) & =\frac{1}{q\left|b_{1} \wedge b_{2}\right|} \operatorname{tr} \mathbb{1}_{W_{\Lambda}^{B}} f\left(H^{B}\right) \\
& =\frac{1}{q\left|b_{1} \wedge b_{2}\right|} \sum_{n \in \mathbb{N}}\left\langle\varphi_{n}, f\left(H^{B}\right)(k) \varphi_{n}\right\rangle_{L^{2}\left(\mathbb{T}_{*}^{2}, \frac{d k}{\left|\mathbb{T}_{*}^{2}\right|}\right) \otimes L^{2}\left(W_{\Lambda}^{B}\right)} \\
& =\frac{1}{q\left|b_{1} \wedge b_{2}\right|} \int_{\mathbb{T}_{*}^{2}} \operatorname{tr}_{L^{2}\left(W_{\Lambda}^{B}\right)} f\left(H^{B}\right)(k) \frac{d k}{\left|\mathbb{T}_{*}^{2}\right|} \\
& =\frac{1}{q\left|b_{1} \wedge b_{2}\right|} \int_{\mathbb{T}_{*}^{2}} \sum_{\lambda \in \operatorname{Spec}\left(H^{B}(k)\right)} f(\lambda) \frac{d k}{\left|\mathbb{T}_{*}^{2}\right|} .
\end{aligned}
$$

Away from $\operatorname{Spec}\left(H^{D}\right)$ the spectrum of $H^{B}(k)$ is characterized by $\Delta(\lambda) \in \operatorname{Spec}\left(T_{q}(k)\right)$ and (7.1) follows.

In the semiclassical regime $h \rightarrow 0$, the location of the energy bands of $\operatorname{Spec}\left(H^{B}\right)$ coincides with the location of the semiclassical Landau levels close to the conical point. By using the actual spectrum of $H^{B}$, the broadening of the Landau levels, known as Harper broadening [KH14], is already part of the model and does not have to be approximated as in [SGB04] or [CM01]. We should stress that Lemma 5.2 shows that the width of the bands is $O\left(h^{\infty}\right)$ and finer analysis of [HS88] could be used to show that the width is in fact $O\left(e^{-c / h}\right)$.

The advantage of the representation of the density of states in Lemma 7.1 is that we can calculate DOS numerically for larger values of $h$, that is, for strong magnetic fields. This approach is similar to the study of magnetic oscillations in the tight-binding model presented in [KH14].

Let the magnetic flux be of the form $h=2 \pi p / q$ with $p \in \mathbb{Z}, q \in \mathbb{N}$, then we study the grand-canonical potential localized to the spectrum on the first Hill band which by Lemma 7.1 satisfies

$$
\begin{aligned}
\Omega_{\beta}(\mu, h) & :=\left(f_{\beta} * \eta \rho_{B}\right)(\mu) \\
& =-\frac{1}{q\left|b_{1} \wedge b_{2}\right|} \frac{1}{\beta} \int_{\left.\mathbb{T}_{*}^{2} \Delta\right|_{B_{1}}(\lambda) \in \operatorname{Spec}\left(T_{q}(k)\right)} \log (\exp (\beta(\mu-\lambda))+1) \frac{d k}{\left|\mathbb{T}_{*}^{2}\right|} .
\end{aligned}
$$

Here $\eta$ is one on $\mathcal{A}:=\left.\Delta\right|_{B_{1}} ^{-1}\left(\bigcup_{k \in \mathbb{T}_{*}^{2}} \operatorname{Spec}\left(T_{q}(k)\right)\right.$ and zero on $\operatorname{Spec}\left(H^{B}\right) \backslash \mathcal{A}$. In the zero temperature limit this reduces to

$$
\Omega_{\infty}(\mu, h)=-\frac{1}{q\left|b_{1} \wedge b_{2}\right|} \int_{\left.\mathbb{T}_{*}^{2} \Delta\right|_{B_{1}}(\lambda) \in \operatorname{Spec}\left(T_{q}(k)\right)}(\mu-\lambda)_{+} \frac{d k}{\left|\mathbb{T}_{*}^{2}\right|} .
$$

Remarkably, $\Omega_{\infty}$ satisfies $\Omega_{\infty}(\mu, h)=\left(f_{\infty} * \rho_{B}\right)(\mu)$ without any cut-off for $\mu<\inf \operatorname{Spec}\left(H^{D}\right)$. The definition of the grand-canonical potential used here coincides with the expression in [GA03] up to the regularizing pre-factor $\left(q\left|b_{1} \wedge b_{2}\right|\right)^{-1}$. 


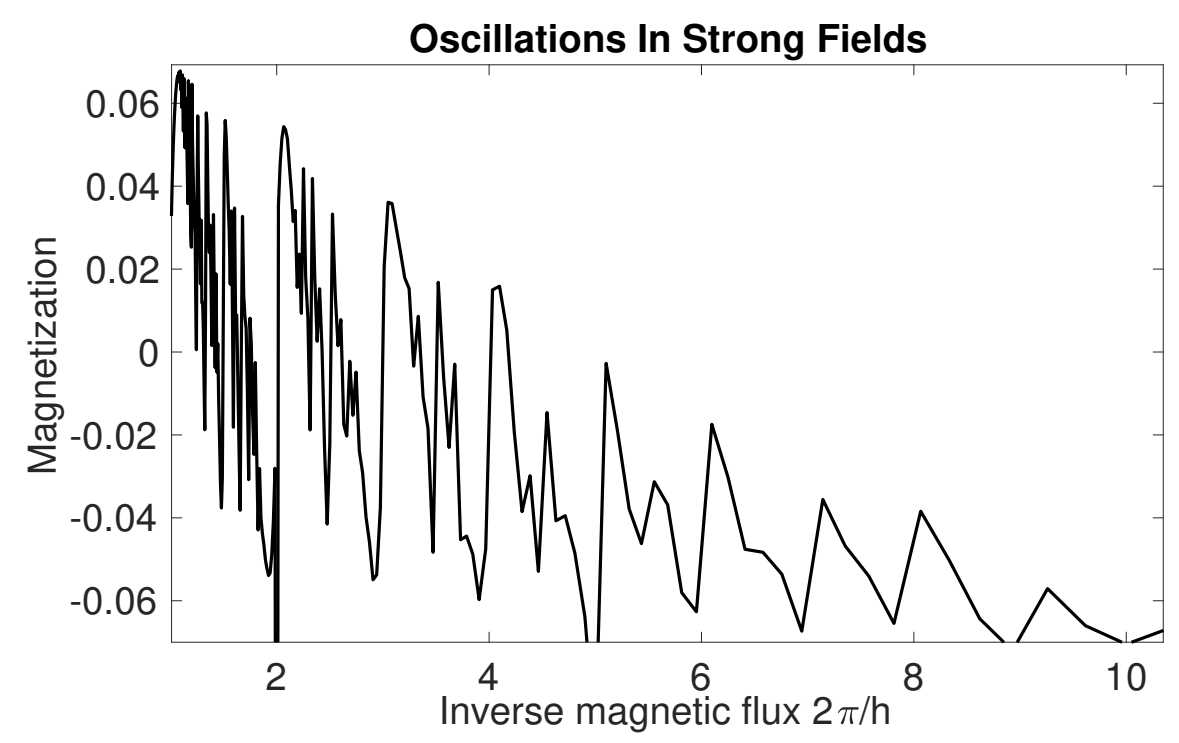

Figure 11. The magnetization (7.9) for a Hamiltonian with zero Hill potential at $\mu=\frac{\pi^{2}}{4}$ given by the Dirac energy. The magnetization shows a decaying inverted saw-tooth profile with oscillations in $1 / h$ and additional high-frequency modulations. As $1 / h$ increases we move to the semiclassical regime in which no oscillations occur at the Dirac energy see Figure 12.

Magnetization is defined by (7.9) and we compute it numerically for (7.24) using finite difference approximation at rational points. Results for computation using difference quotients for magnetic fluxes $h=2 \pi \frac{p}{150}$ and $p \in\{1, \ldots, 150\}$ are shown in Figure 11. The results we obtain are in good agreement with the oscillations obtained in [KH14]. The magnetization shows a decaying inverted saw-tooth profile with oscillations in $1 / h$ and additional high-frequency modulations. These type of magnetic oscillations are an effect of strong magnetic fields. Unlike the dHvA oscillations discussed in $\S 7.2$, the magnetization for such strong magnetic fields deviates significantly from the semiclassical approximation. In particular, the characteristic oscillatory profile caused by the strong magnetic field decreases for sufficiently small magnetic fluxes as we see in Figure 11. Moreover, there are no oscillations when the chemical potential agrees with the energy of the Dirac point in the semiclassical limit.

Figure 12 shows the magnetization (7.9) computed using (7.24) for values of $h$ in the semiclassical regime.

7.4. Comparing spectral and semiclassical calculations. We now compare the exact spectral calculations at magnetic fluxes of the form $h=2 \pi p / q$ (7.23) with the 


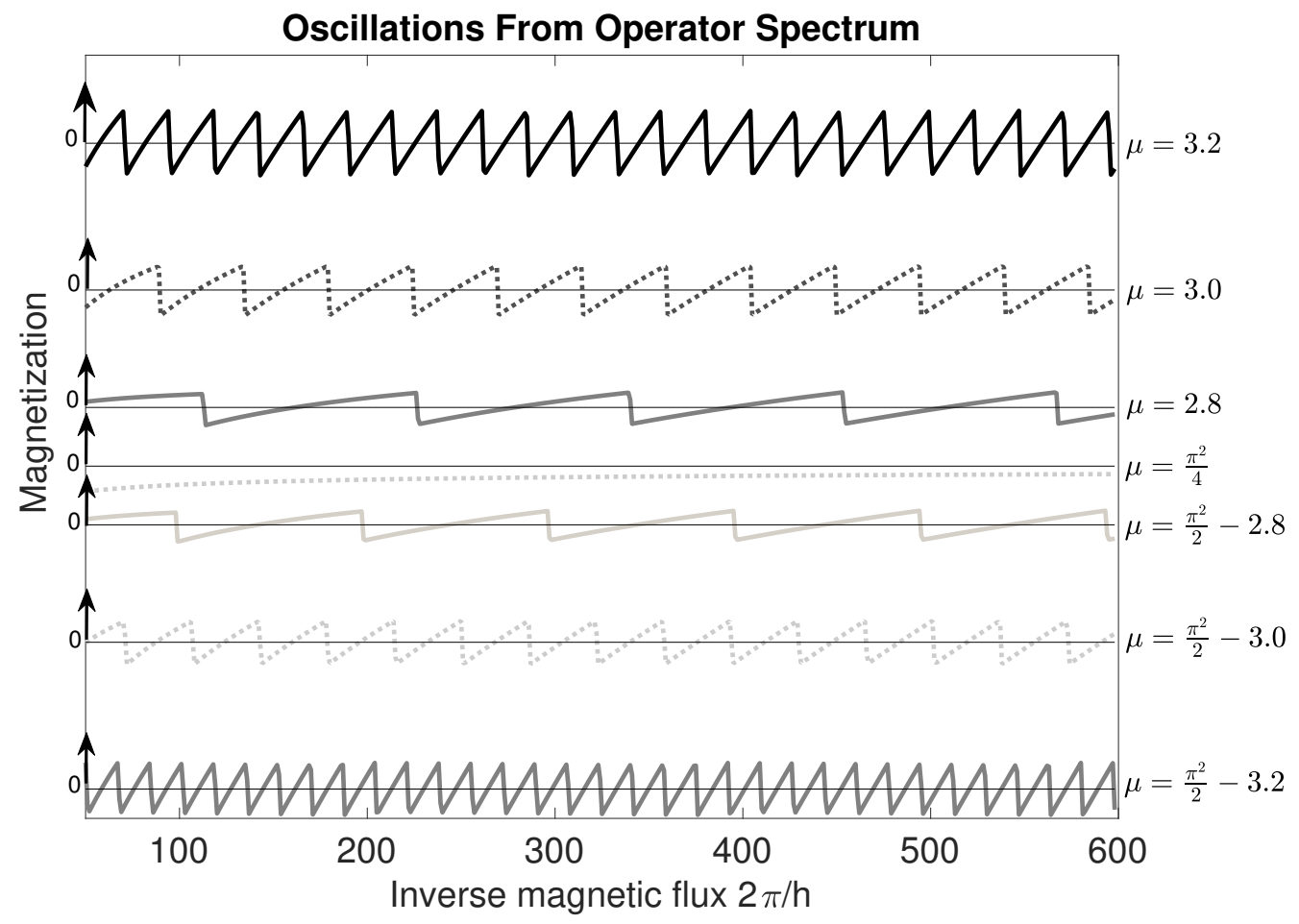

FiguRE 12. The magnetization (7.9) as a function of the inverse flux for specific chemical potentials ( $\mu=\frac{\pi^{2}}{4}$ is the location of the Dirac point) for a magnetic Hamiltonian with zero potential at zero temperature. It is computed using the spectral method (7.24). The magnetization for all chemical potentials is true to scale and calculated at zero temperature from the full operator spectrum (i.e. no cut-off is used). We calculated the magnetization for inverse fluxes $\frac{2 \pi}{q}$ with $q \in\{10, . ., 600\}$. One clearly sees the antisymmetry between the different magnetic oscillations with respect to the conical point. The figures show (away from the Dirac point) jump discontinuities caused by the crossing of chemical potential and Landau levels.

results obtained from the semiclassical trace formula (7.10) where we approximate $z_{h}(n)$ in $(7.7)$ by $z_{h}^{(1)}(n):=g^{-1}(n h)$.

As explained in $\S 7.3$, the spectrum of $H^{B}$ away from the Dirichlet spectrum of $H^{D}$ is fully determined by the eigenvalues of $T_{q}$ as in (7.19). This matrix has for every quasi-momentum $k \in \mathbb{T}_{*}^{2}$ precisely $2 q$ eigenvalues $\lambda_{1}(k) \leq \ldots \leq \lambda_{2 q}(k)$, of which, when pulled back under $\left.\Delta\right|_{B_{k}}$, precisely half are located below and above the conical point $\left.\Delta\right|_{B_{k}} ^{-1}(0)$. Moreover, it is easy to see that there are always two touching bands at the conical point as discussed in [BHJ17] and [HKL16]. 

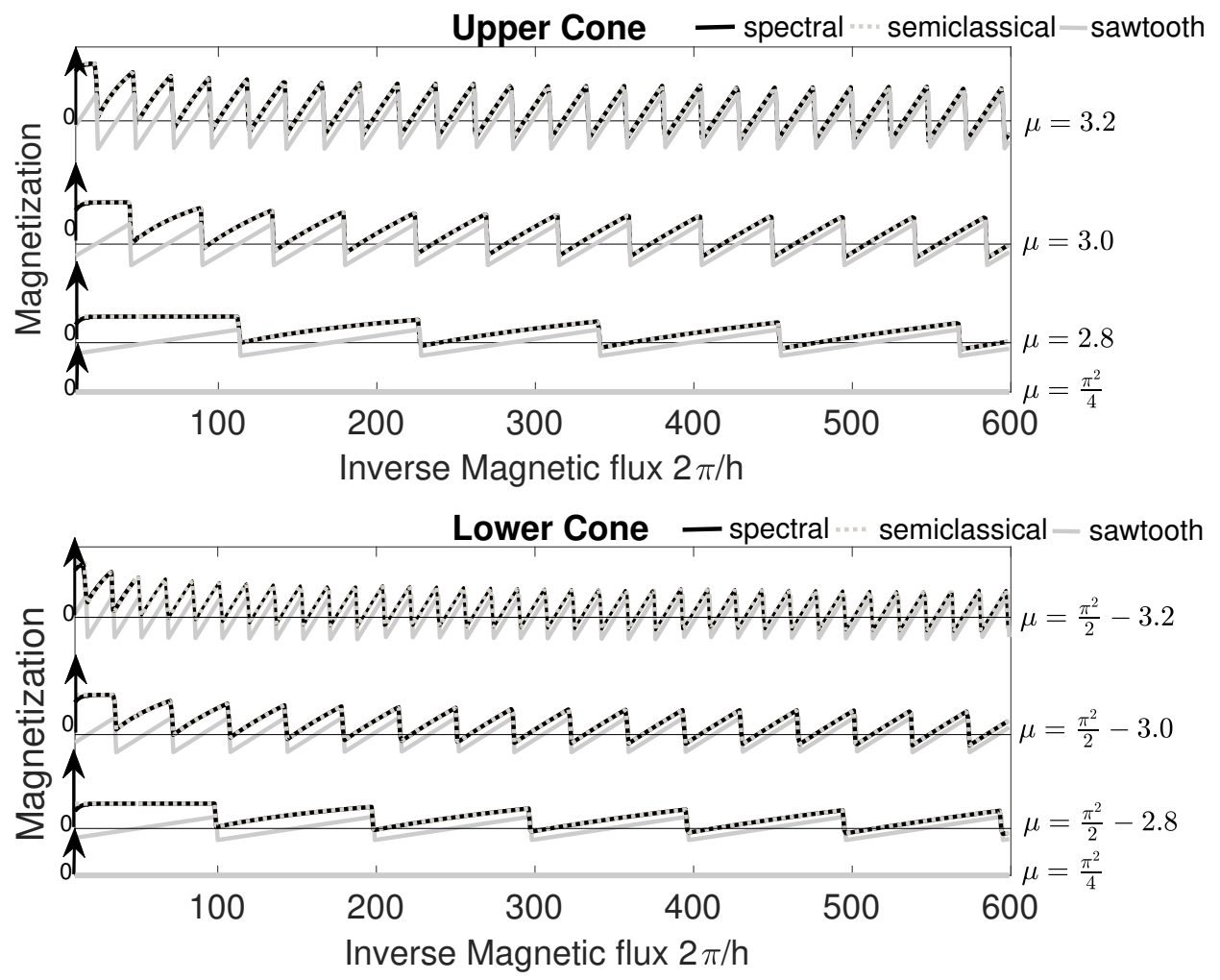

Figure 13. The magnetization for four different chemical potentials above/below the Dirac point located at $\mu=\frac{\pi^{2}}{4}$ on the first Hill band of the magnetic Hamiltonian with zero potential at zero temperature. Continuous lines are computed from the operator spectrum precisely by numerically differentiating (7.26) and dotted lines (grey) from numerically differentiating the semiclassical expression (7.12). We evaluated those expressions for steps $\frac{2 \pi}{q}$ with $q \in\{10, . ., 600\}$. The magnetization for all chemical potentials is true to scale. Both the spectral and semiclassical oscillations show (away from the Dirac point) equally spaced jump discontinuities caused by the crossing of chemical potential and Landau levels. We see that both oscillations coincide up to large magnetic fields (small values of $1 / h$ ).

For chemical potentials $\mu \in\left[z_{D},\left.\Delta\right|_{B_{1}} ^{-1}\left(-\frac{1}{3}\right)\right]$ on the upper cone of the first Hill band we define the grand-canonical potential calculated from DOS of the operator spectrum as in Lemma 7.1

$$
\Omega_{\infty}(\mu, h)=\left(f_{\infty} * \eta \rho_{B}\right)(\mu)=-\frac{1}{q\left|b_{1} \wedge b_{2}\right|} \int_{\mathbb{T}_{*}^{2}} \sum_{i \in\{1, \cdots q\}}\left(\mu-\left.\Delta\right|_{B_{1}} ^{-1}\left(\lambda_{i}(k)\right)\right)_{+} \frac{d k}{\left|\mathbb{T}_{*}^{2}\right|} .
$$


This is the grand-canonical potential calculated from the operator spectrum which corresponds to the semiclassical potential (7.6) with $\beta=\infty$ and $\eta=\Theta_{\frac{1}{2}}$ from (7.11).

For $\mu \in\left[\left.\Delta\right|_{B_{1}} ^{-1}\left(\frac{1}{3}\right), z_{D}\right]$ on the lower cone of the first Hill band, we proceed similarly: in this case, the grand-conical potential, which is defined using the spectrum located between the chemical potential and the conical point, reads

$$
\Omega_{\infty}(\mu, h)=\frac{1}{q\left|b_{1} \wedge b_{2}\right|} \int_{\mathbb{T}_{*}^{2}} \sum_{i \in\{q+1, \cdots 2 q\}}\left(\mu-\left.\Delta\right|_{B_{1}} ^{-1}\left(\lambda_{i}(k)\right)\right)_{-} \frac{d k}{\left|\mathbb{T}_{*}^{2}\right|} .
$$

This potential is the spectral analogue of the semiclassical potential (7.12).

We compare the computation of magnetization (7.9) calculated using finite difference method from (7.25) and (7.26) with the formal semiclassical magnetizations from (7.10) and (7.12) on both cones. The results are shown in Figure 13 and we see a remarkable agreement of the semiclassical approximation with the spectral computation. The sawtooth approximation given in Theorem 3 is also shown.

\section{REFERENCES}

[BHJ17] S. Becker, R. Han, and S. Jitomirskaya, Cantor spectrum in graphene, preprint, 2017.

[BGP07] J. Brüning, V Geyler, and K. Pankrashkin, Cantor and band spectra for periodic quantum graphs with magnetic fields, Communications in mathematical physics, 269(1), 87-105, 2007.

[CU08] P. Carmier and D. Ullmo, Berry phase in graphene: a semiclassical perspective, Phys. Rev. B 77, 245413, 2008.

[CM01] T. Champelde and VP. Mineev, The de Haas-van Alphen effect in two-and quasi-twodimensional metals and superconductors, Philosophical Magazine B, 81, 55-74, 2001.

[CdV80] Y. Colin de Verdière, Spectre conjoint d'opérateurs pseudo-différentiels qui commutent. II. Le cas intégrable, Math. Z. 171, 51-73, 1980.

[DS99] M. Dimassi and J. Sjöstrand, Spectral Asymptotics in the Semi-Classical Limit, Cambridge University Press, 1999.

[DyZw2] S. Dyatlov and M. Zworski, Mathematical theory of scattering resonances, book in preparation; http://math.mit.edu/ dyatlov/res/

[FW12] C. Fefferman and M. Weinstein, Honeycomb lattice potentials and Dirac points, J. Amer. Math. Soc. 25, 1169-1220, 2012.

[G*12] K.K. Gomes, W. Mar, W. Ko, F. Guinea and H.C. Manoharan, Designer Dirac fermions and topological phases in molecular graphene, Nature 483, 306-310, 2012.

[GA03] O. Gat and J.E. Avron. Semiclassical analysis and the magnetization of the Hofstadter model, Phys. Rev. Lett., 91, Issue 18, 2003.

[GS05] V. Gusynin, and S. Sharapov. Magnetic oscillations in planar systems with the Dirac-like spectrum of quasiparticle excitations. II. Transport properties, Phys. Rev. B 71,125124, 2005.

[GS06] V. Gusynin, and S. Sharapov. Transport of Dirac quasiparticles in graphene: Hall and optical conductivities, Physical Review B 73, 245411, 2006.

[HKL16] B. Helffer, P. Kerdelhué, and J. Royo-Letelier. Chambers's formula for the graphene and the Hou model with Kagome periodicity and applications, Annales Henri Poincaré, 17, Issue 4, 2016.

[Ho03] L. Hörmander, The Analysis of Linear Partial Differential Operators I, Springer, 2003. 
[HR84] B. Helffer and D. Robert, Puits de potentiel généralisés et asymptotique semi-classique, Ann. Inst. H. Poincaré Phys. Théor. 41, 291-331, 1984.

[HS88] B. Helffer and J. Sjöstrand, Analyse semi-classique pour l'équation de Harper (avec application à l'équation de Schrödinger avec champ magntique) Mém. Soc. Math. France (N.S.) 34, 1989.

[HS89] B. Helffer and J. Sjöstrand, Equation de Schrödinger avec champ magnétique et équation de Harper. in Schrödinger operators (Sønderborg, 1988), 118-197 Lecture Notes in Phys.345, Springer, Berlin, 1989.

[HS90a] B. Helffer and J. Sjöstrand, Analyse semi-classique pour l'équation de Harper. II. Comportement semi-classique près d'un rationnel. Mém. Soc. Math. France (N.S.) 40, 1990.

[HS90b] B. Helffer and J. Sjöstrand, On diamagnetism and de Haas-van Alphen effect. Ann. Inst. H. Poincaré Phys. Théor. 52, 303-375, 1990.

[KF17] C. Küppersbusch and L. Fritz, Modifications of the Lifshitz-Kosevich formula in twodimensional Dirac systems Phys. Rev. B,96, 205410, 2017.

[KH14] K. Kishigi, Y. Hasegawa, Quantum oscillations of magnetization in tight-binding electrons on a honeycomb lattice, Physical Review B, 90, 085427, 2014.

[KP07] P. Kuchment and O. Post, On the spectra of carbon nano-structures, Communications in Mathematical Physics, 275(3), 805-82, 2007.

[L11] A. Luk'yanchuka, De Haas-van Alphen effect in 2D systems: application to mono- and bilayer graphene, Low Temperature Physics, 37, 45, 2011.

[O52] L. Onsager, Interpretation of the de Haas-van Alphen effect, Philosophical Magazine, 7, 43, 1952.

[Pa06] K. Pankrashkin Spectra of Schrödinger operators on equilateral quantum graphs, Letters in Mathematical Physics, 77(2), 139-154, 2006.

[P*13] M. Polini, F. Guinea, M. Lewenstein, H.C. Manoharan and V. Pellegrini, Artificial honeycomb lattices for electrons, atoms and photons, Nature Nanotechnology 8(2013), 625-633.

[RS78] M. Reed and B. Simon, Analysis of Operators, Vol. IV of Methods of Modern Mathematical Physics, Elsevier, 1978.

[S84] D. Shoenberg, Magnetic Oscillations in Metals, Cambridge University Press, 1984.

[Sch12] K. Schmüdgen, Unbounded Self-adjoint Operators on Hilbert Space, Graduate Texts in Mathematics, Springer, 2012.

[SGB04] S.G. Sharapov, V.P Gusynin and H. Beck, Magnetic oscillations in planar systems with the Dirac-like spectrum of quasiparticle excitations. Phys. Rev. B.69, 075104, 2004.

[Sj89] J. Sjöstrand, Microlocal analysis for periodic magnetic Schrödinger equation and related questions, in Microlocal Analysis and Applications, J.-M. Bony, G. Grubb, L. Hörmander, H. Komatsu and J. Sjöstrand eds. Lecture Notes in Mathematics 1495, Springer, 1989.

[SZ07] J. Sjöstrand and M. Zworski, Elementary linear algebra for advanced spectral problems, Ann. Inst. Fourier 57, 2095-2141, 2007.

[S17] T. Stauber, P. Parida, M. Trushin, M.V. Ulybyshev, D.L. Boyda, and J. Schliemann, Interacting Electrons in Graphene: Fermi Velocity Renormalization and Optical Response, Phys. Rev. Lett. 118, 266801, 2017.

[Tan11] Z. Tan, C. Tan, L. Ma, G. Liu, L. Lu, and C. Yang, Shubnikov-de Haas oscillations of a single layer graphene under dc current bias, Phys. Rev. B 84, 115429, 2011.

[W11] D. Waldmann, et al. Bottom-gated epitaxial graphene, Nature Materials, 10, 357-360, 2011.

[We77] A. Weinstein, Asymptotics of the eigenvalues clusters for the laplacian plus a potential, Duke Math. J. 44, 883-892, 1977.

[Zw12] M. Zworski, Semiclassical analysis, Graduate Studies in Mathematics 138 AMS, 2012. 
E-mail address: simon.becker@damtp.cam.ac.uk

DAmTP, University of Cambridge, Wilberforce Rd, Cambridge CB3 0WA, UK

E-mail address: zworski@math.berkeley.edu

Department of Mathematics, University of California, Berkeley, CA 94720, USA 